\title{
The chemistry of disks around T Tauri and Herbig Ae/Be stars
}

\author{
Marcelino Agúndez ${ }^{1}$, Evelyne Roueff ${ }^{2}$, Franck Le Petit ${ }^{2}$, and Jacques Le Bourlot ${ }^{2,3}$ \\ ${ }^{1}$ Instituto de Física Fundamental, CSIC, C/ Serrano 123, 28006 Madrid, Spain \\ e-mail: marcelino.agundez@csic.es \\ 2 Sorbonne Université, Observatoire de Paris, Université PSL, CNRS, LERMA, 92190 Meudon, France \\ ${ }^{3}$ Université Paris-Diderot, Sorbonne Paris-Cité, 75013 Paris, France
}

Received 21 December 2017 / Accepted 25 March 2018

\begin{abstract}
Context. Infrared and (sub-)millimeter observations of disks around T Tauri and Herbig Ae/Be stars point to a chemical differentiation, with a lower detection rate of molecules in disks around hotter stars.

Aims. We aim to investigate the underlying causes of the chemical differentiation indicated by observations and perform a comparative study of the chemistry of T Tauri and Herbig Ae/Be disks. This is one of the first studies to compare the chemistry in the outer regions of these two types of disk.

Methods. We developed a model to compute the chemical composition of a generic protoplanetary disk, with particular attention to the photochemistry, and applied it to a T Tauri and a Herbig Ae/Be disk. We compiled cross sections and computed photodissociation and photoionization rates at each location in the disk by solving the far-ultraviolet (FUV) radiative transfer in a 1+1D approach using the Meudon PDR code and adopting observed stellar spectra.

Results. The warmer disk temperatures and higher ultraviolet flux of Herbig stars compared to T Tauri stars induce some differences in the disk chemistry. In the hot inner regions, $\mathrm{H}_{2} \mathrm{O}$, and simple organic molecules like $\mathrm{C}_{2} \mathrm{H}_{2}, \mathrm{HCN}$, and $\mathrm{CH}_{4}$ are predicted to be very abundant in T Tauri disks and even more in Herbig Ae/Be disks, in contrast with infrared observations that find a much lower detection rate of water and simple organics toward disks around hotter stars. In the outer regions, the model indicates that the molecules typically observed in disks, like $\mathrm{HCN}, \mathrm{CN}, \mathrm{C}_{2} \mathrm{H}, \mathrm{H}_{2} \mathrm{CO}, \mathrm{CS}, \mathrm{SO}$, and $\mathrm{HCO}^{+}$, do not have drastic abundance differences between T Tauri and Herbig Ae disks. Some species produced under the action of photochemistry, like $\mathrm{C}_{2} \mathrm{H}$ and $\mathrm{CN}$, are predicted to have slightly lower abundances around Herbig Ae stars due to a narrowing of the photochemically active layer. Observations indeed suggest that these radicals are somewhat less abundant in Herbig Ae disks, although in any case, the inferred abundance differences are small, of a factor of a few at most. A clear chemical differentiation between both types of disks concerns ices. Owing to the warmer temperatures of Herbig Ae disks, one expects snow lines lying farther away from the star and a lower mass of ices compared to T Tauri disks.

Conclusions. The global chemical behavior of T Tauri and Herbig Ae/Be disks is quite similar. The main differences are driven by the warmer temperatures of the latter, which result in a larger reservoir or water and simple organics in the inner regions and a lower mass of ices in the outer disk.
\end{abstract}

Key words. astrochemistry - molecular processes - protoplanetary disks - stars: variables: T Tauri, Herbig Ae/Be

\section{Introduction}

Circumstellar disks around young stars, the so-called protoplanetary disks, are an important link in the evolution from molecular clouds to planetary systems. These disks allow feeding of the young star with matter and provide the scenario in which planets form. The study of the physical and chemical conditions of these objects is thus of paramount importance to understanding how planets form and the types of material incorporated. Protoplanetary disks are mainly composed of molecular gas and dust. The last two decades have seen significant progress in the study of their chemical composition thanks to astronomical observations at wavelengths from the millimeter to the ultraviolet (UV) domains.

Observations with ground-based millimeter and submillimeter telescopes such as the $30 \mathrm{~m}$ antenna of the Institut de Radioastronomie Millimétrique (IRAM), the James Clerk Maxwell Telescope (JCMT), and the APEX $12 \mathrm{~m}$ telescope, which are sensitive to the outer cool regions of disks, have provided information on the presence of various gaseous molecules such as $\mathrm{CO}, \mathrm{HCO}^{+}, \mathrm{H}_{2} \mathrm{CO}, \mathrm{C}_{2} \mathrm{H}, \mathrm{HCN}, \mathrm{HNC}, \mathrm{CN}, \mathrm{CS}$, and $\mathrm{SO}$
(Dutrey et al. 1997; Kastner et al. 1997, 2014; van Zadelhoff et al. 2001; Thi et al. 2004; Fuente et al. 2010; Guilloteau et al. 2013, 2016; Pacheco-Vázquez et al. 2015). Interferometers that operate at (sub-)millimeter wavelengths such as the OVRO millimeter array, the IRAM array at Plateau de Bure (PdBI, now known as NOEMA), and the Submillimeter Array (SMA) have also permitted sensitive observations leading to the detection of new molecules such as $\mathrm{N}_{2} \mathrm{H}^{+}$and $\mathrm{HC}_{3} \mathrm{~N}$, and to obtain maps of the emission distribution of some molecules with angular resolutions down to a few arcseconds (Qi et al. 2003, 2008, 2013a; Dutrey et al. 2007, 2011; Piétu et al. 2007; Schreyer et al. 2008; Henning et al. 2010; Öberg et al. 2010, 2011; Chapillon et al. 2012a,b; Fuente et al. 2012; Graninger et al. 2015; Teague et al. 2015; Pacheco-Vázquez et al. 2016). In recent years, the advent of the Atacama Large Millimeter Array (ALMA) has made it possible to characterize the molecular content of protoplanetary disks with unprecedented sensitivity and angular resolution (down to sub-arcsecond scales). For example, thanks to ALMA, it has been possible to detect new molecules such as cyclic $\mathrm{C}_{3} \mathrm{H}_{2}$ (Qi et al. 2013b; Bergin et al. 2016), $\mathrm{CH}_{3} \mathrm{CN}$ (Öberg et al. 2015), and $\mathrm{CH}_{3} \mathrm{OH}$ (Walsh et al. 2016), and to image the $\mathrm{CO}$ snowline 
in a few disks (Mathews et al. 2013; Qi et al. 2013c, 2015; Schwarz et al. 2016; Zhang et al. 2017). Infrared (IR) observations using space telescopes such as Spitzer and ground-based facilities, such as the Very Large Telescope (VLT) and the Keck Observatory telescopes have provided a view of the molecular content of the very inner regions of protoplanetary disks, where absorption and emission lines from molecules, such as $\mathrm{CO}, \mathrm{CO}_{2}, \mathrm{H}_{2} \mathrm{O}, \mathrm{OH}, \mathrm{HCN}, \mathrm{C}_{2} \mathrm{H}_{2}$, and $\mathrm{CH}_{4}$ have been routinely observed (Lahuis et al. 2006; Gibb et al. 2007; Salyk et al. 2007 , 2008, 2011; Carr \& Najita 2008, 2011, 2014; Pontoppidan et al. 2010a,b; Najita et al. 2010, 2013; Kruger et al. 2011; Doppmann et al. 2011; Fedele et al. 2011; Mandell et al. 2012; Bast et al. 2013; Gibb \& Horne 2013; Sargent et al. 2014; Banzatti et al. 2017). The launch of the Herschel Space Observatory was also very helpful to investigate the chemical content at far-IR wavelengths, with the detection of molecules, such as $\mathrm{CH}^{+}$(Thi et al. 2011) and $\mathrm{NH}_{3}$ (Salinas et al. 2016), and the exhaustive characterization of $\mathrm{H}_{2} \mathrm{O}$ and $\mathrm{OH}$ from the inner to the outer regions (Hogerheijde et al. 2011; Riviere-Marichalar et al. 2012; Meeus et al. 2012; Fedele et al. 2012, 2013; Podio et al. 2013). Probing the molecular gas in the inner regions of disks through the most abundant molecule, $\mathrm{H}_{2}$, has also been possible thanks to observations at UV wavelengths using the Hubble Space Telescope (Ingleby et al. 2009; France et al. 2012).

On the theoretical side, the chemical structure of protoplanetary disks has also been widely studied during the last two decades. Early models focused on the one dimensional radial structure of disks along the midplane (Aikawa et al. 1996, 1997, 1999; Willacy et al. 1998; Aikawa \& Herbst 1999a), although it was later recognized that the chemical composition also presents an important stratification along the vertical direction, with a structure consisting of three main layers: the cold midplane where molecules are mostly condensed as ices on dust grains, a warm upper layer where a rich chemistry takes place, and the uppermost surface layer where photochemistry driven by stellar and interstellar far-ultraviolet (FUV) photons regulates the chemical composition (Aikawa \& Herbst 1999b, 2001; Willacy \& Langer 2000; Aikawa et al. 2002). In recent years there has been an interest in identifying the main processes that affect the abundance and distribution of molecules in protoplanetary disks. A large number of studies have addressed in detail the role of processes, such as the interaction with FUV and X-ray radiation (Willacy \& Langer 2000; Markwick et al. 2002; Bergin et al. 2003; Ilgner \& Nelson 2006a; Agúndez et al. 2008; Walsh et al. 2010, 2012; Aresu et al. 2011; Fogel et al. 2011), the interplay between the thermal and chemical structure (Glassgold et al. 2004; Woitke et al. 2009), turbulent mixing and other transport processes (Ilgner et al. 2004; Semenov et al. 2006, 2011; Ilgner \& Nelson 2006b; Willacy et al. 2006; Aikawa 2007; Heinzeller et al. 2011), and the evolution of dust grains as they grow by coagulation and settle onto the midplane regions (Aikawa \& Nomura 2006; Fogel et al. 2011; Akimkin et al. 2013). Some studies have investigated the impact of using different chemical networks (Semenov et al. 2004; Ilgner \& Nelson 2006c; Kamp et al. 2017) and the sensitivity to uncertainties in the rate constants of chemical reactions (Vasyunin et al. 2008). Various specific aspects of the chemistry of protoplanetary disks have also been addressed in detail, such as deuterium fractionation (Willacy 2007; Willacy \& Woods 2009; Thi et al. 2010; Furuya et al. 2013; Yang et al. 2013; Albertsson et al. 2014), the formation and survival of water vapor (Dominik et al. 2005; Glassgold et al. 2009; Bethell \& Bergin 2009; Du \& Bergin 2014), and the formation of particular species such as benzene
(Woods \& Willacy 2007) and complex organic molecules (Walsh et al. 2014).

Overall, protoplanetary disks are complex systems where many different processes govern the chemical composition: for example, gas phase chemistry, interaction with stellar and interstellar FUV photons, transport processes, adsorption, and desorption from dust grains, chemical reactions on grain surfaces, and grain evolution (see reviews by Bergin et al. 2007; Henning \& Semenov 2013; Dutrey et al. 2014; Pontoppidan et al. 2014).

Disks are commonly found around young low-mass (T Tauri) and intermediate-mass (Herbig $\mathrm{Ae} / \mathrm{Be}$ ) stars, which have quite different masses and effective temperatures, and thus can affect the disk chemical composition differently. For example, Herbig Ae/Be stars have a higher UV flux and disks around them are warmer than around T Tauri stars. Indeed, T Tauri and Herbig Ae disks have been extensively observed from millimeter to IR wavelengths and it has been found that the detection rate of molecules (e.g., $\mathrm{H}_{2} \mathrm{O}, \mathrm{C}_{2} \mathrm{H}_{2}, \mathrm{HCN}, \mathrm{CH}_{4}, \mathrm{CO}_{2}, \mathrm{H}_{2} \mathrm{CO}$, $\mathrm{C}_{2} \mathrm{H}$, and $\mathrm{N}_{2} \mathrm{H}^{+}$) is strikingly lower toward Herbig Ae disks than toward disks around T Tauri stars (Mandell et al. 2008; Schreyer et al. 2008; Pontoppidan et al. 2010a; Öberg et al. 2010, 2011; Fedele et al. 2011, 2012, 2013; Salyk et al. 2011; Riviere-Marichalar et al. 2012; Meeus et al. 2012; Guilloteau et al. 2016; Banzatti et al. 2017). This fact may indicate that there is a marked chemical differentiation between these two types of disk. Most theoretical studies on the chemistry of protoplanetary disks have focused on disks around T Tauri-like stars, and only a few have studied Herbig Ae/Be disks (e.g., Jonkheid et al. 2007). Here we present a comparative study in which we investigate the two-dimensional (2D) distribution of molecules in disks around T Tauri and Herbig Ae/Be stars. In a recent study, Walsh et al. (2015) investigated the differences in the chemical composition between disks around stars of different spectral type, focusing on the inner disk regions. In the present study, we make a thorough investigation of the main chemical differences and similarities between T Tauri and Herbig Ae/Be disks from the inner to the outer disk regions, which to our knowledge has not been investigated in detail. We are particularly concerned with a detailed treatment of the photochemistry and with the impact of the FUV illumination from these two types of stars on the chemical composition of the disk. To this purpose we have implemented the Meudon PDR code in the disk model to compute photodestruction rates at each location in the disk in a 1+1D approach. We adopted FUV stellar spectra coming from observations of representative T Tauri and Herbig Ae/Be stars. In Sect. 2 we present in detail our physical and chemical disk model, with particular emphasis on the photochemistry (further described in Appendices A and B). In Sect. 3 we present the resulting abundance distributions of important families of molecules for our T Tauri and Herbig Ae/Be disk models and compare them with results from observations. In Sect. 4 we analyze the influence on the chemistry of the stellar spectra and the method used to compute photodestruction rates, and we summarize the main conclusions found in this work in Sect. 5.

\section{The disk model}

We consider a passively irradiated disk in steady state around a T Tauri or Herbig Ae/Be star. We solve the thermal and chemical structure of the disk using a procedure which can be summarized as follows. We first solve the dust temperature distribution 
Table 1. Model parameters.

\begin{tabular}{|c|c|}
\hline Parameter & Value/Source \\
\hline \multicolumn{2}{|l|}{ Disk } \\
\hline Disk mass $\left(M_{\text {disk }}\right)$ & $0.01 M_{\odot}$ \\
\hline Inner disk radius $\left(R_{\mathrm{in}}\right)$ & 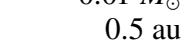 \\
\hline Outer disk radius $\left(R_{\text {out }}\right)$ & $500 \mathrm{au}$ \\
\hline Radial surface density power index $(\epsilon)$ & $1.0 \dagger$ \\
\hline Gas-to-dust mass ratio $\left(\rho_{\mathrm{g}} / \rho_{\mathrm{d}}\right)$ & 100 \\
\hline Dust composition & $\begin{array}{l}70 \% \text { silicate } \\
30 \% \text { graphite }\end{array}$ \\
\hline Minimum dust grain radius $\left(a_{\min }\right)$ & $0.001 \mu \mathrm{m}$ \\
\hline Maximum dust grain radius $\left(a_{\max }\right)$ & $1 \mu \mathrm{m}$ \\
\hline Dust size distribution power index $(\beta)$ & 3.5 \\
\hline Interstellar radiation field & Draine (1978) \\
\hline Cosmic-ray ionization rate of $\mathrm{H}_{2}(\zeta)$ & $5 \times 10^{-17} \mathrm{~s}^{-1}$ \\
\hline \multicolumn{2}{|l|}{ T Tauri star } \\
\hline Stellar mass $\left(M_{*}\right)$ & $0.5 M_{\odot}$ \\
\hline Stellar radius $\left(R_{*}\right)$ & $2 R_{\odot}$ \\
\hline Stellar effective temperature $\left(T_{*}\right)$ & $4000 \mathrm{~K}$ \\
\hline \multicolumn{2}{|l|}{ Herbig Ae/Be star } \\
\hline Stellar mass $\left(M_{*}\right)$ & $2.5 M_{\odot}$ \\
\hline Stellar radius $\left(R_{*}\right)$ & $2.5 R_{\odot}$ \\
\hline Stellar effective temperature $\left(T_{*}\right)$ & $10000 \mathrm{~K}$ \\
\hline
\end{tabular}

Notes. ${ }^{\dagger} \epsilon=-12$ at $r<R_{\text {in }}$.

in the disk using the RADMC code (Dullemond \& Dominik 2004). We assume that gas and dust are thermally coupled except for the surface layers of the disk where we estimate the gas kinetic temperature following Kamp \& Dullemond (2004). We then solve the radiative transfer of interstellar FUV photons along the vertical direction and of stellar FUV photons along the direction from the star using the Meudon PDR code (Le Petit et al. 2006) to get the photodissociation and photoionization rates of the different species at each disk location. We finally solve the chemical composition at each location in the disk as a function of time including gas phase chemical reactions, processes induced by FUV photons and cosmic rays, and interactions of gas particles with dust grains (adsorption and desorption processes).

\subsection{Physical model}

We adopt a fiducial model of disk representative of objects commonly found around T Tauri and Herbig Ae/Be stars (see parameters in Table 1). The disk extends between an inner radius $R_{\text {in }}$ of 0.5 au and an outer radius $R_{\text {out }}$ of 500 au from the star and has a mass $M_{\text {disk }}$ of $0.01 M_{\odot}$. We consider that the radial distribution of the surface density $\Sigma$ is given by a power law of the type

$\Sigma=\Sigma_{0}\left(r / r_{0}\right)^{-\epsilon}$,

where $\Sigma_{0}$ is the surface density at a reference radius $r_{0}$ and the exponent $\epsilon$ is chosen to be 1.0 between $R_{\text {in }}$ and $R_{\text {out }}$. To avoid the abrupt disappearance of the disk at the inner radius we set $\epsilon$ to -12 at $r<R_{\text {in }}$, which results in a soft continuation of the disk at the inner regions.

We consider that dust is present in the disk with a uniform abundance and size distribution, i.e., they do not vary with radius or height over midplane. We adopt a gas-to-dust mass ratio of 100 and consider spherical grains typically present in the interstellar medium, that is, with the composition being a mixture of $70 \%$ of silicate and $30 \%$ of graphite (with optical properties taken from
Draine \& Lee 1984; Laor \& Draine 1993; Weingartner \& Draine 2001), and the size distribution being given by the power law

$n(a) \propto a^{-\beta}$,

where $n(a)$ is the number of grains of radius $a$, the minimum and maximum grain radius $a_{\min }$ and $a_{\max }$ are 0.001 and $1 \mu \mathrm{m}$, and the exponent $\beta$ takes a value of 3.5 according to Mathis et al. (1977).

As stellar parameters we adopt typical values of T Tauri and Herbig Ae/Be stars. The T Tauri star is assumed to have a mass $M_{*}$ of $0.5 M_{\odot}$, a radius $R_{*}$ of $2 R_{\odot}$, and an effective temperature $T_{*}$ of $\sim 4000 \mathrm{~K}$, typical values of T Tauri stars of spectral type M0-K7 in Taurus (Kenyon \& Hartmann 1995). In the case of the star of type Herbig Ae/Be we adopt a mass of $2.5 M_{\odot}$, a radius of $2.5 R_{\odot}$, and an effective temperature of $\sim 10000 \mathrm{~K}$, typical values of early Ae and late Be stars (e.g., Martin-Zaïdi et al. 2008; Montesinos et al. 2009).

\subsubsection{Stellar and interstellar FUV spectra}

The irradiation from the central star is of great importance for the disk because it dominates the heating of gas and dust. The stellar and interstellar spectra at FUV wavelengths are also of prime importance because they control the photochemistry that takes place in the disk surface.

The FUV component of the interstellar radiation field (ISRF) adopted here is given by

$I_{\lambda}=\frac{1}{4 \pi}\left(\frac{6.3622 \times 10^{7}}{\lambda^{4}}-\frac{1.0238 \times 10^{11}}{\lambda^{5}}+\frac{4.0813 \times 10^{13}}{\lambda^{6}}\right)$,

where $\lambda$ is the wavelength in $\AA, I_{\lambda}$ is the specific intensity in units of erg s $\mathrm{cm}^{-1} \AA^{-1} \mathrm{sr}^{-1}$, and the expression has been obtained by fitting to the radiation field given by Draine (1978). The expression in Eq. (3) is similar to that given by Le Petit et al. (2006), except for an erratum in their first term. The ISRF given by Eq. (3) is complemented with another component that accounts for the emission at longer wavelengths (from $\sim 2000 \AA$ to the near IR), for which we adopt the radiation field given by Mathis et al. (1983) in the form of a combination of three diluted black bodies

$$
\begin{aligned}
I_{\lambda}= & 1.05 \times 10^{-14} B_{\lambda}(7127 \mathrm{~K})+1.28 \times 10^{-13} B_{\lambda}(4043 \mathrm{~K}) \\
& +3.30 \times 10^{-13} B_{\lambda}(2930 \mathrm{~K})
\end{aligned}
$$

where $B_{\lambda}(T)$ is the Planck law at temperature $T$.

Concerning stellar spectra, in the case of the T Tauri star we adopt as a proxy of the stellar spectrum that of TW Hya, which consists of observations with the Far Ultraviolet Spectroscopic Explorer (FUSE) in the 900-1150 $\AA$ wavelength range (data from program C0670102) and Hubble STIS observations in the 1150-3150 $\AA$ range (Herczeg et al. 2002; Bergin et al. 2003). The intrinsic stellar brightness is calculated adopting a distance to the star of $51 \mathrm{pc}$ (Mamajek 2005), a stellar radius of $1 R_{\odot}$ (Webb et al. 1999), and a negligible interstellar reddening (Bergin et al. 2003). At wavelengths longer than $3150 \AA$ we use a Kurucz model spectrum (Castelli \& Kurucz 2004) ${ }^{1}$ with an effective temperature of $4000 \mathrm{~K}$, a surface gravity of $10^{1.5} \mathrm{~cm} \mathrm{~s}^{-2}$, and solar metallicity, scaled to match the flux of TW Hya around $3150 \AA$. The resulting spectrum is similar to that presented by France et al. (2014) in the 1150-1750 ̊ wavelength

1 http://wwwuser.oats.inaf.it/castelli 


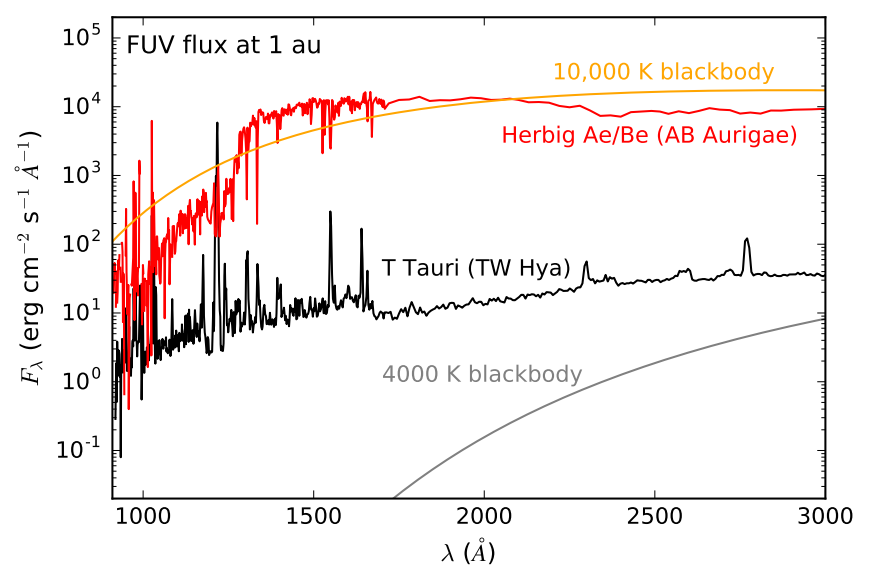

Fig. 1. Stellar FUV flux at 1 au of the T Tauri star (emitting as TW Hya and as a $4000 \mathrm{~K}$ blackbody) and of the Herbig Ae/Be star (emitting as $\mathrm{AB}$ Aurigae and as a $10000 \mathrm{~K}$ blackbody).

range, although their continuum level is about twice below our adopted spectrum. In the case of the star of type Herbig Ae/Be we use as a proxy the FUV spectrum of $\mathrm{AB}$ Aurigae, which consists of observations taken with FUSE in the 900$1190 \AA$ wavelength range (data from program P2190301) and with Hubble STIS in the 1190-1710 A range (Roberge et al. 2001; Ayres 2010). The intrinsic brightness of AB Aurigae is calculated adopting a distance to the star of $144 \mathrm{pc}$, a stellar radius of $2.41 R_{\odot}$, and an interstellar extinction of $0.48 \mathrm{mag}$ (Martin-Zaïdi et al. 2008). For the correction due to extinction we use the method and coefficients of Fitzpatrick \& Massa (2007). Longwards of $1710 \AA$ we use a Kurucz spectrum (Castelli \& Kurucz 2004) with an effective temperature of $9750 \mathrm{~K}$ (close to that of AB Aurigae), a surface gravity of $10^{2.0} \mathrm{~cm} \mathrm{~s}^{-2}$, and solar metallicity.

The FUV spectra adopted for the T Tauri and Herbig Ae/Be stars are shown in Fig. 1, where we also compare with spectra corresponding to blackbodies at the temperatures of the T Tauri and Herbig Ae/Be stars, 4000 and $10000 \mathrm{~K}$, respectively. It is seen that AB Aurigae outshines the FUV flux of TW Hya by 2-3 orders of magnitude due to the much higher effective temperature. However, T Tauri stars usually have an important FUV excess and may become very bright in lines such as Ly $\alpha$ (at $1215.67 \AA$ ). It is worth noting that TW Hya is brighter than $\mathrm{AB}$ Aurigae in the Ly $\alpha$ line and that in TW Hya the fraction of flux emitted in Ly $\alpha$ is about $30 \%$ of the total flux emitted in the 910-2400 A wavelength range. We also note that while the FUV spectra of AB Aurigae is similar to that of a $10000 \mathrm{~K}$ blackbody, a $4000 \mathrm{~K}$ blackbody is a bad approximation for a T Tauri star as it completely misses the FUV excess. As is discussed in Sect. 4, this has important consequences for the chemistry of the disk.

\subsubsection{Dust and gas temperature}

Given the input parameters characteristic of the star $\left(M_{*}, R_{*}\right.$, and stellar spectrum), the radial distribution of surface density in the disk given by Eq. (1), the dust-to-gas mass ratio, and the dust properties, we solve for the $2 \mathrm{D}$ distribution of the dust temperature in the disk using the RADMC code (Dullemond \& Dominik $2004)^{2}$. RADMC is a 2D Monte Carlo code that solves the dust continuum radiative transfer in circumstellar disks and yields the

\footnotetext{
2 http://www.ita.uni-heidelberg.de/ dullemond/ software/radmc/
}

dust temperature as a function of radius $r$ and height $z$ over the midplane. The vertical distribution of the volume density of particles $n(z)$ is assumed to be given by hydrostatic equilibrium as

$\frac{d n(z)}{n(z)}=-\frac{\mu G M_{*}}{k T_{d}(z)} \frac{z}{\left(z^{2}+r^{2}\right)^{3 / 2}} d z$

where $\mu$ is the mean mass of particles, $G$ the gravitational constant, $k$ the Boltzmann constant, and $T_{d}(z)$ is the vertical distribution of dust temperature. We proceed iteratively to find $n(z)$ at each radius $r$ according to Eq. (5) and consistently with the vertical distribution of dust temperature $T_{d}(z)$ computed at each radius $r$.

We assume that gas and dust are thermally coupled, that is, that gas and dust temperatures are equal, except for the disk surface. Models dealing with the computation of the gas temperature in protoplanetary disks find that the thermal coupling of gas and dust is a good approximation over most of the disk and that this assumption breaks down at the surface layers of the disk, where the visual extinction $A_{V}$ in the vertical outward direction becomes lower than $\sim 1$ (Kamp \& Dullemond 2004; Woitke et al. 2009; Walsh et al. 2010). In these surface layers the gas can be much warmer than dust grains. In order to take this into account we use the following approximation for the gas temperature. We assume that the gas temperature is equal to the dust temperature in those regions where $A_{V}$ in the vertical outward direction is higher than 1. Following the study by Kamp \& Dullemond (2004), we assume that in the uppermost regions of the disk, where $A_{V}<0.01$, the gas temperature is equal to the evaporation temperature of a hydrogen atom, calculated as the temperature at which the most probable speed of particles equals the escape velocity from the disk, that is,

$T_{\text {evap }}=\frac{G M_{*} m_{\mathrm{H}}}{k r}$,

where $m_{\mathrm{H}}$ is the mass of a hydrogen atom. Finally, at regions intermediate between $A_{V}=1$ and $A_{V}=0.01$ we approximate the gas temperature through a linear interpolation with height. In Fig. 2 we show the 2D distributions of the volume density of particles and of the gas and dust temperatures calculated for the disks around the T Tauri and Herbig Ae/Be stars. The flared shape, which is apparent in both disks, is more prominent in the $\mathrm{T}$ Tauri disk owing to the lower gravity of the star. The most significant difference between the physical structure of both disks is that the disk around the Herbig Ae/Be star is significantly warmer than the disk around the T Tauri star because of the higher stellar irradiation of the former.

\subsection{Chemical model}

Once the physical structure of the disk (temperature of gas and dust and volume density of particles) is calculated at steady state, we solve for the temporal evolution of the chemical composition at each location in the disk. Since transport processes are neglected, each location in the disk evolves independently of other disk regions. We solve the chemical composition as a function of time up to $1 \mathrm{Myr}$, which is of the order of the typical ages of protoplanetary disks, for a grid consisting of 50 radii (logarithmically spaced from $R_{\text {in }}$ to $R_{\text {out }}$ ) and 200 heights (generated specifically for each radius to properly sample the different regimes of $A_{V}$ in the vertical direction). The chemical network includes 252 species (97 neutral species, 133 positive ions plus the negative ion $\mathrm{H}^{-}$and free electrons, and 20 ice molecules) involving the elements $\mathrm{H}, \mathrm{He}, \mathrm{C}, \mathrm{N}, \mathrm{O}, \mathrm{S}, \mathrm{Cl}$, and $\mathrm{F}$. 

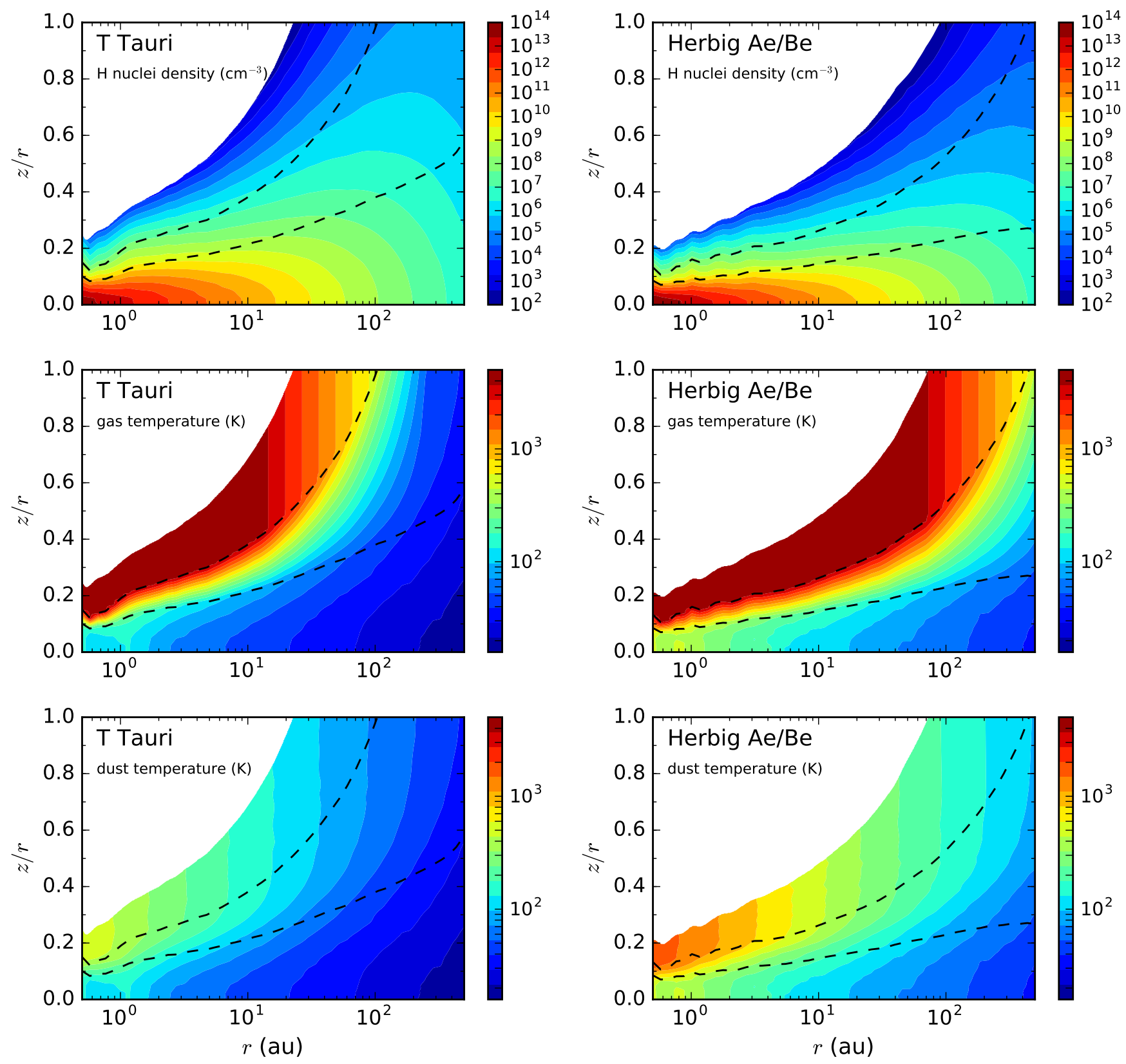

Fig. 2. Calculated volume density of $\mathrm{H}$ nuclei (top panel), gas temperature (middle panel), and dust temperature (bottom panel) as a function of radius $r$ and height over radius $z / r$ for the T Tauri (left panel) and Herbig Ae/Be (right panel) disks. The dashed lines indicate the location where $A_{V}$ in the outward vertical direction takes values of 0.01 and 1.

Atoms of $\mathrm{Si}, \mathrm{P}, \mathrm{Fe}, \mathrm{Na}$, and $\mathrm{Mg}$ are also included because their ionized forms are important in controlling the degree of ionization in certain disk regions. We adopt an initial chemical composition calculated with a pseudo-time-dependent chemical model (where the chemical evolution is solved under fixed physical conditions) of a cold dense cloud with standard parameters (density of $\mathrm{H}$ nuclei of $2 \times 10^{4} \mathrm{~cm}^{-3}$, temperature of $10 \mathrm{~K}$, visual extinction of $10 \mathrm{mag}$, and cosmic-ray ionization rate of $\mathrm{H}_{2}$ of $5 \times 10^{-17} \mathrm{~s}^{-1}$ ) at a time of 0.1 Myr. The elemental abundances adopted, based on the so-called "low metal" case (e.g., Graedel et al. 1982; Lee et al. 1998), are listed in Table 3 of Agúndez \& Wakelam 2013. The chemical network comprises 5533 processes including gas phase chemical reactions, cosmicray induced processes, photodissociations and photoionizations due to stellar and interstellar FUV photons, and exchange processes between the gas and ice mantle phases (adsorption and desorption). At this stage, the model does not include $\mathrm{X}$-ray-induced processes and grain-surface reactions, except for the formation of $\mathrm{H}_{2}$. X-rays may be an important source of chemical differentiation between disks around T Tauri and Herbig Ae/Be stars because the former are more important X-ray emitters (see, e.g., Telleschi et al. 2007). Moreover, winds and magnetic fields in actively accreting $\mathrm{T}$ Tauri systems may lead to cosmic-ray exclusion meaning that ionization in the disk can be dominated by X-rays rather than cosmic rays (Cleeves et al. 2015). Therefore, it will be interesting to study the impact of $\mathrm{X}$-rays on the chemistry of the two types of disks in the future. In any case, previous chemical models of T Tauri disks have found that the gas-phase chemistry is not greatly affected by X-rays. The species whose abundance is most affected are, according to 
Aresu et al. (2011), the ions present in the disk surface $\mathrm{OH}^{+}$, $\mathrm{H}_{2} \mathrm{O}^{+}, \mathrm{H}_{3} \mathrm{O}^{+}$, and $\mathrm{N}^{+}$, while Walsh et al. (2012) find that $\mathrm{N}_{2} \mathrm{H}^{+}$ is the most sensitive species to X-rays. We however note that the models of Aresu et al. (2011) and Walsh et al. (2012) did not consider cosmic-ray exclusion, unlike the study of Cleeves et al. (2015). Chemical reactions occurring on the surface of dust grains are likely to have an effect on the chemical composition of cool midplane regions, especially regarding complex organic molecules (Walsh et al. 2014), although the distribution of abundant molecules and the main chemical patterns in the disk are probably not greatly affected by such processes. We also plan to investigate this particular aspect in the future.

\subsubsection{Gas phase chemical reactions}

The vast majority of gas-phase chemical reactions included can be grouped into two main categories: ion-neutral reactions and neutral-neutral reactions. The subset of ion-neutral reactions has been constructed based on databases originally devoted to the study of the chemistry of cold interstellar clouds, such as the UMIST database for astrochemistry (Woodall et al. 2007; McElroy et al. 2013) ${ }^{3}$ and the Ohio State University (OSU) database, formerly maintained by $\mathrm{E}$. Herbst and currently integrated into the Kinetic Database for Astrochemistry (KIDA; Wakelam et al. $2012,2015)^{4}$. Rate constants of ion-neutral reactions have been taken from the previous databases and from the literature on chemical kinetics. In particular, a large part of the rate constants has been revised according to the compilation by Anicich $(2003)^{5}$. The chemical kinetics of exothermic ion-neutral reactions is rather simple because in most cases the kinetics is dominated by long range electrostatic forces. In the case of reactions in which the neutral species is nonpolar the theory indicates that the rate constant is independent of temperature and is given by the Langevin value. If the neutral species has an electric dipole moment the expression found by Su \& Chesnavich (1982) can be used to evaluate the rate constant and its dependence with temperature (Maergoiz et al. 2009; see more details in Wakelam et al. 2010). For ion-nonpolar reactions for which there is no experimental data, the rate constant has been approximated as the Langevin value. In the case of ion-polar reactions, we have used the Su-Chesnavich approach to evaluate the rate constant of reactions not studied experimentally and to obtain the temperature dependence of the rate constant of reactions which has only been characterized at one single temperature, usually around $300 \mathrm{~K}$.

The part of the chemical network involving ions also includes dissociative recombinations of positive ions with electrons and radiative recombinations between cations and electrons. The set of reactions and associated rate constants have mainly been taken from databases such as UMIST (Woodall et al. 2007; McElroy et al. 2013) and KIDA (Wakelam et al. 2012, 2015). Information on the chemical kinetics of dissociative recombinations has largely benefited from experiments carried out with ion storage rings (Florescu-Mitchell \& Mitchell 2006; Geppert \& Lasson 2008).

The subset of neutral-neutral reactions has been constructed from chemical kinetics databases, such as the one by NIST (Manion et al. 2013) ${ }^{6}$, databases devoted to the study of interstellar chemistry, such as UMIST (Woodall et al. 2007; McElroy

\footnotetext{
3 http://udfa.ajmarkwick.net

4 http://kida.obs.u-bordeaux $1 . f r$

5 http://trs.jpl.nasa.gov/handle/2014/7981

6 http://kinetics.nist.gov
}

et al. 2013) and KIDA (Wakelam et al. 2012, 2015), compilations for application in atmospheric chemistry, such as the evaluations by IUPAC (Atkinson et al. 2004, 2006) ${ }^{7}$ and JPL (Sander et al. 2011) ${ }^{8}$, and compilations for use in combustion chemistry, such as the IUPAC evaluation by Baulch et al. (2005) and the Leeds methane oxidation mechanism (Hughes et al. 2001) or the mechanism by Konnov (2000). A good number of reaction rate constants have been taken from specific experimental and theoretical studies found in the literature on chemical kinetics. For example, we have included the numerous measurements at low and ultra-low temperatures carried out with the CRESU apparatus (Smith et al. 2006). It is important to note that some regions of protoplanetary disks may have temperatures up to some thousands of degrees Kelvin and therefore it is necessary to include reactions that become fast at high temperatures, i.e., reactions which are endothermic and/or have activation barriers. Chemical kinetics data for such reactions are to a large extent based on chemical networks used in previous chemical models of warm gas in protoplanetary nebulae (Cernicharo 2004) and inner regions of protoplanetary disks (Agúndez et al. 2008), whose original sources of data are mainly the combustion chemistry databases listed above. A similar hightemperature chemical network was also used by Harada et al. (2010) to model the chemistry of active galactic nuclei (AGNs). We also include three-body reactions and their reverse process (thermal dissociation) with $\mathrm{H}_{2}, \mathrm{He}$, and $\mathrm{H}$ acting as third body. Three-body reactions become important at densities above $\sim 10^{10} \mathrm{~cm}^{-3}$, values that are reached in the innermost midplane regions of protoplanetary disks, while thermal dissociations become important at high temperatures. We use an expanded version of the set of three-body reactions and thermal dissociations compiled by Agúndez \& Cernicharo (2006). An important aspect of the neutral-neutral subset of reactions is that for many of the endothermic reactions for which chemical kinetics data are not available the rate constants have been calculated through detailed balance from the rate constant of the reverse exothermic reaction and the thermochemical properties of the species involved. Thermochemical data in the form of NASA polynomial coefficients (McBride et al. (2002) have been obtained from compilations like those by Konnov (2000) and Burcat \& Ruscic (2005) 9 .

\subsubsection{Cosmic-ray-induced processes}

The processes induced by cosmic rays also play an important role in the chemistry of protoplanetary disks. We include the direct ionization of $\mathrm{H}_{2}$ and atoms by cosmic-ray impact, together with photoprocesses induced by secondary electrons produced in the direct ionization of $\mathrm{H}_{2}$, the so-called Prasad-Tarafdar mechanism (Prasad \& Tarafdar 1983; Gredel et al. 1989). The rates of these processes are expressed in terms of the cosmic-ray ionization rate of $\mathrm{H}_{2}(\zeta)$, for which we adopt a value of $5 \times 10^{-17} \mathrm{~s}^{-1}$ (see Table 1), and are taken from astrochemical databases such as UMIST (Woodall et al. 2007; McElroy et al. 2013) and KIDA (Wakelam et al. 2012, 2015).

\subsubsection{Photoprocesses}

Photodissociation and photoionization processes caused by stellar and interstellar FUV photons are a key aspect of the chemistry of protoplanetary disks as they control the chemical composition of the surface layers, from where much of the molecular

\footnotetext{
7 http://iupac.pole-ether.fr/

8 http://jpldataeval.jpl.nasa.gov/

9 http://burcat.technion.ac.il/dir
} 
emission arises. In order to treat these processes in detail we have used the Meudon PDR code (Le Petit et al. 2006; Goicoechea \& Le Bourlot 2007; González García et al. 2008) ${ }^{10}$, where PDR stands for photodissociation region, to compute the photodissociation and photoionization rates of various important species at each location in the disk. We use version 1.4 of the Meudon PDR code, with some practical modifications to make it more versatile and integrate it into the protoplanetary disk model. The Meudon PDR code solves the FUV radiative transfer in one dimension for a plane-parallel cloud illuminated on one side by a FUV source. In protoplanetary disks, the geometry involves two dimensions (radial and vertical) and there are two different FUV sources, the star illuminating from the central position and the interstellar radiation field illuminating isotropically from outside the disk. We thus adopt a 1+1D approach. On the one hand we solve the radiative transfer of FUV interstellar radiation as it propagates from outside the disk to the midplane along a series of vertical directions located at the different radii of the grid described in Sect. 2.2. On the other hand we solve the radiative transfer of FUV stellar radiation as it travels from the star through the disk along a series of directions given by a grid of 200 angles from the midplane (covering the range from $0^{\circ}$ to almost $90^{\circ}$ ). The Meudon PDR code assumes that stellar photons arrive from a direction perpendicular to the plane-parallel surface of the cloud. In disks, the star may illuminate the disk with small grazing angles, in particular in the inner regions where the flaring shape of the disk is less marked. It is thus likely that when computing the FUV energy density along the different directions from the star, at low penetration depths the Meudon PDR code underestimates the contribution of FUV photons scattered by dust from nearby regions around the disk surface. However, it is not straightforward to properly correct by this geometrical effect without moving to $2 \mathrm{D}$ and therefore we do not apply any specific correction for it here.

In summary, once the physical structure of the disk is calculated at steady state (using the RADMC code as described in Sect. 2.1) and prior to the computation of the temporal evolution of the chemical composition, we use the Meudon PDR code to evaluate the photodissociation and photoionization rates at each location in the disk by calculating the FUV flux as a function of wavelength due to stellar and interstellar radiation and using the relevant wavelength-dependent cross sections. Our approach to treat photochemistry is thus different from other state-of-theart chemical models of protoplanetary disks in which the FUV radiative transfer is solved in $2 \mathrm{D}$ but only in a few broad spectral bands (e.g., Woitke et al. 2016) and it is in essence more similar to the series of models by Walsh et al. (2012, 2014, 2015).

We have compiled cross sections for 29 molecules and 8 atoms (see Appendix A). The photodissociation rate of $\mathrm{H}_{2}$ and $\mathrm{CO}$ are computed by solving the excitation and the line-by-line radiative transfer taking into account self and mutual shielding effects. In the case of photoprocesses for which cross section data is not available we have approximated the rate using a parametric expression in which the rate $\Gamma$, in units of $\mathrm{s}^{-1}$, is expressed as a function of the visual extinction $A_{V}$ as

$\Gamma=\chi \alpha \exp \left(-\gamma A_{V}\right)$

where $\chi$ is the FUV ${ }^{11}$ energy density with respect to that of the ISRF of Draine (1978), $\alpha$ is the rate under a given unattenuated radiation field with $\chi=1$, and the coefficient $\gamma$ controls the

${ }_{10}$ http://ism.obspm.fr

11 The FUV is chosen to cover the wavelength range from the Lyman cutoff at $911.776 \AA$ to the limit of the Habing field at $2400 \AA$.
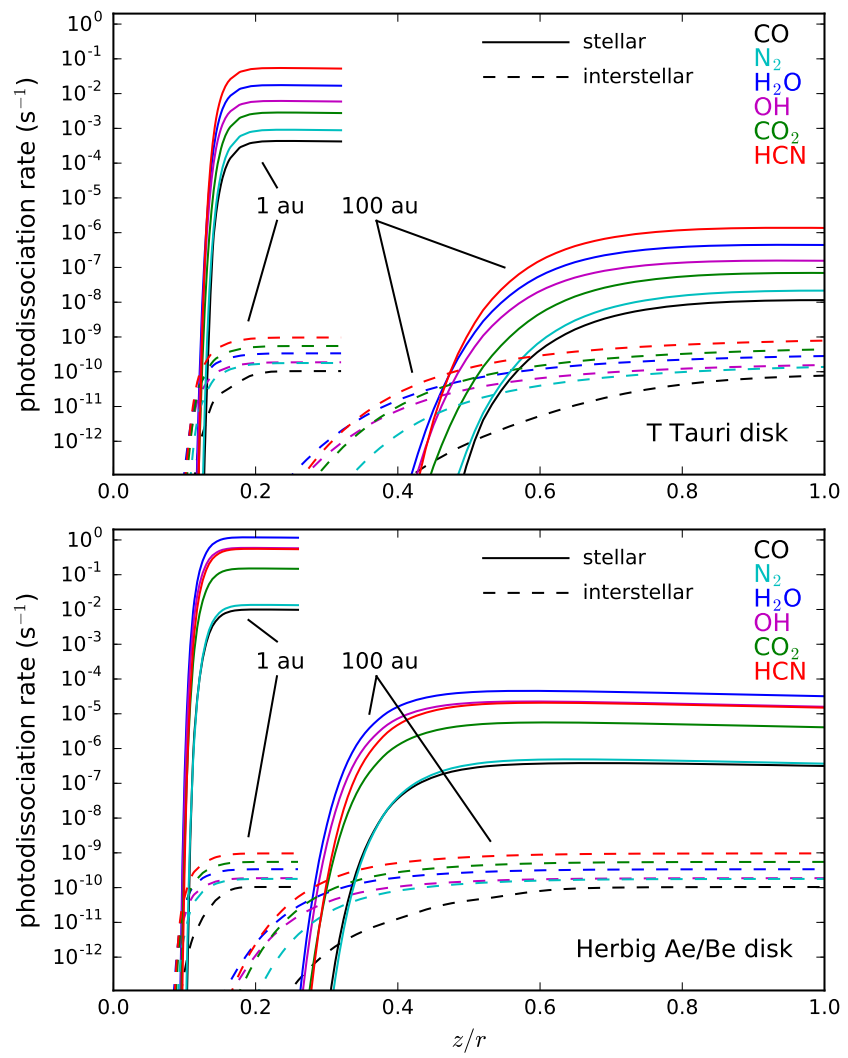

Fig. 3. Contribution of the stellar (solid lines) and interstellar (dashed lines) FUV fields to the photodissociation rates of various molecules as a function of the height over radius $(z / r)$ at $1 \mathrm{au}$ and $100 \mathrm{au}$ in the T Tauri (upper panel) and Herbig Ae/Be (lower panel) disks.

decrease in the rate with increasing visual extinction. The coefficients $\alpha$ and $\gamma$ are specific of each photoprocess and depend also on the spectral shape of the FUV field. Values of $\alpha$ and $\gamma$ corresponding to the ISRF have been taken from the databases such as the UMIST database for astrochemistry (Woodall et al. 2007; McElroy et al. 2013) and the OSU and KIDA databases (Wakelam et al. 2012, 2015), as well as from the compilation by van Dishoeck et al. (2006), recently revised by Heays et al. (2017).

In Fig. 3 we show the contribution of the stellar and interstellar radiation fields to the photodissociation rate of various molecules as a function of height over the disk midplane. This is shown at two radial distances from the star (1 au and $100 \mathrm{au}$ ) for the T Tauri and Herbig Ae/Be disks. We see that in the uppermost regions of the disk, photoprocesses are clearly dominated by the stellar radiation field. However, as we go deeper into the disk, at some point the contribution of the ISRF becomes more important than the stellar one because of the more marked increase of the visual extinction against stellar light than against interstellar photons. Therefore, depending on the location in the disk, photodestruction can be driven by the ISRF or by the star.

\subsubsection{Reactions with vibrationally excited $\mathrm{H}_{2}$}

At the disk surface, the gas is strongly illuminated by FUV photons and vibrationally excited states of molecular hydrogen are easily populated through FUV fluorescence. Since the reactivity of $\mathrm{H}_{2}$ can be quite different when it is in the ground or in excited vibrational states - the internal energy of $\mathrm{H}_{2}$ can be used to overcome or diminish endothermicities or activation barriers which are present when $\mathrm{H}_{2}$ is in its ground vibrational 
state - we have included a few reactions of $\mathrm{H}_{2}$ with specific rate constants for each vibrational state. Specifically, we have included the reactions of $\mathrm{H}_{2}$ with $\mathrm{C}^{+}$(important in the formation of $\left.\mathrm{CH}^{+}\right), \mathrm{He}^{+}, \mathrm{O}, \mathrm{OH}$, and $\mathrm{CN}$, with the rate constant expressions compiled by Agúndez et al. (2010), and the reaction of $\mathrm{H}_{2}$ and $\mathrm{S}^{+}$, which may be important in the synthesis of the ion $\mathrm{SH}^{+}$, with the rate constant expressions calculated by Zanchet et al. (2013). The populations of the different vibrational states of $\mathrm{H}_{2}$ are computed at each location in the disk with the Meudon PDR code.

\subsubsection{Adsorption processes}

The adsorption of gas species onto the surface of dust grains is treated in a rather simple and standard way. The adsorption rate of a gas species $i$, in units of $\mathrm{s}^{-1}$, is given by

$R_{i}^{\text {ads }}=\alpha_{i} v_{i}\left\langle\sigma_{d} n_{d}\right\rangle$,

where $\alpha_{i}$ and $v_{i}$ are the sticking coefficient and thermal velocity of each gas species $i$. The sticking coefficient is assumed to be 1 for all species and the thermal velocity is evaluated as $\sqrt{3 k T_{k} / m_{i}}$, where $T_{k}$ is the gas kinetic temperature and $m_{i}$ is the mass of each gas species $i$. The term $\left\langle\sigma_{d} n_{d}\right\rangle$ is the product of the geometric cross section and the volume density of dust particles averaged over the grain size distribution given by Eq. (2) and is evaluated following the formalism of Le Bourlot et al. (1995). To simplify, we only consider adsorption of a limited number of stable neutral species, among which there are the most typically abundant ice constituents (see Table 2)

\subsubsection{Desorption processes}

Species which have been adsorbed on dust grains forming ice mantles can return to the gas phase through a variety of desorption mechanisms. In protoplanetary disks, the most important desorption processes are thermal desorption and photodesorption by FUV photons. We also include desorption induced by cosmic rays. Based on the results of experimental work using isotopic markers (Bertin et al. 2012, 2013) and molecular dynamics calculations (Andersson \& van Dishoeck 2008), we consider that only molecules from the top two monolayers can desorb efficiently $\left(N_{l}=2\right)$. The term due to desorption can therefore be written in the kinetic rate equations as

$\frac{d n_{i}}{d t}=-\frac{d n_{i}^{\mathrm{ice}}}{d t}=\left(R_{i}^{\mathrm{thd}}+R_{i}^{\mathrm{pd}}+R_{i}^{\mathrm{crd}}\right) n_{i}^{\text {ice, desorbable }}$,

where $n_{i}$ and $n_{i}^{\text {ice }}$ are the volume densities of species $i$ in the gas and ice phases, respectively, $R_{i}^{\text {thd }}, R_{i}^{\mathrm{pd}}, R_{i}^{\text {crd }}$ are the desorption rates, in units of $\mathrm{s}^{-1}$, of thermal desorption, photodesorption, and desorption induced by cosmic rays, respectively (see below). The quantity $n_{i}^{\text {ice,desorbable }}$ is the volume density of species $i$ in the top desorbable ice layers, which is simply equal to $n_{i}^{\text {ice }}$ when these layers are not fully occupied while it is given by $n_{i}^{\text {ice }}$ multiplied by the factor $\left(n_{\text {top }} / n_{\text {tot }}^{\text {ice }}\right)$ otherwise. The volume density of sites in the top desorbable layers $n_{\text {top }}$ is given by $n_{s} 4\left\langle\sigma_{d} n_{d}\right\rangle N_{l}$ (where $n_{s}$ is the surface density of sites, typically $\sim 1.5 \times 10^{15} \mathrm{~cm}^{-2}$; see Hasegawa et al. 1992) and $n_{\mathrm{tot}}^{\text {ice }}$ is the sum of the volume densities of all ice species, that is, $\sum_{i} n_{i}^{\text {ice }}$. This implementation of desorption in the kinetic rate equations is similar to that of, for example, Aikawa et al. (1999) and Woitke et al. (2009); see also Cuppen et al. (2017).
Table 2. Ice species, binding energies, and photodesorption yields.

\begin{tabular}{|c|c|c|c|c|}
\hline Species & $E_{D}(\mathrm{~K})$ & Ref. & $Y\left(\right.$ molecule photon $\left.^{-1}\right)$ & Ref. \\
\hline $\mathrm{CH}_{4}$ & 1000 & $(1)^{\diamond}$ & $10^{-3}$ & (11) \\
\hline $\mathrm{C}_{2} \mathrm{H}_{2}$ & 2587 & $(2)^{\ddagger}$ & $10^{-3}$ & (11) \\
\hline $\mathrm{C}_{2} \mathrm{H}_{4}$ & 3487 & $(2)^{\ddagger}$ & $10^{-3}$ & (11) \\
\hline $\mathrm{C}_{2} \mathrm{H}_{6}$ & 4387 & $(2)^{\ddagger}$ & $10^{-3}$ & (11) \\
\hline $\mathrm{H}_{2} \mathrm{O}$ & 5773 & $(3)^{\diamond}$ & $\left(1.3+0.032 T_{d}\right) \times 10^{-3}$ & $(12) *$ \\
\hline $\mathrm{O}_{2}$ & 1161 & $(4)^{\dagger}$ 七 & $\left(2.8^{a}, 2.3^{b}, 2.4^{c}\right) \times 10^{-3}$ & $(13) \S$ \\
\hline $\mathrm{CO}$ & 1575 & $(5)^{\dagger}$ & $\left(9.5^{a}, 4.8^{b}, 10.3^{c}\right) \times 10^{-3}$ & (14) $\S$ \\
\hline $\mathrm{CO}_{2}$ & 2346 & $(4)^{\dagger}$ & $\left(8.2^{a}, 2.0^{b}, 0.98^{c}\right) \times 10^{-4}$ & $(15) \S *$ \\
\hline $\mathrm{H}_{2} \mathrm{CO}$ & 3260 & $(6)^{\dagger}$ & $10^{-3}$ & (11) \\
\hline $\mathrm{CH}_{3} \mathrm{OH}$ & 4990 & $(7)^{\diamond}$ & $\left(1.5^{a}, 1.1^{b}, 1.3^{c}\right) \times 10^{-4}$ & $(16) \S *$ \\
\hline $\mathrm{HCOOH}$ & 5570 & $(2)^{\ddagger}$ & $10^{-3}$ & (11) \\
\hline $\mathrm{NH}_{3}$ & 3830 & $(1)^{\diamond}$ & $10^{-3}$ & (11) \\
\hline $\mathrm{N}_{2}$ & 1435 & $(5)^{\dagger}$ & $\left(2.0^{a}, 1.6^{b}, 0.89^{c}\right) \times 10^{-3}$ & $(13) \S$ \\
\hline $\mathrm{HCN}$ & 2050 & $(2)^{\ddagger}$ & $10^{-3}$ & (11) \\
\hline $\mathrm{H}_{2} \mathrm{~S}$ & 1945 & $(8)^{\#}$ & $10^{-3}$ & (11) \\
\hline $\mathrm{CS}$ & 1900 & $(2)^{\ddagger}$ & $10^{-3}$ & (11) \\
\hline $\mathrm{H}_{2} \mathrm{CS}$ & 2700 & $(2)^{\ddagger}$ & $10^{-3}$ & (11) \\
\hline SO & 2600 & $(2)^{\ddagger}$ & $10^{-3}$ & (11) \\
\hline $\mathrm{SO}_{2}$ & 3900 & $(9)^{\dagger}$ & $10^{-3}$ & (11) \\
\hline OCS & 3440 & $(10)^{\diamond}$ & $10^{-3}$ & (11) \\
\hline
\end{tabular}

Notes. ${ }^{\diamond}$ Pure ice; ${ }^{\dagger}$ amorphous water ice substrate ( ${ }^{\natural}$ submonolayer regime); ${ }^{\sharp}$ solid $\mathrm{SO}_{2}$ substrate; ${ }^{\ddagger}$ estimated for water ice substrate; $\S$ wavelength-dependent photodesorption yield is convolved over the 7-13.6 eV range with the interstellar ${ }^{(a)}, \mathrm{T} \mathrm{Tauri}^{(b)}$, and Herbig Ae/Be ${ }^{(c)}$ FUV radiation fields described in Sect. 2.1.1; *yields of direct photodesorption and fragmentation have been measured.

References. (1) Luna et al. (2014); (2) Garrod \& Herbst (2006); (3) Fraser et al. (2001); (4) Noble et al. (2012a); (5) Fayolle et al. (2016); (6) Noble et al. (2012b); (7) Doronin et al. (2015); (8) Sandford \& Allamandola (1993); (9) Schriver-Mazzuoli et al. (2003), assuming that $E_{D}$ is directly proportional to the desorption temperature (see, e.g., Martín-Doménech et al. 2014); (10) Burke \& Brown (2010); (11) assumed; (12) pure $\mathrm{H}_{2} \mathrm{O}$ ice (>8 ML) in the $18-100 \mathrm{~K}$ range; the fraction of $\mathrm{H}_{2} \mathrm{O}$ molecules photodesorbed is $\left(0.42+0.002 T_{d}\right)$, the remaining results in fragmentation into $\mathrm{OH}+\mathrm{H}$ (Öberg et al. 2009); (13) pure $\mathrm{O}_{2}$ (30 ML) and ${ }^{15} \mathrm{~N}_{2}$ (60 ML) ices at $15 \mathrm{~K}$ (Fayolle et al. 2013); (14) pure CO ice (10 ML) at $18 \mathrm{~K}$ (Fayolle et al. 2011); (15) pure ${ }^{13} \mathrm{CO}_{2}(10 \mathrm{ML})$ ice at $10 \mathrm{~K}$; the fraction of $\mathrm{CO}_{2}$ molecules desorbed is $0.37,0.42$, and 0.22 under the interstellar, T Tauri, and Herbig Ae/Be FUV fields, respectively, the remaining results in fragmentation into $\mathrm{CO}+\mathrm{O}$ (Fillion et al. 2014); (16) pure $\mathrm{CH}_{3} \mathrm{OH}(20 \mathrm{ML})$ ice at $10 \mathrm{~K}$; the fraction of $\mathrm{CH}_{3} \mathrm{OH}$ molecules desorbed is 0.08 , while the fragmentation channels $\mathrm{CH}_{3}+\mathrm{OH}, \mathrm{H}_{2} \mathrm{CO}+\mathrm{H}_{2}$, $\mathrm{CO}+\mathrm{H}_{2}+\mathrm{H}_{2}$ occur with fractions of $0.05,0.07$, and 0.80 , almost independently of the radiation field (Bertin et al. 2016; see also Cruz-Diaz et al. 2016).

- Thermal desorption. This process, which depends on the dust temperature $T_{d}$ and the binding energy of adsorption of each species $E_{D}$, controls to a large extent the distribution of ices in protoplanetary disks and the location of the different snow lines of each molecule. The thermal desorption rate, in units of $\mathrm{s}^{-1}$, of an adsorbed species $i$ is given by

$R_{i}^{\mathrm{thd}}=v_{0, i} \exp \left(-E_{D, i} / T_{d}\right)$,

where $v_{0, i}$ is the characteristic vibration frequency of the adsorbed species $i$ (evaluated as $\sqrt{2 n_{s} k E_{D, i} /\left(\pi^{2} m_{i}\right)}$, where the binding energy $E_{D, i}$ is expressed in units of $\mathrm{K}$. Binding energies 
have been measured in the laboratory by depositing volatile species on different cold substrates and using temperature programmed desorption methods (see Burke \& Brown 2010 and references therein). In general, binding energies show little dependence on the chemical or morphological nature of the substrate as long as the ice under study consists of various monolayers (ML). If desorption occurs at submonolayer coverage, desorption energies can be quite different depending on the substrate; for example, they tend to be higher when employing water ice as a substrate than when using silicates (Noble et al. 2012a). The translation of these laboratory experiments into a realistic model of thermal desorption in protoplanetary disks is complicated because ices are heterogeneous mixtures, thought to be dominated by water ice but whose composition probably varies between cold and warmer regions. Also, molecules may selectively co-desorb with other trapped species, experience volcano desorption following the crystallization of water ice, or co-desorb with some of the major ice constituents (Collings et al. 2004). Here we have adopted the simple and usual approach in which the thermal desorption of each species is controlled by a specific binding energy. We have collected values of experimentally measured $E_{D}$, when possible using a water ice substrate and under a submonolayer regime. For those molecules for which experimental binding energies are not available, we have adopted the values estimated by Garrod \& Herbst (2006) for a water ice substrate based on the previous compilation by Hasegawa \& Herbst (1993) and the experimental study of Collings et al. (2004). The binding energies of the ice molecules considered and the corresponding references are given in Table 2.

- Photodesorption. The absorption of FUV photons of stellar or interstellar origin (or generated through the Prasad-Tarafdar mechanism) by icy dust grains can induce the desorption of molecules on the ice surface. In regions where the dust temperature is too cold to allow for thermal desorption, photodesorption can provide an efficient means to bring ice molecules to the gas phase. The photodesorption rate, in units of $\mathrm{s}^{-1}$, of an adsorbed species $i$ is given by

$R_{i}^{p d}=Y_{i} F_{\mathrm{FUV}} \frac{\left\langle\sigma_{d} n_{d}\right\rangle}{4\left\langle\sigma_{d} n_{d}\right\rangle n_{s} N_{l}}$,

where $Y_{i}$ is the yield of molecules desorbed per incident photon, $F_{\mathrm{FUV}}$ is the FUV photon flux (in units of photon $\mathrm{cm}^{-2} \mathrm{~s}^{-1}$ ), and the expression of $R_{i}^{p d}$, when inserted in Eq. (9), naturally accounts for the fact that ices are not pure but consist of multiple constituents and that desorption is only effective from the top desorbable layers (see, e.g., Cuppen et al. 2017). FUV photons may have a stellar or interstellar origin (in which case $F_{\text {FUV }}$ is evaluated with the Meudon PDR code at each position in the disk) or can be generated through the Prasad-Tarafdar mechanism, in which case we adopt $F_{\mathrm{FUV}}=2 \times 10^{3}$ photon $\mathrm{cm}^{-2} \mathrm{~s}^{-1}$ (values between 750 and $10^{4}$ photon $\mathrm{cm}^{-2} \mathrm{~s}^{-1}$ have been reported in the literature; e.g., Hartquist \& Williams 1990; Shen et al. 2004). We note that this latter value scales with the cosmic-ray ionization rate. Experiments carried out to study the photodesorption of pure ices or binary ice mixtures (Öberg et al. 2009; Muñoz Caro et al. 2010; Fayolle et al. 2011, 2013; Bertin et al. 2013; Fillion et al. 2014; Martín-Doménech et al. 2015) suggest that the main underlying mechanism, called desorption induced by electronic transition (DIET), involves absorption of FUV photons in approximately the top five monolayers and electronic excitation of the absorbing molecules, followed by energy redistribution to neighboring molecules, which may break their intermolecular bonds and be ejected into the gas phase. The efficiency of photodesorption is thus regulated by the ability of the molecules present in the ice surface and sub-surface to absorb FUV photons through electronic transitions. If these transitions are dissociative, the situation becomes more complex because the fragments may desorb directly, recombine in the ice, and then desorb or diffuse through the ice forming new molecules that may also desorb (e.g., Andersson \& van Dishoeck 2008). Here we adopt a simple approach in which ice molecules may desorb directly or as fragments upon FUV irradiation, with yields based on experimental data for $\mathrm{CO}, \mathrm{N}_{2}$, $\mathrm{O}_{2}, \mathrm{H}_{2} \mathrm{O}, \mathrm{CO}_{2}$, and $\mathrm{CH}_{3} \mathrm{OH}$ (see Table 2). Desorption of fragments has been observed upon irradiation of pure ices of $\mathrm{H}_{2} \mathrm{O}$, $\mathrm{CO}_{2}$, and $\mathrm{CH}_{3} \mathrm{OH}$ (Öberg et al. 2009; Fillion et al. 2014; MartínDoménech et al. 2015; Bertin et al. 2016; Cruz-Diaz et al. 2016), and it is likely that desorption of dissociation fragments and new species formed in situ in the ice dominates over direct desorption for other polyatomic molecules. However, in the absence of experimental photodesorption yields for molecules other than $\mathrm{CO}, \mathrm{N}_{2}, \mathrm{O}_{2}, \mathrm{H}_{2} \mathrm{O}, \mathrm{CO}_{2}$, and $\mathrm{CH}_{3} \mathrm{OH}$ we have assumed that direct desorption dominates with assumed values for $Y_{i}$. It is interesting to note that photodesorption yields of $\mathrm{CO}, \mathrm{O}_{2}, \mathrm{~N}_{2}, \mathrm{CO}_{2}$, and $\mathrm{CH}_{3} \mathrm{OH}$ have been measured as a function of wavelength using synchrotron techniques (Fayolle et al. 2011, 2013; Fillion et al. 2014; Bertin et al. 2016), which permits to compute the yield $Y_{i}$ under different FUV fields (see values for the ISRF and the T Tauri and Herbig Ae/Be stellar radiation fields in Table 2). We note that the photodesorption yield of $\mathrm{CO}_{2}$, for example, is almost one order of magnitude higher under the ISRF than under a Herbig Ae/Be stellar field.

- Cosmic-ray induced desorption. This mechanism is driven by the impact of cosmic rays on dust grains. The energy deposited on dust grains upon impact of relativistic heavy nuclei of iron results in a local heating that induces the thermal desorption of the ice molecules present in the heated region. According to Hasegawa \& Herbst (1993), the desorption rate induced by cosmic rays, in units of $\mathrm{s}^{-1}$, of a species $i$ is given by

$R_{i}^{\mathrm{crd}}=3.16 \times 10^{-19} R_{i}^{\mathrm{thd}}(70 \mathrm{~K})$,

where the numerical factor stands for the fraction of the time spent by grains in the vicinity of a temperature of $70 \mathrm{~K}$, at which much of the desorption is assumed to occur in the formalism of Hasegawa \& Herbst (1993), and is derived adopting the Fe cosmic ray flux estimated by Léger et al. (1985) for the local interstellar medium and dust grains with a radius of $0.1 \mu \mathrm{m}$. The term $R_{i}^{\text {thd }}(70 \mathrm{~K})$ is the rate of thermal desorption of species $i$, given by Eq. (10), evaluated at $70 \mathrm{~K}$. The desorption rate $R_{i}^{\mathrm{crd}}$ scales with the cosmic-ray ionization rate.

\subsubsection{Formation of $\mathrm{H}_{2}$ on grain surfaces}

The kinetics of $\mathrm{H}_{2}$ formation on grain surfaces in interstellar space is usually described as

$\frac{d n\left(\mathrm{H}_{2}\right)}{d t}=R_{f} n_{\mathrm{H}} n(\mathrm{H})$

where $n_{\mathrm{H}}$ is the volume density of $\mathrm{H}$ nuclei, $n(\mathrm{H})$ and $n\left(\mathrm{H}_{2}\right)$ are the volume densities of neutral $\mathrm{H}$ atoms and $\mathrm{H}_{2}$ molecules, and $R_{f}$ is the formation rate parameter for which usually the canonical value of $3 \times 10^{-17} \mathrm{~cm}^{3} \mathrm{~s}^{-1}$ derived by Jura (1975) for diffuse interstellar clouds is adopted. Here, we evaluate $R_{f}$ as

$R_{f}=\frac{1}{2} S_{\mathrm{H}} \epsilon_{\mathrm{H}_{2}} v_{\mathrm{H}}\left\langle\sigma_{d} n_{d}\right\rangle \frac{1}{n_{\mathrm{H}}}$ 
where $v_{\mathrm{H}}$ is the thermal velocity of $\mathrm{H}$ atoms, evaluated as $\sqrt{3 k T_{k} / m_{\mathrm{H}}}$. The sticking coefficient of $\mathrm{H}$ atoms $S_{\mathrm{H}}$ depends on the gas kinetic temperature $T_{k}$ and is evaluated through the expression

$S_{\mathrm{H}}\left(T_{k}\right)=S_{0} \frac{\left(1+\beta T_{k} / T_{0}\right)}{\left(1+T_{k} / T_{0}\right)^{\beta}}$,

where we have adopted $S_{0}=1, T_{0}=25 \mathrm{~K}$, and $\beta=2.5$, based on the experimental study carried out by Chaabouni et al. (2012) for a silicate surface. The recombination efficiency $\epsilon_{\mathrm{H}_{2}}$ in Eq. (14) depends on the dust temperature $T_{d}$ according to the expression derived by Cazaux \& Tielens $(2002 a, b)$ and is evaluated with the parameters provided by Cazaux \& Tielens (2002a) in their Table 1, with an updated value of $12200 \mathrm{~K}$ for the desorption energy of chemisorbed $\mathrm{H}$, as calculated by Goumans et al. (2009) for an olivine surface. Currently, the kinetics of grain-surface $\mathrm{H}_{2}$ formation considered in the model accounts for the Langmuir-Hinshelwood mechanism. In the future it will be worth considering also the Eley-Rideal mechanism, which is expected to increase the $\mathrm{H}_{2}$ formation efficiency at high gas temperatures (e.g., Le Bourlot et al. 2012; Bron et al. 2014).

\section{Results}

In this section we present the calculated abundance distributions of various molecules in our fiducial T Tauri and Herbig Ae/Be disk models, and compare them with available constraints from observations, specifically highlighting the similarities and differences between both types of disks. We focus on molecules that have been observed in disks at IR or (sub-)millimeter wavelengths. Detected species in disks have been summarized by Dutrey et al. (2014). In Table 3 we provide an updated comprehensive summary of the molecules observed in T Tauri and Herbig Ae/Be disks. We first concentrate on molecules observed through IR observations, which are sensitive to the hot inner disk: $\mathrm{H}_{2} \mathrm{O}$ and $\mathrm{OH}$ (Sect. 3.1), and simple organics such as $\mathrm{C}_{2} \mathrm{H}_{2}$, $\mathrm{HCN}, \mathrm{CH}_{4}$, and $\mathrm{CO}_{2}$ (Sect. 3.2). We then focus on molecules observed at (sub-)millimeter wavelengths, which trace the outer disk: the radicals $\mathrm{C}_{2} \mathrm{H}$ and $\mathrm{CN}$ (Sect. 3.3) and other organic molecules with a certain complexity, such as $\mathrm{H}_{2} \mathrm{CO}$ (Sect. 3.4), the sulfur-bearing molecules CS and SO (Sect. 3.5), and molecular ions (Sect. 3.6). We finally discuss the abundance distributions of ices, for which most observational constraints consist of determining the location of the CO snow line (Sect. 3.7). Abundances are mostly expressed as column densities because this is the quantity provided by most observational studies. Nonetheless, sometimes we use the term fractional abundance, which hereafter refers to the abundance relative to the total number of $\mathrm{H}$ nuclei.

\subsection{Water and hydroxyl radical}

In recent years, near-IR to sub-millimeter observations have provided important constraints on the presence of water and its related radical $(\mathrm{OH})$ in protoplanetary disks. At near- and mid-IR wavelengths, the spectra of disks around T Tauri stars show emission of hot $\mathrm{H}_{2} \mathrm{O}$ and $\mathrm{OH}$ arising from the inner disk ( $<$ a few au) atmosphere (Carr et al. 2004; Carr \& Najita 2008, 2011, 2014; Salyk et al. 2008, 2011; Pontoppidan et al. 2010a,b; Mandell et al. 2012; Sargent et al. 2014; Banzatti et al. 2017), while in disks around Herbig Ae/Be stars, emission by $\mathrm{OH}$ is relatively common but there is a striking lack of $\mathrm{H}_{2} \mathrm{O}$ emission
(Mandell et al. 2008; Pontoppidan et al. 2010a; Fedele et al. 2011; Salyk et al. 2011; Banzatti et al. 2017). Far-IR observations with Herschel/PACS have essentially confirmed that the water detection rate is much higher in T Tauri disks than in disks around Herbig Ae/Be stars (Riviere-Marichalar et al. 2012; Meeus et al. 2012; Fedele et al. 2012, 2013). Therefore, IR observations suggest that water could be intrinsically less abundant in disks around Herbig Ae/Be stars than around T Tauri stars. Such a trend is however not corroborated by our models.

As can be seen in Fig. 4, in the T Tauri disk model, water is present with fractional abundances of $\sim 10^{-4}$ in a surface layer at $A_{V} \sim 1$ in the inner $\sim 10$ au from the star, while in the Herbig $\mathrm{Ae} / \mathrm{Be}$ disk, the warmer temperatures cause the region of high $\mathrm{H}_{2} \mathrm{O}$ abundance to extend radially beyond 10 au and vertically down to the midplane. In the inner disk, water becomes very abundant in regions warmer than $\sim 200 \mathrm{~K}$, where the reaction $\mathrm{OH}+\mathrm{H}_{2}$ is activated, and sufficiently shielded from FUV photons, while $\mathrm{OH}$ is present in a thin layer on top of $\mathrm{H}_{2} \mathrm{O}$ resulting from its photodissociation. In our generic T Tauri disk model, the inner midplane regions are not warm enough to sustain a highwater abundance, and thus most $\mathrm{H}_{2} \mathrm{O}$ (and all $\mathrm{OH}$ ) are present in upper layers. We, however, note that the presence of water vapor in the inner midplane is very sensitive to the temperature and that other T Tauri disk models (e.g., Walsh et al. 2015) find high $\mathrm{H}_{2} \mathrm{O}$ abundances in these regions. Therefore, the model predicts that as the stellar luminosity increases and the disk becomes warmer, water vapor becomes more abundant.

The vertical column densities calculated for $\mathrm{H}_{2} \mathrm{O}$ and $\mathrm{OH}$ in the IR-observable atmosphere ${ }^{12}$ of the inner T Tauri disk are $10^{17}-10^{18} \mathrm{~cm}^{-2}$ and $\sim 10^{15} \mathrm{~cm}^{-2}$, respectively, which are in the low range of observed values (see left panel in Fig. 5). The calculated $\mathrm{OH} / \mathrm{H}_{2} \mathrm{O}$ column density ratio in the inner disk, $10^{-2}-10^{-3}$, is between the values derived in AA Tau, DR Tau, and AS 205A (0.1-0.3; Carr \& Najita 2008; Salyk et al. 2008) and that found from a study of a much larger sample of T Tauri disks $\left(\sim 10^{-3}\right.$; Salyk et al. 2011). Previous chemical models of inner T Tauri disks (Agúndez et al. 2008; Walsh et al. 2015) find $\mathrm{H}_{2} \mathrm{O}$ and $\mathrm{OH}$ column densities and ratios of the same order of magnitude as the ones calculated by us. In summary, chemical models of T Tauri disks predict the existence of a significant reservoir of hot water in the inner regions (formed by warm gas-phase chemistry) and smaller amounts of $\mathrm{OH}$ (formed by FUV photodissociation of water), with numbers that are roughly in agreement with those derived from IR observations.

In disks around Herbig Ae/Be stars, $\mathrm{OH}$ has been detected in about a dozen objects with a broad range of column densities (see Table 3). In our Herbig Ae/Be disk model, the calculated vertical column density of $\mathrm{OH}$ is $\sim 10^{15} \mathrm{~cm}^{-2}$ across the first $10 \mathrm{au}$, decreasing down to $\sim 10^{13} \mathrm{~cm}^{-2}$ in the outer disk, in the low range of values derived from observations (see right panel in Fig. 5). Walsh et al. (2015) calculate somewhat higher values for the inner $10 \mathrm{au}, 10^{16}-10^{17} \mathrm{~cm}^{-2}$, more in line with the high range of observed values. Water has been convincingly detected at IR wavelengths only around one Herbig star, HD 163296 (Meeus et al. 2012; Fedele et al. 2012). The $\mathrm{H}_{2} \mathrm{O}$ and $\mathrm{OH}$ column densities derived in this disk are similar, in the range $10^{14}-10^{15} \mathrm{~cm}^{-2}$, and the emitting region for both species is constrained to be 1520 au from the star. In our Herbig Ae/Be disk model, the column density of water is very large in the inner disk (out to $\sim 4 \mathrm{au}$ ), where it is very abundant in the midplane (see right panels in Figs. 4 and 5), and experiences a sharp abundance decline with

12 We consider the IR-observable atmosphere to extend down to the $A_{V}=10$ layer, where the optical depth at $10 \mu \mathrm{m}$ is of the order of unity. 
Table 3. Summary of molecules (other than $\mathrm{H}_{2}$ and $\mathrm{CO}$ ) observed in disks and abundances derived.

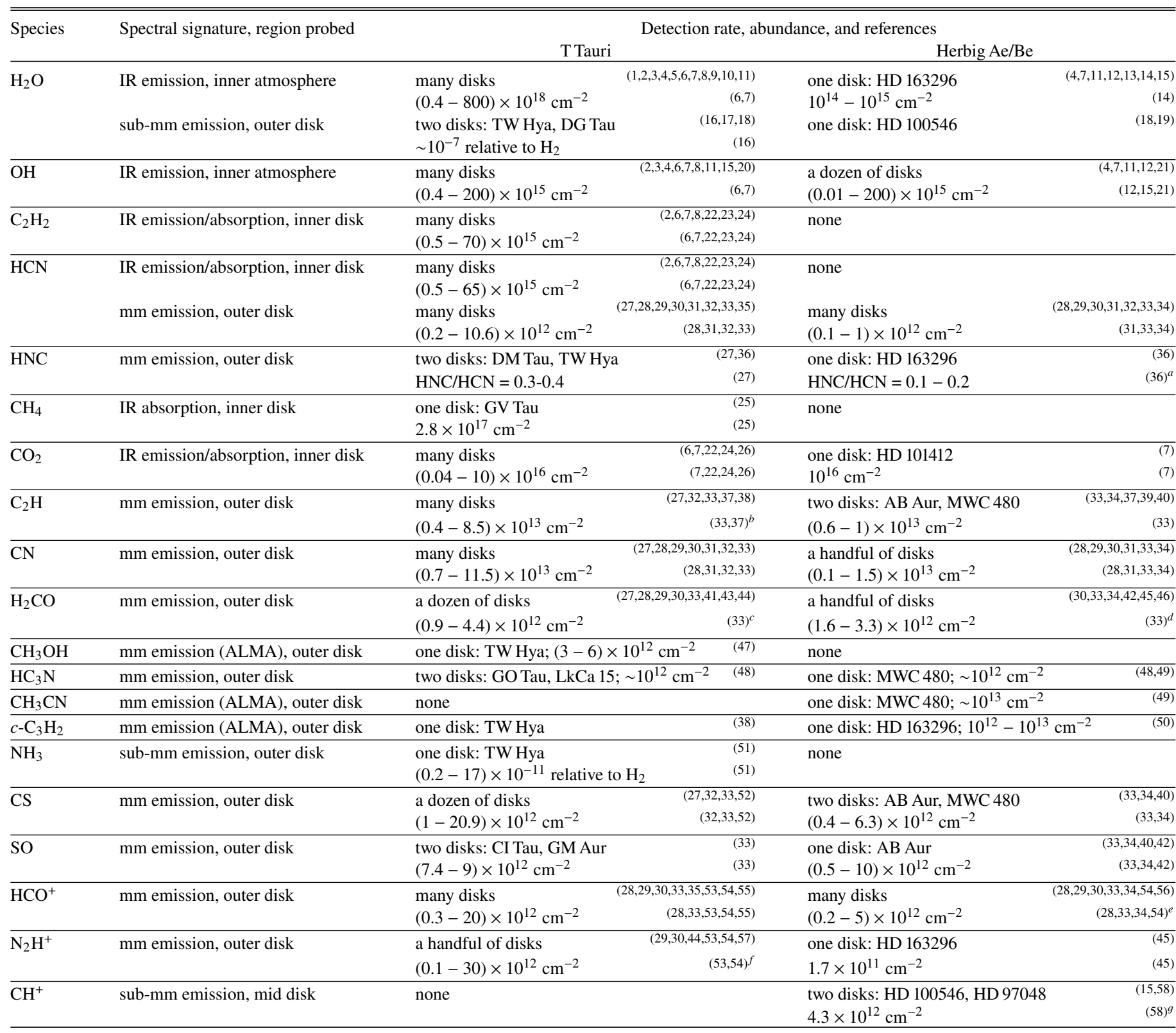

Notes. ${ }^{(a)}$ Values are line intensity ratios rather than abundance ratios. ${ }^{(b)}$ Out of this range, Kastner et al. $(2014)$ derive $N\left(\mathrm{C}_{2} \mathrm{H}\right)=$ $5.1 \times 10^{15} \mathrm{~cm}^{-2}$ in TW Hya. ${ }^{(c)}$ Out of this range, Aikawa et al. (2003) derive $N\left(\mathrm{H}_{2} \mathrm{CO}\right)=(7.2-19) \times 10^{12} \mathrm{~cm}^{-2}$ in $\mathrm{LkCa} 15$. ${ }^{(d)}$ Carney et al. (2017) derive a $\mathrm{H}_{2} \mathrm{CO}$ abundance of $(2-5) \times 10^{-12}$ relative to $\mathrm{H}_{2}$ in $\mathrm{HD} 163296$. ${ }^{(e)}$ Out of this range, Mathews et al. (2013) derive $N\left(\mathrm{HCO}^{+}\right)=1.5 \times 10^{14} \mathrm{~cm}^{-2}$ in $\mathrm{HD} 163296 .{ }^{(f)}$ Out of this range, Qi et al. $(2013 \mathrm{c})$ derive $N\left(\mathrm{~N}_{2} \mathrm{H}^{+}\right)=10^{14}-$ $10^{15} \mathrm{~cm}^{-2}$ in TW Hya. ${ }^{(g)}$ In the same object, HD 100546, Fedele et al. (2013) derive $N\left(\mathrm{CH}^{+}\right)=10^{16}-10^{17} \mathrm{~cm}^{-2}$ for an emitting area inner to $50-70$ au. References. (1) Carr et al. (2004); (2) Carr \& Najita (2008); (3) Salyk et al. (2008); (4) Pontoppidan et al. (2010a); (5) Pontoppidan et al. (2010b); (6) Carr \& Najita (2011); (7) Salyk et al. (2011); (8) Mandell et al. (2012); (9) Riviere-Marichalar et al. (2012); (10) Sargent et al. (2014); (11) Banzatti et al. (2017); (12) Fedele et al. (2011); (13) Meeus et al. (2012); (14) Fedele et al. (2012); (15) Fedele et al. (2013); (16) Hogerheijde et al. (2011); (17) Podio et al. (2013); (18) Du et al. (2017) ; (19) van Dishoeck et al. (2014); (20) Carr \& Najita (2014); (21) Mandell et al. (2008); (22) Lahuis et al. (2006); (23) Gibb et al. (2007); (24) Bast et al. (2013); (25) Gibb \& Horne (2013); (26) Kruger et al. (2011); (27) Dutrey et al. (1997); (28) Thi et al. (2004); (29) Öberg et al. (2010); (30) Öberg et al. (2011); (31) Chapillon et al. (2012a); (32) Kastner et al. (2014); (33) Guilloteau et al. (2016); (34) Fuente et al. (2010); (35) Fuente et al. (2012); (36) Graninger et al. (2015); (37) Henning et al. (2010); (38) Bergin et al. (2016); (39) Schreyer et al. (2008); (40) Pacheco-Vázquez et al. (2015); (41) Aikawa et al. (2003); (42) Pacheco-Vázquez et al. (2016); (43) Loomis et al. (2015); (44) Öberg et al. (2017); (45) Qi et al. (2013a); (46) Carney et al. (2017); (47) Walsh et al. (2016); (48) Chapillon et al. (2012b); (49) Öberg et al. (2015); (50) Qi et al. (2013b); (51) Salinas et al. (2016); (52) Dutrey et al. (2011); (53) Qi et al. (2003); (54) Dutrey et al. (2007); (55) Teague et al. (2015); (56) Mathews et al. (2013); (57) Qi et al. (2013c); (58) Thi et al. (2011).

increasing radius. At $15-20$ au from the star, where water is no longer present in the midplane but in upper disk layers, the model yields $N\left(\mathrm{H}_{2} \mathrm{O}\right)=10^{15}-10^{16} \mathrm{~cm}^{-2}$ and $N(\mathrm{OH})=10^{14}-10^{15} \mathrm{~cm}^{-2}$, with a $\mathrm{OH} / \mathrm{H}_{2} \mathrm{O}$ ratio of $\sim 0.1$, values which are not far from those derived in the HD 163296 disk. In the Herbig Ae disk model of Walsh et al. (2015), at 10 au (the farthest radius studied by these authors) the column densities of $\mathrm{H}_{2} \mathrm{O}$ and $\mathrm{OH}$ are in the range $10^{16}-10^{17}$, with the $\mathrm{OH} / \mathrm{H}_{2} \mathrm{O}$ ratio approaching unity. The 

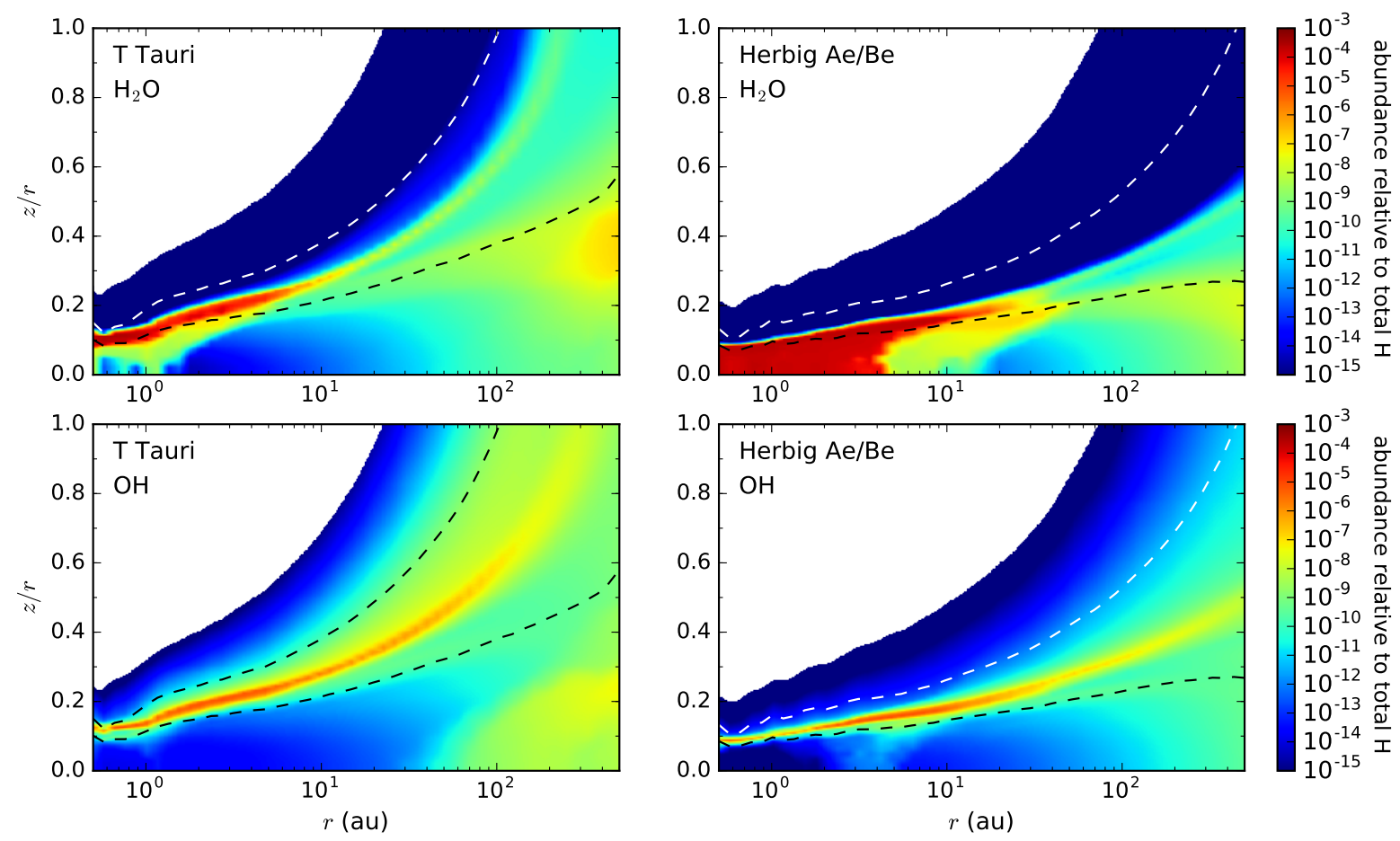

Fig. 4. Calculated distributions of $\mathrm{H}_{2} \mathrm{O}$ and $\mathrm{OH}$ as a function of radius $r$ and height over radius $z / r$ for the T Tauri (left panel) and Herbig Ae/Be (right panel) disks. The dashed lines indicate the location where $A_{V}$ in the outward vertical direction takes values of 0.01 and 1 .
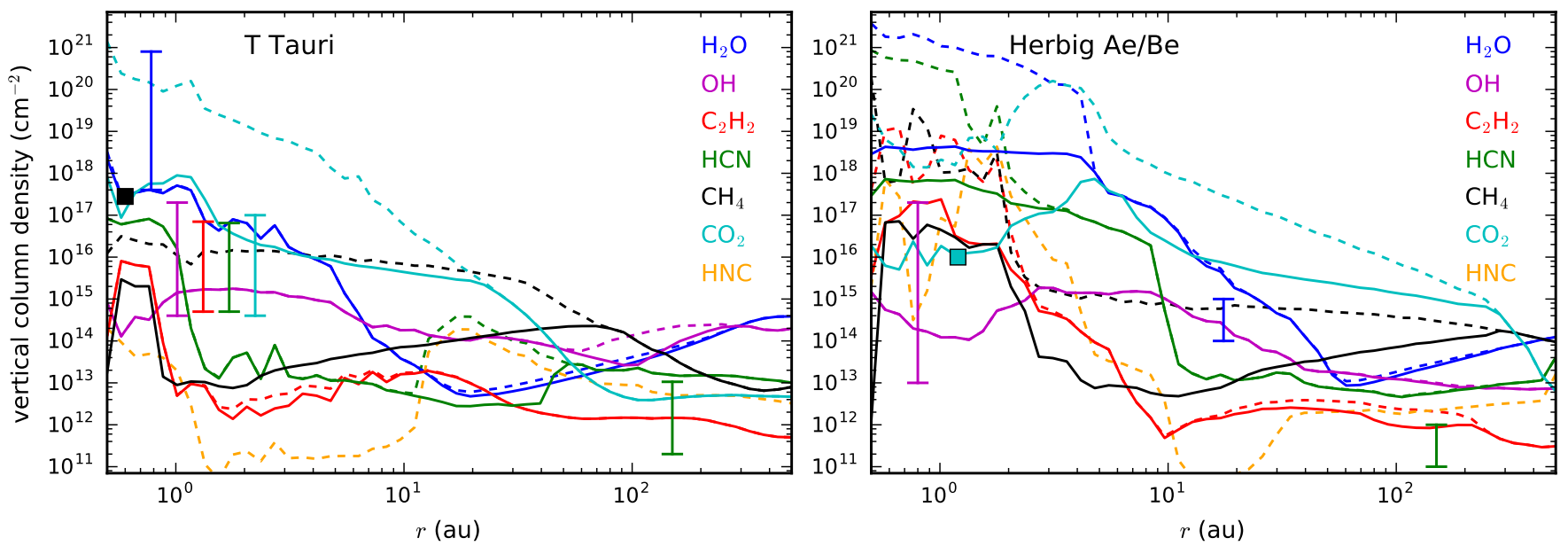

Fig. 5. Calculated vertical column densities down to the $A_{V}=10$ surface (solid lines) and down to the midplane (dashed lines) of $\mathrm{H}_{2} \mathrm{O}, \mathrm{OH}$, and simple organics as a function of radius $r$ for the T Tauri (left panel) and Herbig Ae/Be (right panel) disks. For HNC, only the total column density down to the midplane is shown. Column densities derived from observations are indicated by vertical lines (when there are ranges of values) or by squares (when only one value is available), with their radial locations corresponding to the approximate region probed by observations (see Table 3).

model of Walsh et al. (2015) and ours do a reasonable job at explaining the order of magnitude of the water and $\mathrm{OH}$ observations of HD 163296. It however remains puzzling to explain the extremely low detection rate of water in Herbig Ae/Be disks, as compared with T Tauri disks, taking into account the fact that chemical models (Walsh et al. 2015 and this work) predict that in disks around Herbig stars, water should be even more abundant than in T Tauri disks. Several explanations have been proposed (see Antonellini et al. 2016 and references therein), most of which are related to observational aspects (e.g., the higher level of IR continuum in Herbig Ae/Be disks and the lower sensitivity reached for detection of emission lines above the continuum) than to substantive differences in the chemistry between disks around low- and intermediate-mass pre-main sequence stars.

Our model predicts that the reservoir of hot water present in the inner regions of disks around T Tauri and Herbig Ae/Be stars vanishes typically beyond 10 au from the star, owing to thermal deactivation of the water-forming reaction $\mathrm{OH}+\mathrm{H}_{2}$ and to freeze-out onto dust grains. This drastic decline of various orders of magnitude in the abundance and column density of water (see Figs. 4 and 5) has been observationally probed by mid- to far-IR observations in a few protoplanetary disks (Blevins et al. 2016). The model, however, predicts that there exists an additional reservoir of cold water in the outer parts 
of T Tauri and Herbig Ae/Be disks, typically beyond 100 au and at intermediate heights (see Fig. 4). Water in these regions arises from the FUV photodesorption of water ice and reaches peak fractional abundances of $\sim 10^{-7}$, with typical vertical column densities of the order of $10^{13}-10^{14} \mathrm{~cm}^{-2}$ (see Fig. 5). This outer reservoir of water is also predicted by previous chemical models of T Tauri disks that include photodesorption (e.g., Willacy \& Langer 2000; Woitke et al. 2009; Semenov et al. 2011; Walsh et al. 2012), and has been detected with Herschel/HIFI in the T Tauri disks TW Hya and DG Tau (Hogerheijde et al. 2011; Podio et al. 2013) and in the Herbig Be disk HD 100546 (van Dishoeck et al. 2014; Du et al. 2017), although it remains elusive in many other protoplanetary disks (Bergin et al. 2010; Du et al. 2017). It is remarkable that the detection rate of water at sub-millimeter wavelengths is not so different between T Tauri and Herbig Ae/Be disks as it is at IR wavelengths (see Table 3), which suggests that the low detection rate of $\mathrm{H}_{2} \mathrm{O}$ IR emission in Herbig objects may not be due to an intrinsic deficit of water with respect to T Tauri systems.

\subsection{Simple organics: $\mathrm{C}_{2} \mathrm{H}_{2}, \mathrm{HCN}, \mathrm{CH}_{4}$, and $\mathrm{CO}_{2}$}

It is known that there exists an important reservoir of simple organic molecules in protoplanetary disks. Thanks to observations at near- and mid-IR wavelengths, lines of molecules such as acetylene, hydrogen cyanide, methane, and carbon dioxide have been detected in absorption (Lahuis et al. 2006; Gibb et al. 2007; Gibb \& Horne 2013; Bast et al. 2013) and in emission (Carr \& Najita 2008, 2011; Salyk et al. 2011; Kruger et al. 2011; Mandell et al. 2012; Najita et al. 2013; Pascucci et al. 2013). These observations probe hot gas located in the inner (a few au) disk, where these molecules are found in large abundances. It is noteworthy that the vast majority of IR detections of simple organics correspond to disks around T Tauri stars rather than to disks around Herbig $\mathrm{Ae} / \mathrm{Be}$ stars, where neither $\mathrm{C}_{2} \mathrm{H}_{2}, \mathrm{HCN}$, nor $\mathrm{CH}_{4}$ are detected, and only $\mathrm{CO}_{2}$ has been detected in one disk, HD 101412 (Salyk et al. 2011). It is therefore tempting to speculate that Herbig Ae/Be disks are less rich in simple organics than disks around T Tauri stars. However, similarly to the case of $\mathrm{H}_{2} \mathrm{O}$, we do not find such a trend in our models.

In our T Tauri disk model, $\mathrm{C}_{2} \mathrm{H}_{2}, \mathrm{HCN}$, and $\mathrm{CH}_{4}$ are formed with high fractional abundances $\left(\mathrm{a}\right.$ few $\left.\times 10^{-5}\right)$ in the atmosphere $\left(A_{V} \sim 1\right)$ of the inner (within a few au) regions of the disk (see Fig. 6). Their synthesis is driven by FUV photochemistry in a warm gas (see Agúndez et al. 2008; Bast et al. 2013; Walsh et al. 2015). In the Herbig Ae/Be disk model, the region over which these molecules have large fractional abundances extends to larger radii compared to the $\mathrm{T}$ Tauri disk, as a consequence of the higher temperatures. Moreover, in the Herbig Ae/Be disk, $\mathrm{C}_{2} \mathrm{H}_{2}, \mathrm{HCN}$, and $\mathrm{CH}_{4}$ are formed abundantly in the inner midplane, where the synthesis is not related to photochemistry but to the fact that the chemical composition tends toward thermochemical equilibrium in these hot, dense, and FUV-shielded regions. Both the T Tauri and Herbig Ae/Be disk models show a progressive disappearance of simple organics as one moves radially from the star, in this order: $\mathrm{CH}_{4}, \mathrm{C}_{2} \mathrm{H}_{2}$, and $\mathrm{HCN}$. This behavior, already predicted by Agúndez et al. (2008), is a consequence of the requirements of temperature that each molecule has to activate its corresponding formation routes, with $\mathrm{CH}_{4}$ being the most demanding. Such a trend is also found in the disk models of Walsh et al. (2015) for $\mathrm{C}_{2} \mathrm{H}_{2}$ and $\mathrm{HCN}$. We note that $\mathrm{CH}_{4}$, unlike $\mathrm{C}_{2} \mathrm{H}_{2}$ and $\mathrm{HCN}$, is also predicted to be moderately abundant in cool midplane regions, where the synthesis is driven by ion-molecule routes. In these regions however the calculated abundance of $\mathrm{CH}_{4}$ could be especially uncertain if grain-surface chemistry (not included in our models) plays an important role.

The calculated vertical column densities of $\mathrm{C}_{2} \mathrm{H}_{2}$ and $\mathrm{HCN}$ in the IR-observable atmosphere of the inner $\mathrm{T}$ Tauri disk (within $1 \mathrm{au}$ from the star) are large, with maxima in the range $10^{15}-10^{17} \mathrm{~cm}^{-2}$, in good agreement with observed values (see left panel in Fig. 5). Observations of T Tauri disks indicate that $\mathrm{C}_{2} \mathrm{H}_{2}$ and $\mathrm{HCN}$ have similar abundances, although there is a significant dispersion, and that they are somewhat less abundant than water vapor $\left(\mathrm{C}_{2} \mathrm{H}_{2} / \mathrm{HCN}=0.04-20 ; \mathrm{HCN} / \mathrm{H}_{2} \mathrm{O}=10^{-3}\right.$ 10 ${ }^{-1}$; Lahuis et al. 2006; Gibb et al. 2007; Carr \& Najita 2011; Salyk et al. 2011; Mandell et al. 2012; Bast et al. 2013). These observed ratios are in line with the values found in the T Tauri disk model. Methane has only been detected in one T Tauri disk, GV Tau, in absorption (Gibb \& Horne 2013). These authors derive a column density of $2.8 \times 10^{17} \mathrm{~cm}^{-2}$ and a rotational temperature of $750 \mathrm{~K}$, which implies that the detected $\mathrm{CH}_{4}$ is distributed in the inner disk. In $\mathrm{GV} \mathrm{Tau}, \mathrm{CH}_{4}$ is somewhat more abundant than $\mathrm{C}_{2} \mathrm{H}_{2}$ and $\mathrm{HCN}$. We note however that in DR Tau, the non detection of $\mathrm{CH}_{4}$ in emission implies that it has an abundance similar to or smaller than $\mathrm{C}_{2} \mathrm{H}_{2}$ and $\mathrm{HCN}$ (Mandell et al. 2012). In our T Tauri disk model, $\mathrm{CH}_{4}$ reaches a column density of the order of those of $\mathrm{C}_{2} \mathrm{H}_{2}$ and $\mathrm{HCN}$ in the IR-observable atmosphere of the inner disk (see left panel in Fig. 5). Neither $\mathrm{C}_{2} \mathrm{H}_{2}, \mathrm{HCN}$, or $\mathrm{CH}_{4}$ have been observed in Herbig Ae/Be disks, although our model predicts that they should be even more abundant than in T Tauri disks (see Figs. 5 and 6). Similar conclusions are found in the models by Walsh et al. (2015). The lack of simple organics in the spectra of Herbig Ae/Be disks has not been investigated to the extent of the lack of water, but it is likely that observational rather than chemical effects are at the origin of it.

Carbon dioxide has been extensively observed in T Tauri disks but only in one Herbig Ae/Be disk, where the derived column density is within the range of values found in T Tauri disks. In our models, $\mathrm{CO}_{2}$ is formed abundantly, mostly in the inner disk ( $<10$ au in the T Tauri disk and $<100$ au in the Herbig disk) and over most of the vertical structure (see Fig. 6). The formation of $\mathrm{CO}_{2}$ occurs in the gas-phase, mainly through the reaction $\mathrm{OH}+\mathrm{CO}$, and is less demanding in terms of temperature than the formation of $\mathrm{C}_{2} \mathrm{H}_{2}, \mathrm{HCN}$, and $\mathrm{CH}_{4}$. Therefore, $\mathrm{CO}_{2}$ extends over larger radii than the other simple organics. The calculated column density of $\mathrm{CO}_{2}$ in the IR-observable atmosphere of the inner $(<10 \mathrm{au}) \mathrm{T}$ Tauri disk is in the range $10^{16}-10^{18} \mathrm{~cm}^{-2}$ (see left panel in Fig. 5), in the high range of observed values. As with the other simple organics, according to the model there is no apparent chemical reason for a lower amount of $\mathrm{CO}_{2}$ in disks around Herbig Ae/Be stars than in T Tauri disks.

The simple organic molecules discussed here experience a drastic decline in their column densities with increasing radius, especially for $\mathrm{C}_{2} \mathrm{H}_{2}$ and $\mathrm{HCN}$ (see Fig. 5). This extended and cooler reservoir of simple organics can be probed at millimeter wavelengths in the case of polar molecules like HCN. In fact, $\mathrm{HCN}$ has been extensively characterized this way in protoplanetary disks (Dutrey et al. 1997; Thi et al. 2004; Fuente et al. 2010, 2012; Öberg et al. 2010, 2011; Chapillon et al. 2012a; Kastner et al. 2014; Guilloteau et al. 2016). Although the statistics of Herbig Ae disks is low, these studies suggest that T Tauri disks can retain somewhat larger $\mathrm{HCN}$ abundances in their outer parts than Herbig Ae disks (see Table 3). The column densities calculated for HCN beyond 100 au are $\sim 10^{13} \mathrm{~cm}^{-2}$ in both the T Tauri and the Herbig Ae/Be disks, in the high range of observed values 

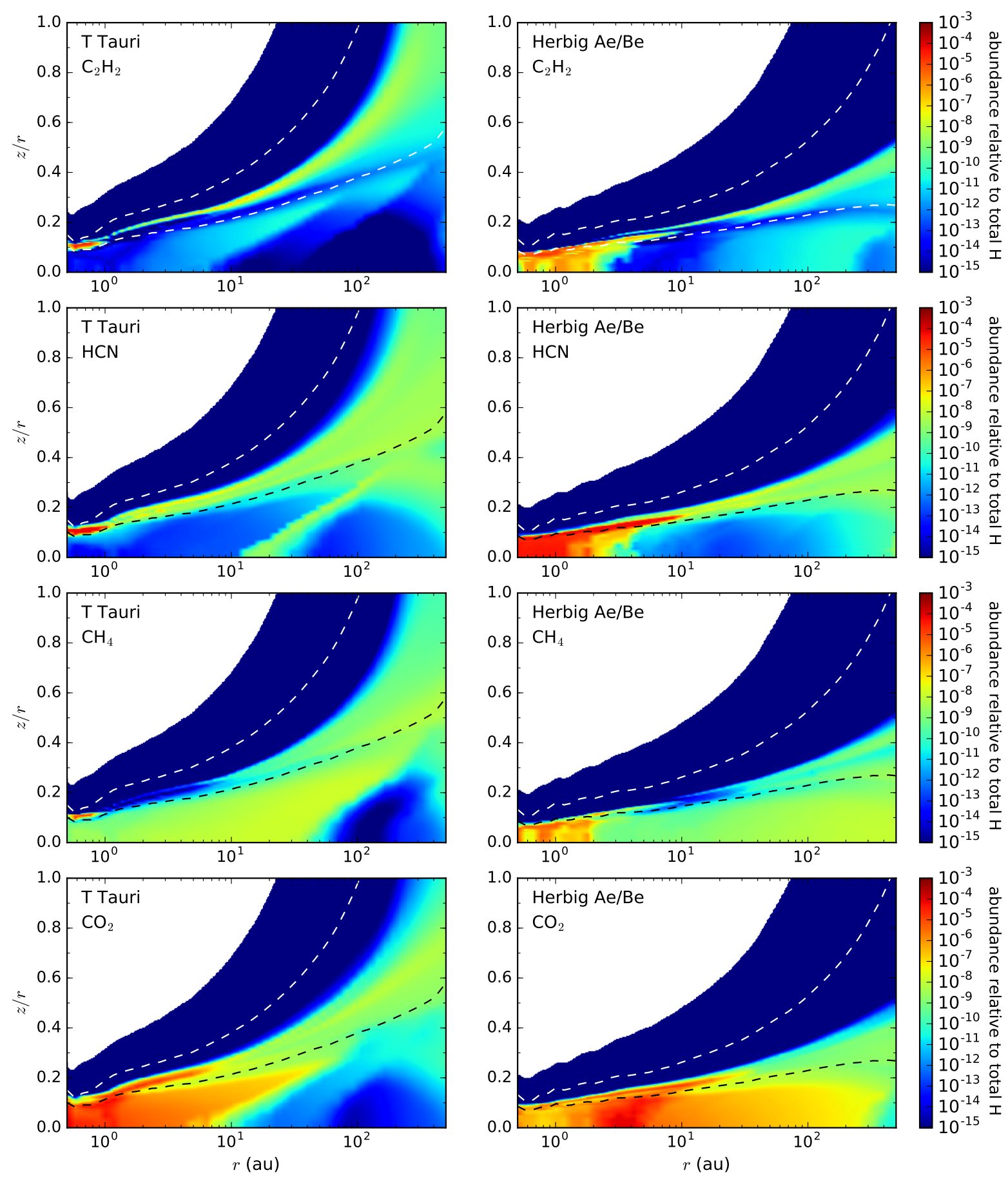

Fig. 6. As in Fig. 4 but for $\mathrm{C}_{2} \mathrm{H}_{2}, \mathrm{HCN}, \mathrm{CH}_{4}$, and $\mathrm{CO}_{2}$.

(see Fig. 5). The model predicts a slightly higher amount of HCN in the outer regions of the T Tauri disk compared to the Herbig disk. It is interesting to note that the contrary is found in the inner regions. The reason is that the chemical synthesis of $\mathrm{HCN}$ is different in nature in the hot inner disk than in the cool outer regions. In the outer disk ( $>100 \mathrm{au}), \mathrm{HCN}$ is mainly present at intermediate heights with fractional abundances of $\sim 10^{-8}$ relative to $\mathrm{H}_{2}$ (see Fig. 6). In these regions, $\mathrm{HCN}$ is formed by the same gas-phase chemical routes that operate in cold interstellar clouds, i.e., through ion-molecule reactions that lead to the precursor ion $\mathrm{HCNH}^{+}$, which by dissociative recombination yields $\mathrm{HCN}$ as well as its isomer HNC. Thus, both observations and our model suggest that $\mathrm{HCN}$ is somewhat more abundant in the outer regions of T Tauri disks compared to Herbig Ae/Be disks. We, however, caution that from an observational perspective, the statistics of Herbig Ae disks is low, and from a theoretical one, the difference is small and could result from the particular set of parameters adopted in the models.

Hydrogen isocyanide, closely related to $\mathrm{HCN}$ from a chemical point of view, has only been detected around the T Tauri stars DM Tau and TW Hya and the Herbig Ae star HD 163296 (Dutrey et al. 1997; Graninger et al. 2015). The lower detection rate of $\mathrm{HNC}$ in disks compared to its most stable isomer HCN suggests that $\mathrm{HNC}$ is less abundant than $\mathrm{HCN}$, although there is also a 

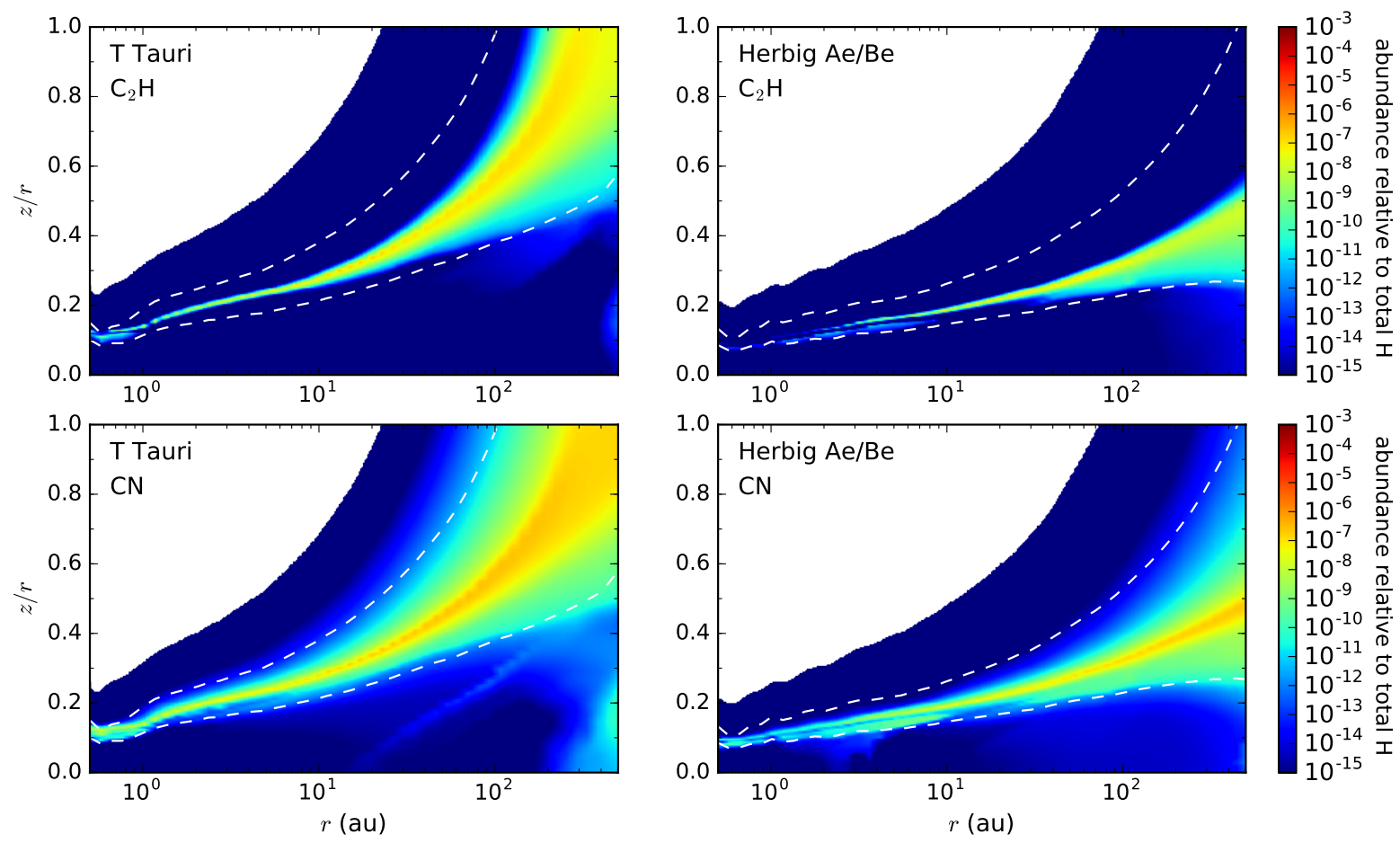

Fig. 7. As in Fig. 4 but for $\mathrm{C}_{2} \mathrm{H}$ and $\mathrm{CN}$.

selection effect as lines of HNC have not been targeted as often as those of HCN (e.g., Öberg et al. 2011; Guilloteau et al. 2016). Observations indicate that HNC is indeed somewhat less abundant than $\mathrm{HCN}$, with $\mathrm{HNC} / \mathrm{HCN}$ ratios in the range $0.1-0.4$ (see Table 3). These values may need to be revised down due to differences in the collisional excitation rate coefficients of $\mathrm{HCN}$ and HNC (Sarrasin et al. 2010). The calculated distribution of HNC approximately follows that of $\mathrm{HCN}$, but at a lower level of abundance (see Fig. 5). The model shows that there is a gap in the column density of HNC which occurs at 1-10 au in the T Tauri disk (shifted to larger radii in the Herbig Ae/Be disk). There is observational evidence of such a gap in the distribution of HNC from SMA interferometric observations of TW Hya (Graninger et al. 2015). While the presence of HNC in the hot inner disk is related to the existence of large amounts of $\mathrm{HCN}$, a fraction of which isomerizes to $\mathrm{HNC}$ in the hot gas phase, in the cool outer disk, HNC has an abundance of the order of that of HCN because the two isomers share the same chemical formation routes, with the molecular ion $\mathrm{HCNH}^{+}$as main precursor. The calculated $\mathrm{HNC} / \mathrm{HCN}$ ratio in the outer disk ( $>100 \mathrm{au}$ ) is $0.3-0.4$ in both the T Tauri and the Herbig Ae/Be disks, in line with the values derived from observations. Interferometric observations indicate that HNC does not extend out to radii as large as HCN in TW Hya (Graninger et al. 2015), a feature that is not predicted by our T Tauri disk model. A possible explanation could be related to an enhanced photodissociation cross section of $\mathrm{HNC}$ compared to that of $\mathrm{HCN}$, something that is suggested by theoretical calculations (Chenel et al. 2016; Aguado et al. 2017).

\subsection{Radicals $\mathrm{C}_{2} \mathrm{H}$ and $\mathrm{CN}$}

The radicals $\mathrm{C}_{2} \mathrm{H}$ and $\mathrm{CN}$, usually considered as good tracers of regions affected by FUV radiation such as PDRs, are among the most conspicuous molecules detected in protoplanetary disks at millimeter wavelengths. These observations probe the emission from the outer disk, out to some hundreds of astronomical units from the star. Constraints on the abundances of $\mathrm{C}_{2} \mathrm{H}$ and $\mathrm{CN}$ mainly come from observations of a few protoplanetary disks, the widely studied T Tauri disks DM Tau, LkCa 15, and TW Hya, and the Herbig Ae disks HD 163296, MWC 480, and AB Aur (Thi et al. 2004; Schreyer et al. 2008; Henning et al. 2010; Fuente et al. 2010; Chapillon et al. 2012a; Kastner et al. 2014) and from the more recent study of Guilloteau et al. (2016), in which a larger sample of disks was observed. These studies suggest that $\mathrm{C}_{2} \mathrm{H}$ and $\mathrm{CN}$ can reach higher abundances in T Tauri disks than in Herbig Ae disks (see Table 3). This would be in line with the general observational finding of a higher detection rate of molecules in T Tauri disks compared to Herbig Ae disks (e.g., Öberg et al. 2011; Guilloteau et al. 2016). However, the confirmation of such hypothesis is still hampered by the low statistics of Herbig Ae disks observed to date. Observations indicate that $\mathrm{C}_{2} \mathrm{H}$ is present with an abundance similar to that of $\mathrm{CN}$ in most disks, with $\mathrm{C}_{2} \mathrm{H} / \mathrm{CN}$ ratios in the range 0.2-3.5 (Guilloteau et al. 2016), and no significant difference between T Tauri and Herbig Ae disks. It is also found that the $\mathrm{CN}$ radical is significantly more abundant than $\mathrm{HCN}$ in all observed disks, with $\mathrm{CN} / \mathrm{HCN}$ abundance ratios in the range 4-30 (Guilloteau et al. 2016), and again no substantive difference between T Tauri and Herbig Ae disks. In summary, observations at millimeter wavelengths tell us that T Tauri disks can retain larger abundances of $\mathrm{C}_{2} \mathrm{H}$ and $\mathrm{CN}$ in their outer regions than Herbig Ae disks, and that in round numbers the $\mathrm{C}_{2} \mathrm{H} / \mathrm{CN}$ ratio is $\sim 1$ and the $\mathrm{CN} / \mathrm{HCN}$ ratio is $\sim 10$ in both types of disk. It is also interesting to note that recent ALMA observations have found that $\mathrm{C}_{2} \mathrm{H}$ emission shows a ring-like distribution in the TW Hya and DM Tau disks (Bergin et al. 2016).

In the T Tauri and Herbig Ae/Be disk models, $\mathrm{C}_{2} \mathrm{H}$ and $\mathrm{CN}$ are essentially located in a relatively thin layer in the disk surface, between the $A_{V}=1$ and $A_{V}=0.01$ layers (see Fig. 7), with vertical column densities of the order of $10^{13} \mathrm{~cm}^{-2}$ in the outer disk (see Fig. 8). Protoplanetary disks can be seen as a $\mathrm{PDR}$ with the typical layered structure $\mathrm{CO} / \mathrm{C} / \mathrm{C}^{+}$along the vertical direction, and $\mathrm{C}_{2} \mathrm{H}$ and $\mathrm{CN}$ become abundant in the layer where neutral atomic carbon reaches its maximum abundance. 
The formation of $\mathrm{C}_{2} \mathrm{H}$ and $\mathrm{CN}$ is therefore associated to the disk PDR and to the availability of atomic $\mathrm{C}$ in a FUV illuminated gas. Our results regarding the T Tauri disk are similar to those of previous models of disks around low-mass young stars (e.g., Aikawa \& Herbst 1999b; Aikawa et al. 2002; Willacy \& Langer 2000; van Zadelhoff et al. 2003; Willacy et al. 2006; Walsh et al. 2010; Semenov et al. 2011). There are some differences between the T Tauri and the Herbig Ae/Be disks. The higher gravity of the Herbig Ae/Be star causes the layer containing $\mathrm{C}_{2} \mathrm{H}$ and $\mathrm{CN}$ to be more compressed toward lower heights (see Fig. 7), although the most significant difference is that the calculated column densities of $\mathrm{C}_{2} \mathrm{H}$ and $\mathrm{CN}$ in the outer disk are somewhat lower in the Herbig Ae/Be disk (see Fig. 8). The reason is that the Herbig disk is illuminated by a more intense FUV field from the star than the T Tauri disk, and this narrows the photochemically active layer and limits the ability of photochemistry to build molecules, resulting in smaller amounts of molecules specifically formed by the action of photochemistry. This is a general difference between disks around T Tauri and Herbig stars that is better appreciated in the cool outer disk (>100 au), where warm gas-phase chemistry is inhibited and thus cannot counterbalance the effect of FUV photons. For example, beyond $100 \mathrm{au}$, the radical $\mathrm{OH}$ (a species typically formed under the action of photochemistry) is significantly more abundant in the T Tauri disk than in the Herbig disk (see Fig. 5). In the case of $\mathrm{C}_{2} \mathrm{H}$ and $\mathrm{CN}$, the fact that the abundances are lower in the Herbig disk is linked to a slight defficiency in the abundance of neutral atomic carbon, which is a key starting point to form both $\mathrm{C}_{2} \mathrm{H}$ and $\mathrm{CN}$. The calculated column densities of $\mathrm{C}_{2} \mathrm{H}$ and $\mathrm{CN}$ are in line with the values derived from observations. Moreover, the slight overabundance (a factor of a few) of these radicals in the T Tauri disk compared to the Herbig Ae/Be disk is in line the observational suggestion that $\mathrm{T}$ Tauri disks can retain higher abundances of these two radicals. Another salient feature of the model is that the column densities of both $\mathrm{C}_{2} \mathrm{H}$ and $\mathrm{CN}$ increase with increasing radius in both the $\mathrm{T}$ Tauri and the Herbig Ae/Be disks. The predicted inner gap could be consistent with the ringlike distribution found for $\mathrm{C}_{2} \mathrm{H}$ in the TW Hya and DM Tau disks (Bergin et al. 2016). These authors however propose a scenario in which the ring morphology observed for $\mathrm{C}_{2} \mathrm{H}$, and also for cyclic $\mathrm{C}_{3} \mathrm{H}_{2}$, is related to the evolution of ice-coated dust due to the combined effect of coagulation, gravitational settling, and drift.

The model indicates that $\mathrm{HCN}$ is much more abundant than $\mathrm{CN}$ in the inner regions of both types of disk, although as one moves away from the star, the $\mathrm{CN} / \mathrm{HCN}$ ratio increases, reaching values above unity at radii $>50$ au (see Fig. 8). The overabundance of $\mathrm{CN}$ with respect to $\mathrm{HCN}$ in the outer disk is in agreement with observations. According to the model, in the outer disk, $\mathrm{C}_{2} \mathrm{H}$ and $\mathrm{CN}$ do not spatially coexist with $\mathrm{HCN}$, the radicals being exclusively present in a relatively thin layer at the disk surface while $\mathrm{HCN}$ is located at lower heights. There is some controversy regarding the region where $\mathrm{C}_{2} \mathrm{H}$ and $\mathrm{CN}$ are present in T Tauri disks because millimeter observations derive low excitation temperatures $(\lessgtr 10 \mathrm{~K})$ for these two radicals (Henning et al. 2010; Chapillon et al. 2012a; Hily-Blant et al. 2017), which suggest that the emission could arise from cold midplane regions rather than from the warm disk surface. If true, this would be in strong disagreement with the predictions of our model and previous chemical models of T Tauri disks (e.g., Aikawa \& Herbst 1999b; Aikawa et al. 2002; Willacy \& Langer 2000; van Zadelhoff et al. 2003; Willacy et al. 2006; Walsh et al. 2010; Semenov et al. 2011), which also locate the radicals $\mathrm{C}_{2} \mathrm{H}$ and $\mathrm{CN}$ well above the midplane. Observations and chemical models of T Tauri disks could still be reconciled if in the outer regions ( $>100 \mathrm{au}$ ) of the surface layer containing $\mathrm{C}_{2} \mathrm{H}$ and $\mathrm{CN}$ (where densities are in the range $10^{5}-10^{6} \mathrm{~cm}^{-3}$ and gas kinetic temperatures are between 30 and $100 \mathrm{~K}$ ) these two radicals are subthermally excited. We note however that this could have implications for the column densities derived from observations under the assumption of local thermal equilibrium (LTE). Dedicated non-LTE excitation and radiative transfer calculations (e.g., Aikawa et al. 2002) and comparison with millimeter observations are needed to shed light on this issue.

\subsection{Organic molecules of a certain complexity}

In this section we briefly comment on various organic molecules observed in disks that are more complex than those treated in Sect. 3.2. We refer to $\mathrm{H}_{2} \mathrm{CO}$ and $\mathrm{CH}_{3} \mathrm{OH}$, the hydrogenation descendants of carbon monoxide, the cyanides $\mathrm{HC}_{3} \mathrm{~N}$ and $\mathrm{CH}_{3} \mathrm{CN}$, and the cyclic isomer of the hydrocarbon $\mathrm{C}_{3} \mathrm{H}_{2}$.

Formaldehyde has been quite commonly observed in protoplanetary disks, with a higher detection rate in T Tauri disks than in Herbig Ae disks (Öberg et al. 2010, 2011; Guilloteau et al. 2016), but very similar column densities, that is, a few times $10^{12} \mathrm{~cm}^{-2}$, in both types of disk (see Table 3). Interferometric observations of the disks DM Tau, TW Hya, and HD 163296 have provided interesting constraints on the distribution and origin of $\mathrm{H}_{2} \mathrm{CO}$ (Qi et al. 2013a; Loomis et al. 2015; Öberg et al. 2017; Carney et al. 2017). These observations point to the presence of an inner component, consistent with gas-phase formation, and an abundance enhancement in the outer disk (beyond the $\mathrm{CO}$ snow line) resulting from formation on grain surfaces by hydrogenation of $\mathrm{CO}$ ice followed by desorption. The recent detection of methanol in the TW Hya disk (Walsh et al. 2016) also points to a similar outer disk origin driven by the hydrogenation of $\mathrm{CO}$ ice. In our model, which does not include grain-surface chemistry, calculated column densities in the outer regions of the T Tauri and Herbig Ae/Be disks are around $10^{12} \mathrm{~cm}^{-2}$ for $\mathrm{H}_{2} \mathrm{CO}$ (i.e., not far from observed values), but vanishingly small for $\mathrm{CH}_{3} \mathrm{OH}$. We note that if most of the $\mathrm{H}_{2} \mathrm{CO}$ and $\mathrm{CH}_{3} \mathrm{OH}$ in the outer disk indeed comes from $\mathrm{CO}$ ice through grain-surface chemistry, one should expect a significant differentiation between T Tauri and Herbig disks because the latter are warmer and should have a lower reservoir of the precursor $\mathrm{CO}$ ice.

Other relatively large organic molecules observed in disks are $\mathrm{HC}_{3} \mathrm{~N}, \mathrm{CH}_{3} \mathrm{CN}$, and cyclic $\mathrm{C}_{3} \mathrm{H}_{2}$ (see Table 3). Observations indicate that these molecules are present in the outer disk, out to a few hundred astronomical units. In these regions, our model predicts column densities below the observed values by one to two orders of magnitude. Calculated column densities for these molecules are quite different among chemical models in the literature. For example, for $\mathrm{HC}_{3} \mathrm{~N}$, Chapillon et al. (2012b) calculate column densities one to two orders of magnitude above the observed ones, while Walsh et al. (2014) find values ten times lower than observed. The dispersion of calculated column densities between different chemical models and the poor agreement with observations suggests that the chemistry of these moderately complex molecules is not yet as robust as for smaller species.The probable role of grain-surface reactions in regulating their abundances makes it worth revisiting their chemistry in T Tauri and Herbig Ae disks with an expanded chemical network including grain-surface chemistry.

We note that our model predicts that organic molecules like $\mathrm{H}_{2} \mathrm{CO}, \mathrm{CH}_{3} \mathrm{OH}, \mathrm{HC}_{3} \mathrm{~N}, \mathrm{CH}_{3} \mathrm{CN}$, and $c-\mathrm{C}_{3} \mathrm{H}_{2}$ are enhanced in the 

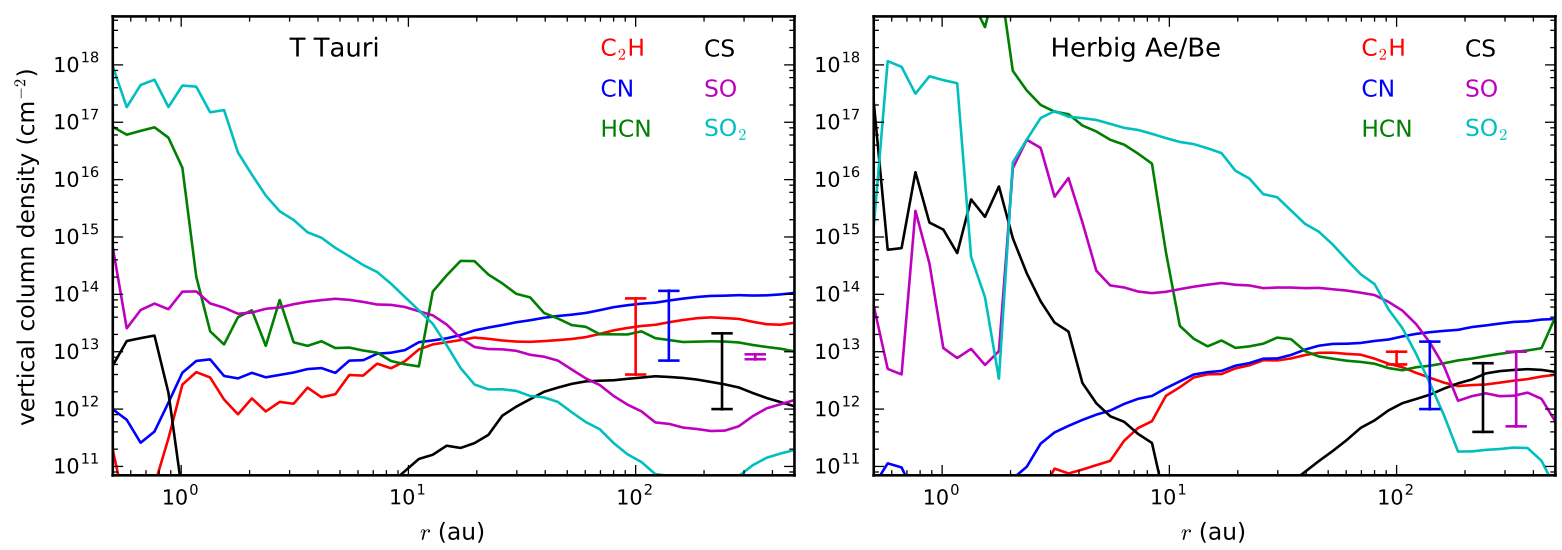

Fig. 8. Calculated vertical column densities (down to the midplane) of the radicals $\mathrm{C}_{2} \mathrm{H}$ and $\mathrm{CN}$ and some sulfur-bearing molecules as a function of radius $r$ for the T Tauri (left panel) and Herbig Ae/Be (right panel) disks. HCN is also shown to allow for the visualization of the $\mathrm{CN} / \mathrm{HCN}$ ratio. The ranges of column densities derived from observations are indicated by the vertical lines plotted in the outer disk (see Table 3 ).
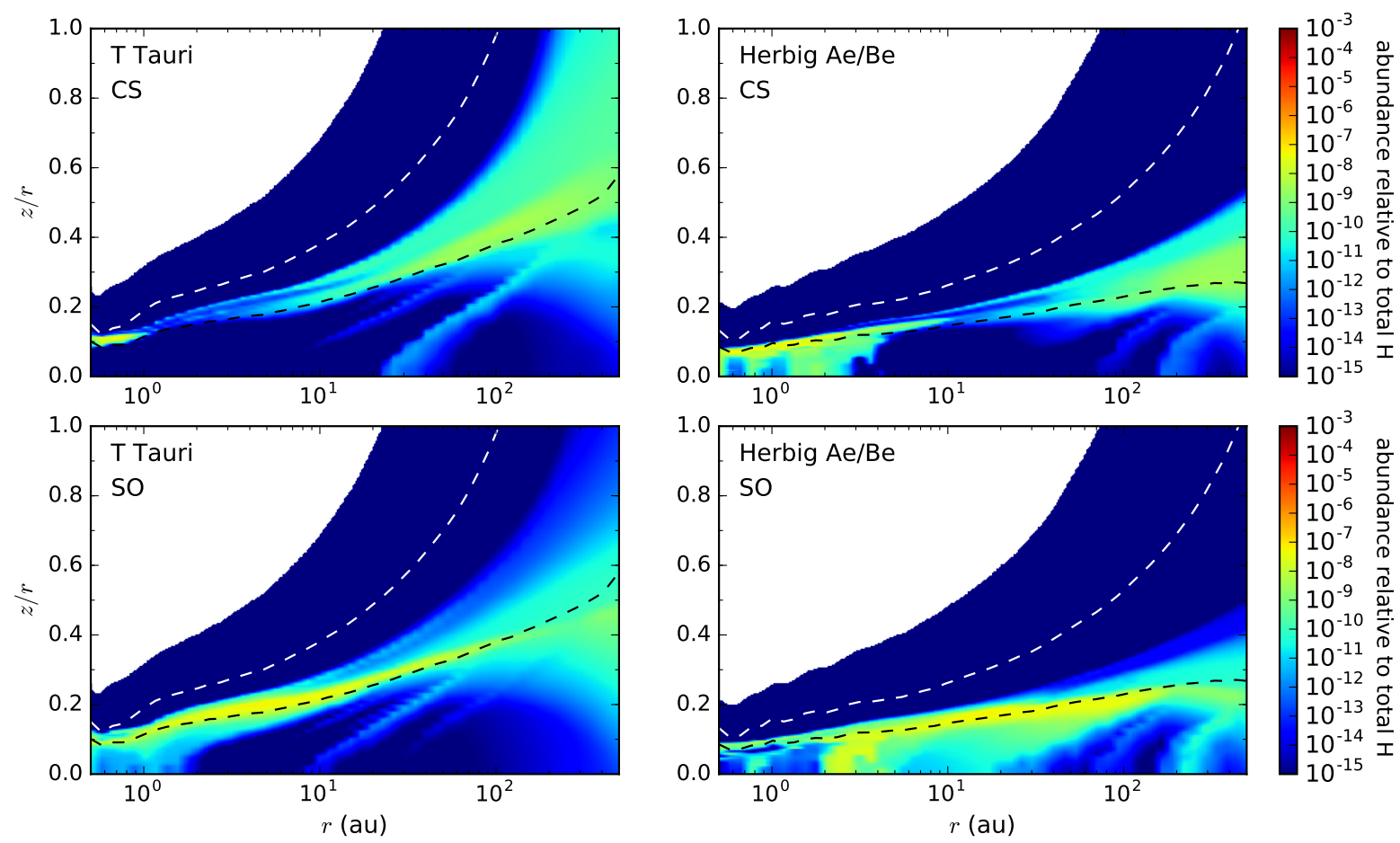

Fig. 9. As in Fig. 4 but for CS and SO.

warm inner regions (within a few au from the star), in particular in the Herbig Ae/Be disk, following the abundance enhancement of simple organics such as $\mathrm{C}_{2} \mathrm{H}_{2}$ and $\mathrm{HCN}$ (see Fig. 5). Such inner reservoir of complex organics, which is the result of hot gas-phase chemistry, could be detectable at millimeter wavelengths with ALMA provided the angular resolution and sensitivity are high enough.

\subsection{Sulfur-bearing molecules: CS and SO}

A couple of sulfur-bearing molecules, CS and SO, have been detected in protoplanetary disks. The observation of these species is interesting because it provides information on the degree of depletion of sulfur and because they can be used to probe phenomena, such as turbulence and dust traps (Guilloteau et al. 2012; Pacheco-Vázquez et al. 2016). CS was among the first molecules detected in disks (Dutrey et al. 1997) and since then has been observed in various T Tauri disks and in two Herbig Ae disks, while SO was first observed in the Herbig Ae disk AB Aur and was later observed in a couple of T Tauri disks (see references in Table 3). These observations were carried out at millimeter wavelengths and thus trace the outer regions of disks. Although the statistics of observed disks is small, it seems that both CS and SO have column densities of the order of $10^{12}-10^{13} \mathrm{~cm}^{-2}$ and that there is no substantial difference between T Tauri and Herbig Ae disks (see Table 3 ).

In our model, both CS and SO are mostly distributed in a layer located at intermediate heights (around $A_{V} \sim 1$ ), both in the T Tauri and the Herbig Ae/Be disks (see Fig. 9). In the inner regions (within a few au from the star) of the Herbig Ae/Be disk, these two molecules extend down to lower heights. The main formation pathways to both molecules involve various fast neutral-neutral reactions and thermal desorption from dust grains. The calculated column densities in the outer disk (beyond 
$100 \mathrm{au}$ ) are around $10^{12} \mathrm{~cm}^{-2}$ in both the TTauri and the Herbig Ae/Be disks, in line with the values derived from observations (see Fig. 8). The S-bearing molecule $\mathrm{SO}_{2}$ has not been detected in disks but it is predicted to have column densities of the same order as or slightly lower than SO in the outer disk (see Fig. 8). In the inner regions, the column densities of $\mathrm{CS}, \mathrm{SO}$, and $\mathrm{SO}_{2}$ are enhanced by various orders of magnitude, especially in the case of the Herbig Ae/Be disk, although these regions are likely to be strongly spatially diluted in the millimeter observations.

The column densities of CS and SO calculated here for the Herbig Ae/Be disk are similar to those obtained in the chemical model of the AB Aur disk presented by Fuente et al. (2010) and Pacheco-Vázquez et al. (2015). The main difference is that those models predict an enhancement in the column densities of CS and SO in the 100-200 au region, something that is not seen in the Herbig Ae/Be disk model presented here. The main reason of such difference is that those models assumed a more simplistic initial composition, with all the sulfur being initially in the form of CS, while in the present model we adopt a more realistic initial composition in which sulfur is initially distributed in various forms, mostly as S, $\mathrm{S}^{+}, \mathrm{CS}$, and CS ice (see Sect. 2.2). The choice of the initial composition can have non-negligible effects on the calculated abundances at ages typical of protoplanetary disks, something that we plan to investigate in detail in the future.

\subsection{Molecular ions}

The molecular ion $\mathrm{HCO}^{+}$is probably one of the most widely observed species in protoplanetary disks (see references in Table 3). In fact, it is remarkable that the detection rate of $\mathrm{HCO}^{+}$ is $100 \%$ in all the T Tauri and Herbig Ae disks targeted by Öberg et al. $(2010,2011)$ and by Guilloteau et al. (2016). Observed column densities are of the order of $10^{12} \mathrm{~cm}^{-3}$, with no significant difference between T Tauri and Herbig Ae disks (see Table 3). In our model, $\mathrm{HCO}^{+}$is mainly present in the outer disk at intermediate heights (see Fig. 10), where it is mainly formed by the reaction between $\mathrm{H}_{3}^{+}$and $\mathrm{CO}$. The calculated vertical column density in the outer disk is in the range $10^{12}-10^{13} \mathrm{~cm}^{-2}$ in both the T Tauri and the Herbig Ae/Be disks, in good agreement with the values derived from observations (see Fig. 11) and in line with results from previous chemical models of T Tauri disks (e.g., Aikawa \& Herbst 1999b; Aikawa et al. 2002; van Zadelhoff et al. 2003; Walsh et al. 2010, 2012; Semenov et al. 2011). Unlike in the Herbig Ae/Be disk model, in the T Tauri disk model the column density of $\mathrm{HCO}^{+}$(and other molecular ions) experience a decline beyond $\sim 200$ au. In these outer regions, the upper layers are more exposed to stellar FUV photons due to the flared shape of the disk, and atomic ions are favored at the expense of polyatomic ions, such as $\mathrm{HCO}^{+}$. This effect is probably a consequence of the particular geometry of the disk model adopted here and may not be a general characteristic of T Tauri disks. Therefore, according to the model, there is no reason to expect significantly different $\mathrm{HCO}^{+}$column densities in T Tauri and Herbig Ae disks.

Molecular ions other than $\mathrm{HCO}^{+}$are difficult to observe in protoplanetary disks. Nevertheless, sensitive observations with (sub-)millimeter interferometers have enabled the detection of $\mathrm{N}_{2} \mathrm{H}^{+}$in a few T Tauri disks and one Herbig Ae disk, HD 163296 (see references in Table 3). Derived column densities are highly dependent on each source and on each particular study. For example, in the disk around LkCa 15, Qi et al. (2003) derive $N\left(\mathrm{~N}_{2} \mathrm{H}^{+}\right)=3.1 \times 10^{13} \mathrm{~cm}^{-2}$ from observations with the OVRO array, while Dutrey et al. (2007) find a column density around
100 times smaller using IRAM PdBI. The chemistry of $\mathrm{N}_{2} \mathrm{H}^{+}$ is relatively simple as it is formed by the reaction between $\mathrm{H}_{3}^{+}$ and $\mathrm{N}_{2}$, which is favored in the coldest regions where $\mathrm{CO}$ is mostly in the form of ice, that is, in the outer midplane region. In our particular disk models, $\mathrm{CO}$ ice is present beyond a few tens of astronomical units in the TTauri disk, while in the Herbig Ae/Be disk, due to the much warmer dust temperatures, it is barely formed just in the outer edge (see Sect. 3.7). As a consequence, $\mathrm{N}_{2} \mathrm{H}^{+}$is present in the outer T Tauri disk with a vertical column density of $\sim 10^{11} \mathrm{~cm}^{-2}$ while it is almost absent in the Herbig Ae/Be disk (see Fig. 11). The vertical column densities calculated for $\mathrm{N}_{2} \mathrm{H}^{+}$are in line with the low range of observed values in the case of the T Tauri disk, while in the Herbig Ae/Be disk, $N\left(\mathrm{~N}_{2} \mathrm{H}^{+}\right)$is lower than observed in HD 163296 by more than one order of magnitude (see Fig. 11). The model also predicts that $\mathrm{N}_{2} \mathrm{H}^{+}$must have a ring-like distribution with an inner gap, something that has been verified observationally in the TW Hya disk (Qi et al. 2013c). The inner radius and vertical column density calculated for $\mathrm{N}_{2} \mathrm{H}^{+}$are highly dependent on which is the dust temperature structure across the disk, which in turn depends on parameters such as the stellar luminosity, the size and optical properties of dust grains, and the disk geometry (flared vs. flat). In any case, since disks around Herbig Ae/Be stars are expected to be significantly warmer than around T Tauri stars, one should expect larger amounts of $\mathrm{N}_{2} \mathrm{H}^{+}$in T Tauri disks than in Herbig Ae/Be disks.

Some deuterated ions, mostly $\mathrm{DCO}^{+}$but also $\mathrm{H}_{2} \mathrm{D}^{+}$and $\mathrm{N}_{2} \mathrm{D}^{+}$, have been observed at (sub-)millimeter wavelengths in a few protoplanetary disks (van Dishoeck et al. 2003; Ceccarelli et al. 2004; Guilloteau et al. 2006; Qi et al. 2008; Öberg et al. 2010, 2011; Mathews et al. 2013; Huang \& Öberg 2015; Teague et al. 2015; Huang et al. 2017). Here we do not specifically model deuterium chemistry (see, e.g., Willacy 2007; Teague et al. 2015), but we point out that these molecular ions are usually present in the cool outer disk and are useful to probe the fractional ionization. Estimates of the ionization fraction from observations of $\mathrm{H}_{2} \mathrm{D}^{+}$(which is present in the midplane of the cold outer disk) are a few times $10^{-10}$ (Ceccarelli et al. 2004), while values inferred from observations of $\mathrm{DCO}^{+}$(which is expected in upper layers) are as high as $\sim 10^{-7}$ (Qi et al. 2008; Teague et al. 2015). In our T Tauri and Herbig Ae/Be disk models, the ionization fraction in the midplane ranges from $\sim 10^{-10}$ in the denser inner regions to $\sim 10^{-8}$ in the outer disk (see Fig. 10). In these midplane regions, the fractional ionization is controlled by the cosmic-ray ionization rate and also depends on the gas density. As one moves to upper layers, where the gas is less dense, warmer, and less shielded against interstellar and stellar FUV photons, the ionization fraction increases gradually up to very high values in the surface of the disk (see Fig. 10). According to the model, there are no substantive differences in the ionization degree of disks around T Tauri and Herbig Ae/Be stars.

The ion $\mathrm{CH}^{+}$has been detected in a couple of disks, around the Herbig Be star HD 100546 and the Herbig Ae star HD 97048, using Herschel (Thi et al. 2011; Fedele et al. 2013). The analysis of the emission lines in HD 100546 indicates that $\mathrm{CH}^{+}$has an excitation temperature of 100-300 K, with most emission arising from regions inside $100 \mathrm{au}$. In our model, $\mathrm{CH}^{+}$is distributed along a surface layer on top of $\mathrm{HCO}^{+}$(see Fig. 10), where it is mainly formed by the reaction of $\mathrm{C}^{+}$with hot $\mathrm{H}_{2}$ and FUV-pumped vibrationally excited $\mathrm{H}_{2}$ (Agúndez et al. 2010) and reaches a maximum fractional abundance of $\sim 10^{-7}$. The calculated distribution of $\mathrm{CH}^{+}$is very similar in the T Tauri and 

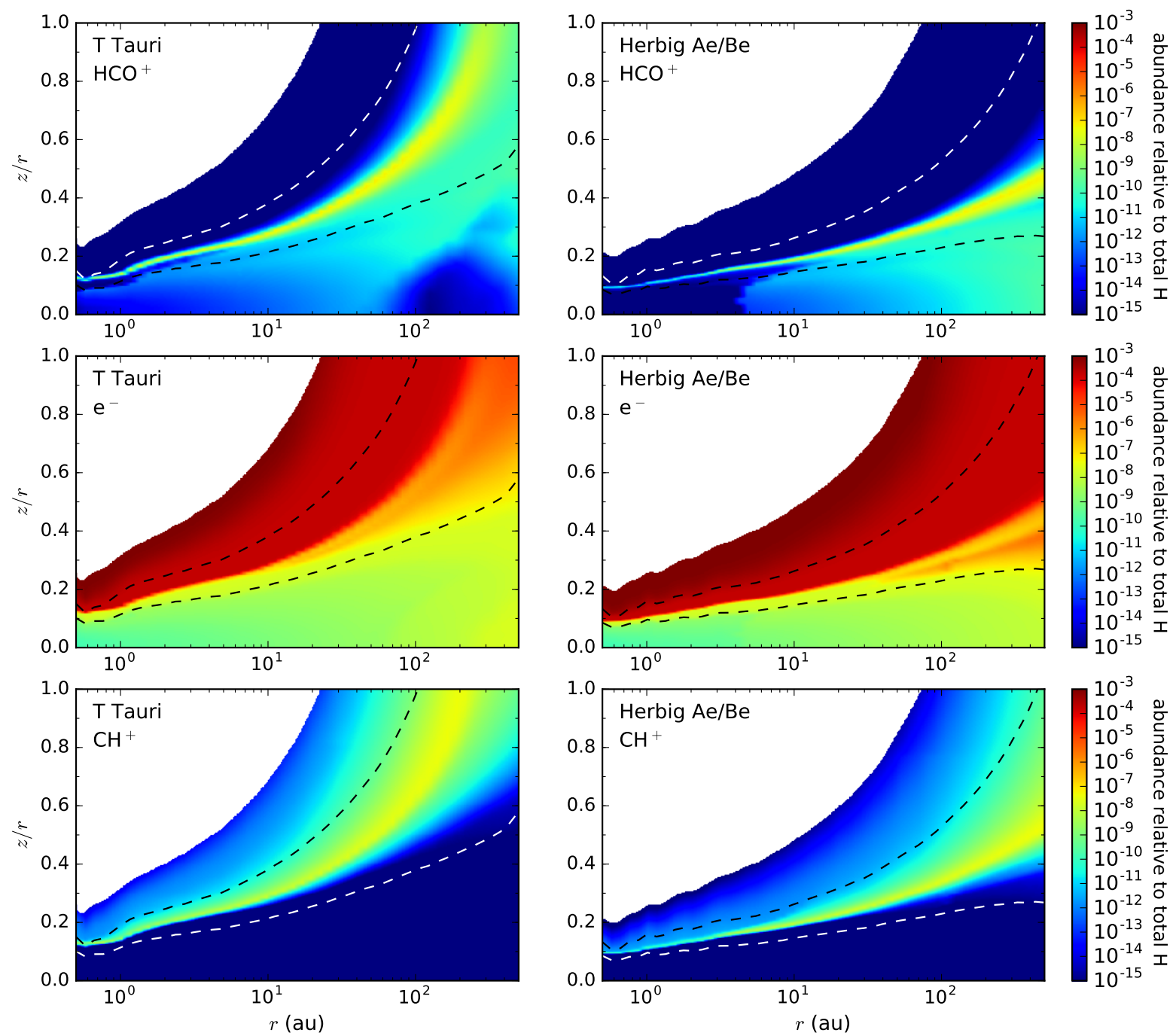

Fig. 10. As in Fig. 4 but for $\mathrm{HCO}^{+}$, electrons, and $\mathrm{CH}^{+}$.
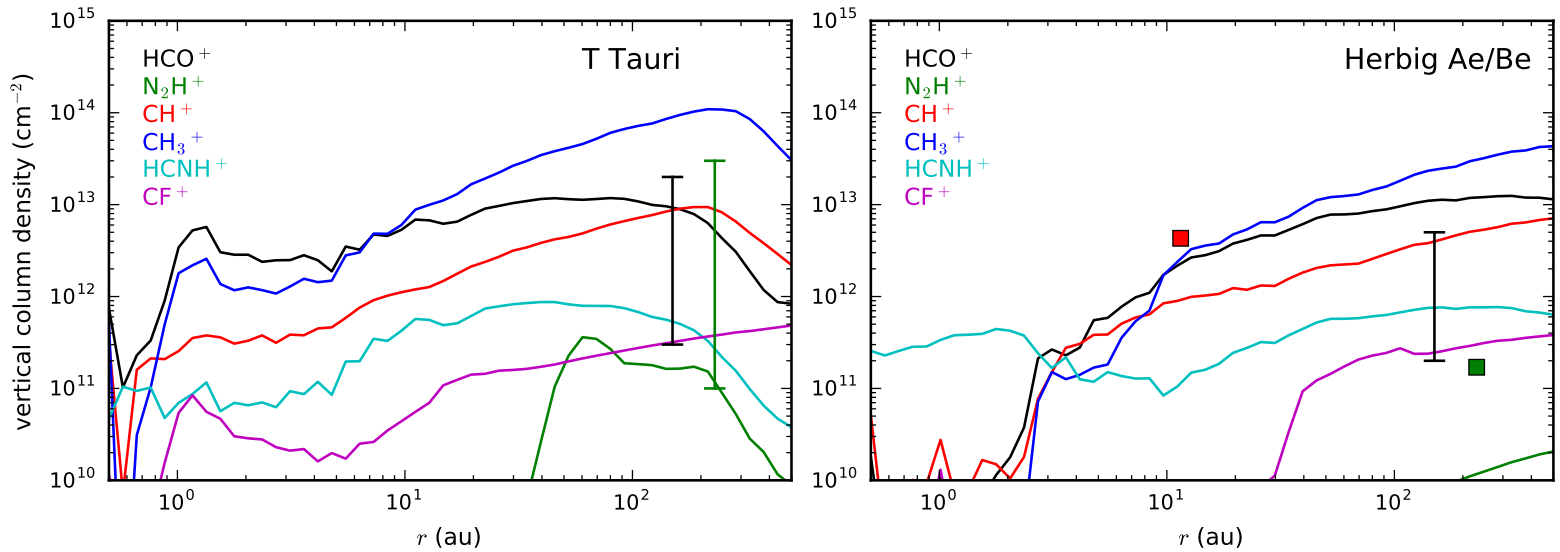

Fig. 11. Calculated vertical column densities (down to the midplane) of selected molecular ions as a function of radius $r$ for the T Tauri (left panel) and Herbig Ae/Be (right panel) disks. Column densities derived from observations are indicated by vertical lines or squares, with their radial locations corresponding to the approximate region probed by observations (see Table 3).

Herbig Ae/Be disks. The model predicts that the largest column densities are reached in the outer disk (>100 au; see Fig. 11) contrary to what observations of HD 100546 indicate. We, however, note that $\mathrm{CH}^{+}$emission is probably very sensitive to the temperature structure and geometry of each particular disk. In fact, $\mathrm{CH}^{+}$ has only been detected in a couple of disks from the sample of 22 Herbig Ae/Be and 8 T Tauri systems surveyed by Fedele et al. (2013).

Apart from $\mathrm{HCO}^{+}$, the most abundant molecular ions in the disk are $\mathrm{H}_{3}^{+}, \mathrm{CH}_{2}^{+}, \mathrm{CH}_{3}^{+}$(see Fig. 11), and $\mathrm{C}_{2} \mathrm{H}_{2}^{+}$, species which 
are very difficult to detect. While $\mathrm{H}_{3}^{+}$is mostly present in the midplane of the outer disk, the hydrocarbon cations are essentially present in the disk surface. For example, the formation of $\mathrm{CH}_{2}^{+}$and $\mathrm{CH}_{3}^{+}$is strongly linked to that of $\mathrm{CH}^{+}$(Agúndez et al. 2010). Molecular ions not yet observed in disks but potentially detectable are $\mathrm{HCNH}^{+}$(the precursor of $\mathrm{HCN}$ and $\mathrm{HNC}$ in the outer disk) and $\mathrm{CF}^{+}$(formed in the disk PDR). Both ions are polar and have column densities in excess of $10^{11} \mathrm{~cm}^{-2}$ (see Fig. 11), although detecting them may be challenging because their dipole moments are not very high. An additional interesting feature emerging from the model is that most of the positive charge is not carried out by molecular ions but by atomic ions (metals in the disk midplane and carbon in the disk surface).

\subsection{Ices and snow lines}

Ices account for an important percentage of the matter of protoplanetary disks. The high densities and cold temperatures prevailing in the midplane regions ensure a rapid and efficient adsorption of gas-phase molecules onto dust grains, where they settle and live long in the form of ice. The presence or absence of a particular type of ice in a certain disk region depends on the balance between adsorption and desorption, and, because adsorption rates are similar for most molecules (see Sect. 2.2.5), the extent of each particular type of ice is determined by its specific desorption rate. According to the model, among the various desorption mechanisms considered (see Sect. 2.2.6), thermal desorption is clearly the most important and the one that shapes the bulk distribution of most ices. We however note that for those species with large binding energies, the vertical extent of ices in the outer disk is essentially controlled by photodesorption by interstellar and stellar FUV radiation. In these outer regions, dust temperatures may not be high enough to trigger thermal desorption of strongly bonded ices, while FUV photons can penetrate down to intermediate heights and provide an efficient means of desorbing ice molecules. The main effect of photodesorption is that it shifts down the snow line ${ }^{13}$ of highly polar molecules in the outer disk, enhancing their gas-phase abundance at intermediate heights. In summary, the distribution of ices with low binding energies, such as $\mathrm{CO}$ and $\mathrm{N}_{2}$, is controlled by thermal desorption, while for ices with large binding energies, like $\mathrm{NH}_{3}$ and $\mathrm{H}_{2} \mathrm{O}$ (see Table 2), distribution is determined by thermal desorption in the inner regions and by photodesorption in the outer disk. Other desorption mechanisms, such as cosmic-ray induced desorption or photodesorption by FUV photons generated through the Prasad-Tarafdar mechanism, are much less important.

In protoplanetary disks, ices are mostly distributed around the midplane, from the very outer disk down to an inner edge, which is given by the location of the snow line in the midplane. Since the midplane snow line is essentially controlled by thermal desorption (and thus by the binding energy of the particular ice and by the dust temperature structure of the disk along the midplane), ices appear progressively in the radial direction according to their binding energies. In Fig. 12, we show the calculated distributions of $\mathrm{CO}$ and $\mathrm{H}_{2} \mathrm{O}$ ices in the T Tauri and Herbig Ae/Be disks. First focusing on the T Tauri disk model, we see that $\mathrm{CO}$ ice, for which the adopted binding energy is $1575 \mathrm{~K}$, is only present beyond a few tens of astronomical units, while $\mathrm{H}_{2} \mathrm{O}$ ice, which has a much higher binding energy (the adopted value is $5773 \mathrm{~K}$ ), is present all over the disk midplane. The fact that

\footnotetext{
13 The snow line of a particular species is defined here as the transition region where its gas and ice abundances become equal.
}

water ice extends down to the inner disk edge is a particular outcome of the T Tauri disk model adopted here, which results in dust that is too cold to allow for efficient thermal desorption of water ice in the inner disk midplane. Since dust temperatures are strongly dependent on parameters such as the size of dust grains, other T Tauri disk models may find water snow lines that are further out compared to that obtained here (see, e.g., Walsh et al. 2015). An obvious difference between the T Tauri and the Herbig Ae/Be disk models is that in the latter, snow lines shift to larger radii owing to the warmer dust temperatures (see Fig. 12), and as a consequence, the mass of ices in the disk becomes smaller.

The abundance distributions of $\mathrm{CO}$ and $\mathrm{H}_{2} \mathrm{O}$ ices shown in Fig. 12 serve to illustrate two extreme cases of molecules with low and high binding energies, respectively (see Table 2). Other ices with binding energies between those of $\mathrm{CO}$ and $\mathrm{H}_{2} \mathrm{O}$ have intermediate distributions between those of these two molecules. In general, the smaller the binding energy, the larger the radius to which the snow line shifts. This relationship is illustrated in Fig. 13, where we plot the radius at which the midplane snow line lies for various ices as a function of their binding energies. There is a clear trend which indicates that both quantities are related by a power law of the type $E_{D} \propto r_{\mathrm{ms}}^{-q}$, where $r_{\mathrm{ms}}$ stands for radius of midplane snow line. A fit to the data points in Fig. 13 yields an exponent $q$ of $\sim 0.23$ in both the T Tauri and the Herbig Ae/Be disks. This behavior is not surprising since the midplane snow line of a given ice is essentially located at the radius at which the dust temperature in the midplane becomes similar to the condensation temperature of the ice, and the latter is directly proportional to the binding energy of the ice. That is, the observed relation between binding energy and midplane snow line merely reflects how the midplane dust temperature varies with radius. In Fig. 13 we have overplotted as a solid line the radial profile of the dust temperature in the midplane scaled up by a factor of 45 . The comparison between solid line and data points in Fig. 13 suggests that a factor of proportionality between binding energy and condensation temperature in the range 30-50 is adequate for the protoplanetary disks modeled here. For comparison, Hollenbach et al. (2009) calculate this factor of proportionality to be $\sim 50$ while Martín-Doménech et al. (2014) quote a value of 30 (from Attard \& Barnes 1998).

Using observations to reveal the location of snow lines in protoplanetary disks is still challenging, although ALMA observations are starting to permit interesting constraints. For example, in the disk around the T Tauri star TW Hya the CO snow line has been found to lie at a radius of $\sim 20$ au (Schwarz et al. 2016; Zhang et al. 2017), while in the disk around the Herbig Ae star HD 163296 it has been located at a radius of 90 au from the star (Qi et al. 2015). These two observational points are included in Fig. 13. While the CO snow line derived for TW Hya is close to the value calculated in our T Tauri disk model, the radius derived for HD 163296 is much smaller than indicated by our Herbig $\mathrm{Ae} / \mathrm{Be}$ disk model, which puts the $\mathrm{CO}$ snow line even beyond the outer disk edge. We however note that the coincidence in the case of TW Hya is very likely accidental because our generic T Tauri disk model is not aimed to represent either the TW Hya disk or any other particular disk. The observational finding of a CO snow line farther out in the HD 163296 disk than in the TW Hya disk is in line with the general expectation that snow lines are shifted to larger radii in Herbig Ae/Be disks compared to T Tauri disks, although taking into account the poor statistics, consisting of just one object of each type, this result may be fortuitous. Expanding the sample of disks seems mandatory to draw more definitive conclusions. It will also be very interesting 

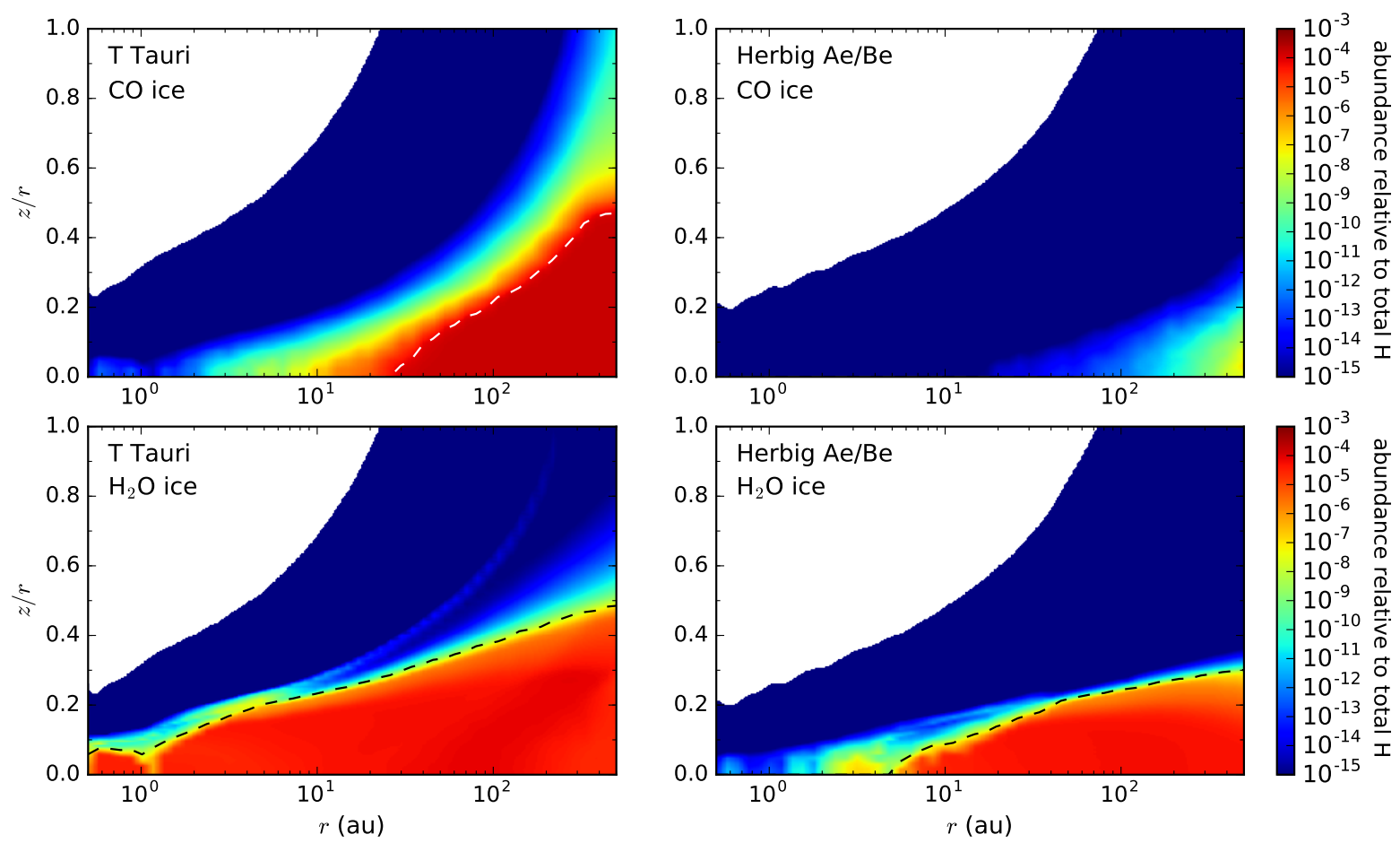

Fig. 12. Calculated distributions of $\mathrm{CO}$ and $\mathrm{H}_{2} \mathrm{O}$ ices as a function of radius $r$ and height over radius $z / r$ for the T Tauri (left panel) and Herbig Ae/Be (right panel) disks. The dashed line in each panel indicates the location of the corresponding snow line.

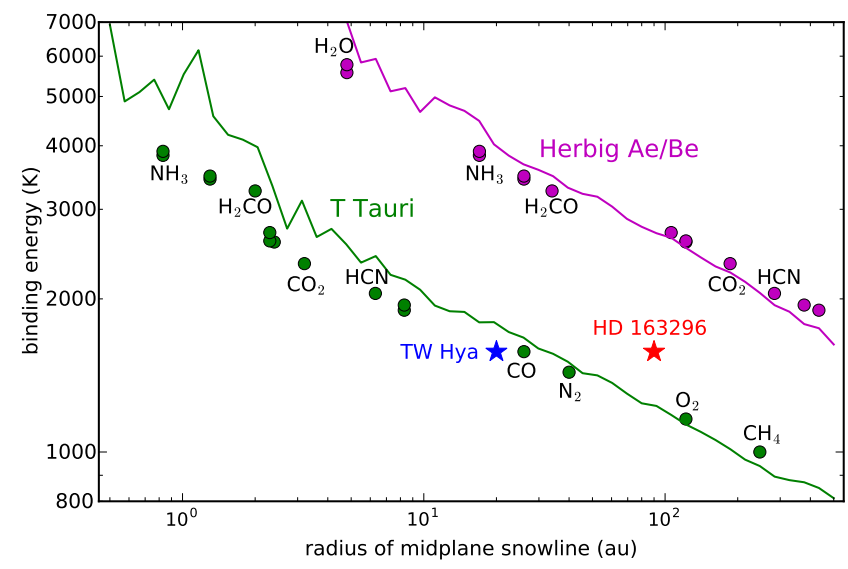

Fig. 13. Midplane snow line of various ices (along $x$-axis) as a function of the binding energy (along $y$-axis) in the T Tauri (green circles) and Herbig $\mathrm{Ae} / \mathrm{Be}$ (magenta circles) disks. Observational $\mathrm{CO}$ snow lines in TW Hya (Schwarz et al. 2016; Zhang et al. 2017) and HD 163296 (Qi et al. 2015) are also shown. We also plot the dust temperature in the midplane (scaled up by a factor of 45) as a function of radius in the T Tauri (green solid line) and Herbig Ae/Be (magenta solid line) disk models.

to observationally locate the snow line of different molecules in the same disk, so that the sequence of snow lines of ices with different binding energies depicted in Fig. 13 can be tracked.

\section{Influence of the stellar spectrum and the photodestruction rates}

Chemical models of protoplanetary disks contain so many ingredients to deal with the multiple processes at work that the output abundance distributions can be sensitive to many of them. In this study we put the focus on the photochemistry and thus here we evaluate the influence of a couple of related aspects: the spectrum of the star and the way in which photodestruction rates are computed. We concentrate on the T Tauri disk model for this sensitivity analysis.

T Tauri stars are cool, with effective temperatures of the order of $4000 \mathrm{~K}$, although they usually have an important FUV excess that can greatly affect the photochemistry of the disk. To evaluate the influence of this FUV excess we compare our fiducial T Tauri disk model, in which we consider the stellar spectrum of TW Hya, with a model in which we assume that the star emits as a blackbody at a temperature of $4000 \mathrm{~K}$ (both spectra are shown in Fig. 1). The lack of FUV excess in this latter case results in much lower photodestruction rates (compare our values in Table B.1 with those of van Dishoeck et al. 2006, or see Heays et al. 2017). The net effect is that without FUV excess, most photodestruction rates are dominated by the ISRF rather than by stellar radiation. As as example, in the left panel of Fig. 14 we show the photodissociation rate of $\mathrm{CO}$ as a function of height at a radius of $1 \mathrm{au}$, where both the contributions of the ISRF and the star are taken into account. It is seen that in the upper disk, where photodestruction rates are dominated by stellar photons (see Fig. 3), the photodissociation rate of $\mathrm{CO}$ in the $4000 \mathrm{~K}$ blackbody star model is below that in the fiducial model by many orders of magnitude. A similar behavior occurs for the photodestruction rates of other species. As a consequence, the $\mathrm{CO} / \mathrm{C} / \mathrm{C}^{+}$interface shifts to upper heights and the atomic carbon layer becomes wider (see right panels in Fig. 14). In general, the photochemically active layer, where for example the transition $\mathrm{H}_{2} \mathrm{O} / \mathrm{OH}$ is located and the radicals $\mathrm{C}_{2} \mathrm{H}$ and $\mathrm{CN}$ are, is shifted to upper layers. This overall shift of the photochemistry to upper, less dense, and warmer layers, together with the lower strength of the FUV radiation field, induce important changes in the abundances of several molecules. For example, stable 

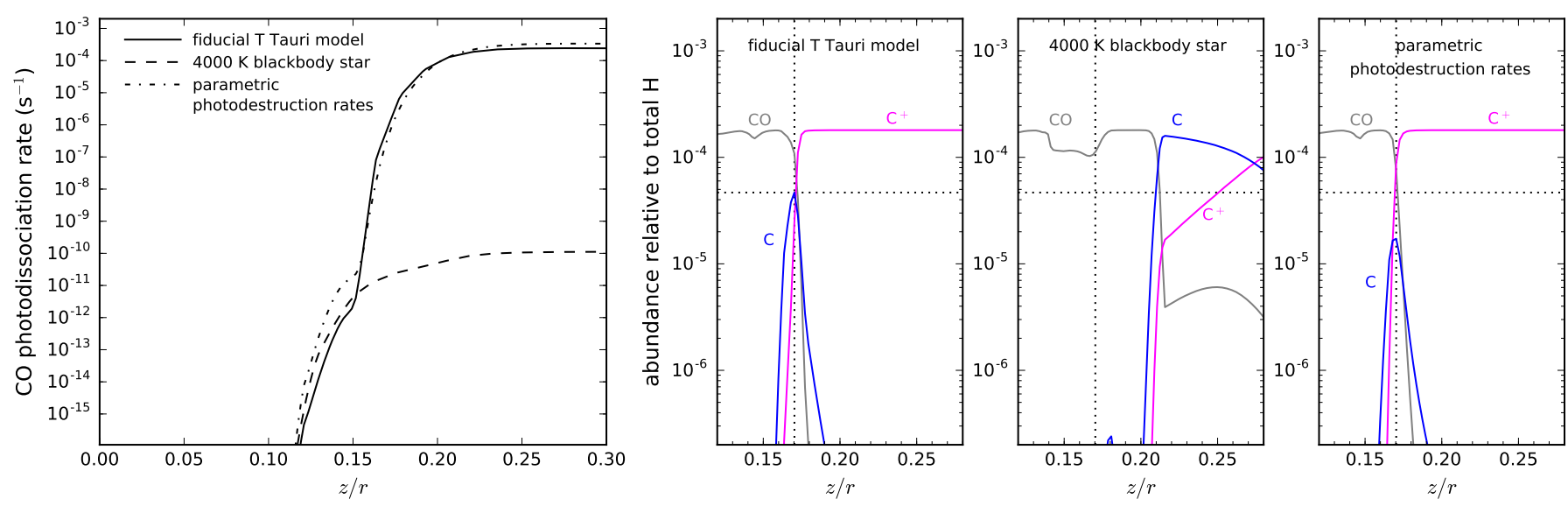

Fig. 14. Vertical structure of the $\mathrm{CO}$ photodissociation rate (left panel) and the $\mathrm{CO} / \mathrm{C} / \mathrm{C}^{+}$interface (right panels) at a radius of 1 au from the star for the T Tauri disk. We show results from our fiducial model, a model in which a $4000 \mathrm{~K}$ blackbody stellar spectrum is considered, and a model in which all photodestruction rates are computed using parametric expressions (see text). The dotted lines in the three right panels indicate the peak abundance of atomic carbon and the height at which it is reached in the fiducial model. The small drop in the abundance of CO seen around $z / r \sim 0.15$, which is more marked in the $4000 \mathrm{~K}$ blackbody star model, is due to a maximum in the abundance of water.
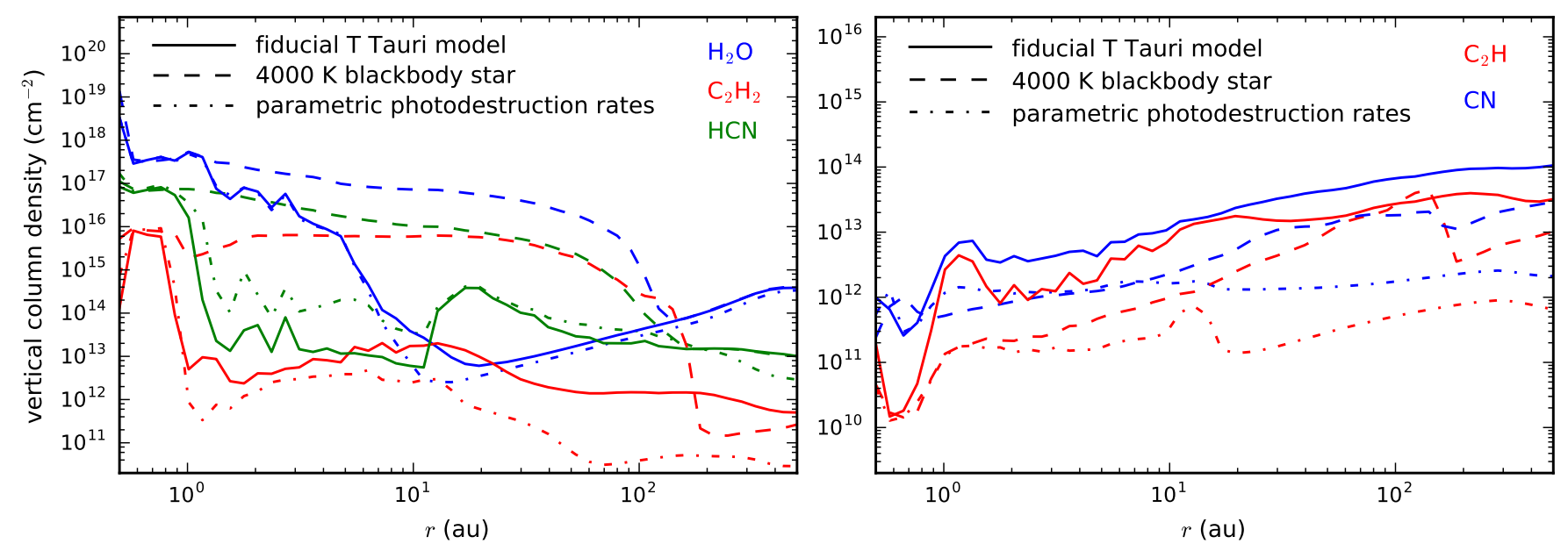

Fig. 15. Calculated vertical column densities down to the midplane of $\mathrm{H}_{2} \mathrm{O}, \mathrm{C}_{2} \mathrm{H}_{2}$, and $\mathrm{HCN}$ (left panel) and the radicals $\mathrm{C}_{2} \mathrm{H}$ and $\mathrm{CN}$ (right panel) as a function of radius $r$ for the T Tauri disk. We show results from our fiducial model, a model in which a $4000 \mathrm{~K}$ blackbody stellar spectrum is considered, and a model in which all photodestruction rates are computed using parametric expressions (see text).

molecules like water and the simple organics $\mathrm{C}_{2} \mathrm{H}_{2}$ and $\mathrm{HCN}$ become more abundant at intermediate disk radii compared to the fiducial model (see left panel in Fig. 15), while the radicals $\mathrm{C}_{2} \mathrm{H}$ and $\mathrm{CN}$ experience a decline in their abundances (see right panel in Fig. 15).

The way in which photodestruction rates are computed may also have an influence on the chemical structure of the photochemically active layer of disks (e.g., Walsh et al. 2012). In our fiducial model, we have used the Meudon PDR code to compute the photodissociation rates of $\mathrm{H}_{2}$ and $\mathrm{CO}$ by solving the excitation and the line-by-line FUV radiative transfer to properly account for self and mutual shielding effects and have computed photodestruction rates for a variety of species using the cross sections compiled in Appendix A. To investigate the effect of using a simpler approach, we have run a T Tauri disk model in which all photodestruction rates are computed through the parametric expression in Eq. (7). The $\alpha$ and $\gamma$ parameters are taken from Table B.1, while for $\mathrm{H}_{2}$ and $\mathrm{CO}$ we adopt unattenuated rates and dust shielding factors from Heays et al. (2017). In the left panel of Fig. 14 we compare the resulting
$\mathrm{CO}$ photodissociation rate as a function of height at a radius of 1 au given by this parametric photodestruction rates approach with that resulting from our fiducial model. It is seen that in the upper disk both approaches yield similar results, although at lower heights, where photodestruction is dominated by ISRF photons (see Fig. 3), the parametric approach overestimates the photodissociation rate of $\mathrm{CO}$. The resulting $\mathrm{CO} / \mathrm{C} / \mathrm{C}^{+}$interface is very similar in both scenarios, although it is worth noting that the peak abundance of atomic carbon is lower when the parametric photodestruction rates are used. This has some consequences for the abundances of carbon-bearing species typically formed under the action of photochemistry, such as the radicals $\mathrm{C}_{2} \mathrm{H}$ and $\mathrm{CN}$. In fact, these two radicals reach abundances significantly lower in the outer disk when parametric photodestruction rates are used compared to the fiducial model (see right panel in Fig. 15). Other species are also affected to different degrees. For example, while the column density of water does not change much, acetylene becomes less abundant and hydrogen cyanide is slightly enhanced in the range of radii between 1 and 10 au (see left panel in Fig. 15). 


\section{Conclusions}

We have developed a model aimed to compute the chemical composition of a generic protoplanetary disk around a young star. The model considers a passively irradiated disk in steady state and computes the physical and chemical structure of the disk with particular attention to the disk photochemistry. In particular we have compiled cross sections for 29 molecules and 8 atoms and computed the photodissociation and photoionization rates at each location in the disk by solving the FUV radiative transfer with the Meudon PDR code in a 1+1D approach.

We have applied the model to perform a comparative study of the chemistry of disks around low-mass (T Tauri) and intermediate-mass (Herbig $\mathrm{Ae} / \mathrm{Be}$ ) stars. Infrared and (sub-)millimeter observations of T Tauri and Herbig disks point to a lower detection rate of molecules in the latter type of disks and, for some species, somewhat lower abundances. Motivated by the observational studies, we have investigated the potential chemical differentiation between disks around these two types of stars, which have very different masses and spectra. We find that globally the chemical behavior of these two types of disk is quite similar, with some important differences driven by the higher stellar UV flux and the warmer temperatures of Herbig Ae/Be disks.

Water vapor and the simple organic molecules $\mathrm{C}_{2} \mathrm{H}_{2}, \mathrm{HCN}$, and $\mathrm{CH}_{4}$ are predicted to be very abundant $\left(\sim 10^{-4}\right.$ for $\mathrm{H}_{2} \mathrm{O}$ and a few $\times 10^{-5}$ for the organics) in the hot inner regions of disks around both T Tauri and Herbig Ae/Be stars. The main difference between the two types of disk is that these molecules extend over a larger region in Herbig Ae/Be disks due to the warmer temperatures attained, a finding that is in agreement with the models by Walsh et al. (2015) but in contrast with IR observations that find a much lower detection rate of water and simple organics toward Herbig Ae/Be disks than toward T Tauri disks. This latter fact is probably caused by observational aspects rather than by substantive differences in the chemistry between these two types of disk. For example, Antonellini et al. (2016) point out that Herbig stars are brighter than T Tauri stars resulting in a higher level of mid-IR continuum and smaller line/continuum ratios. If true, the higher sensitivity and spectral resolution of the James Webb Space Telescope with respect to Spitzer could allow to increase the detection rate of water and simple organics in Herbig Ae/Be systems.

Concerning the outer regions of disks, observations point to a lower detection rate of molecules and to somewhat lower abundances in Herbig Ae disks compared to T Tauri disks, although the statistics of Herbig Ae objects observed is small. Our model indicates that in general there are not drastic differences between T Tauri and Herbig Ae disks concerning the abundances of molecules typically observed in disks at (sub-)millimeter wavelengths. More specifically, various molecules, such as $\mathrm{H}_{2} \mathrm{CO}$, $\mathrm{CS}, \mathrm{SO}$, and $\mathrm{HCO}^{+}$, are observed with similar abundances in the outer regions of T Tauri and Herbig Ae disks and the model satisfactorily finds no clear differentiation between both types of disks for these species. For other species, such as HCN and the radicals $\mathrm{C}_{2} \mathrm{H}$ and $\mathrm{CN}$, observations suggest that T Tauri disks may retain somewhat larger abundances than Herbig Ae disks in the outer regions, while the model indeed predicts that these species are slightly less abundant in Herbig Ae disks than in T Tauri disks. In the case of the radicals $\mathrm{C}_{2} \mathrm{H}$ and $\mathrm{CN}$, which are produced by the action of photochemistry, the slightly lower abundances calculated in Herbig Ae disks are caused by the higher UV flux, which narrows the photochemically active layer and limits the ability of photochemistry to synthesize molecules.
In any case, for $\mathrm{HCN}, \mathrm{C}_{2} \mathrm{H}$, and $\mathrm{CN}$, the observed and calculated abundance differences between both types of disks are small, of a factor of a few at most.

For other organic molecules with a certain complexity such as $\mathrm{CH}_{3} \mathrm{OH}, \mathrm{HC}_{3} \mathrm{~N}, \mathrm{CH}_{3} \mathrm{CN}$, and $c-\mathrm{C}_{3} \mathrm{H}_{2}$, the match with observed values is not satisfactory. This fact, together with the ample dispersion of abundances calculated by different chemical models in the literature, points to the chemistry of these molecules not yet being as robust as for simpler species. In addition, grain-surface chemistry, not included in our model, is likely playing an active role in the synthesis of some of these molecules.

A clear differentiation between T Tauri and Herbig Ae disks is found concerning ices. The warmer temperatures of Herbig Ae disks shift snow lines to larger radii compared to T Tauri disks and as a consequence disks around intermediate-mass stars are expected to contain a substantially lower mass of ices compared to T Tauri disks.

Acknowledgements. We thank the anonymous referee for a detailed reading and for a constructive report which helped to improve this manuscript. M.A. acknowledges funding support from the European Community 7th Framework Programme through a Marie Curie Intra-European Individual Fellowship (grant 235753), from the European Research Council through ERC Synergy grant 610256, and from Spanish MINECO through the Ramón y Cajal programme (RyC-2014-16277) and grant AYA2016-75066-C2-1-P. This work was partly supported by the CNRS program Physique et Chimie du Milieu Interstellaire (PCMI) co-funded by the Centre National d'Études Spatiales (CNES). We thank J. R. Goicoechea, E. Bron, S. Cazaux, G. M. Muñoz-Caro, R. Martín-Doménech, and A. Fuente for useful discussions, S. Miyake for help in the treatment of cross sections from the former version of the Leiden database, and E. A. Bergin, C. E. Brion, J. A. Nuth, D. M. P. Holland, K.-L. Han, and J.-H. Fillion for kindly providing useful data.

\section{References}

Aguado, A., Roncero, O., Zanchet, A., et al. 2017, ApJ, 838, 33

Agúndez, M., \& Cernicharo, J. 2006, ApJ, 650, 374

Agúndez, M., \& Wakelam, V. 2013, Chem. Rev., 113, 8710

Agúndez, M., Cernicharo, J., \& Goicoechea, J. R. 2008, A\&A, 483, 831

Agúndez, M., Goicoechea, J. R., Cernicharo, J., et al. 2010, ApJ, 713, 662

Aikawa, Y. 2007, ApJ, 656, L93

Aikawa, Y., \& Herbst, E. 1999a, ApJ, 526, 314

Aikawa, Y., \& Herbst, E. 1999b, A\&A, 351, 233

Aikawa, Y., \& Herbst, E. 2001, A\&A, 371, 1107

Aikawa, Y., \& Nomura, H. 2006, ApJ, 642, 1152

Aikawa, Y., Miyama, S. M., Nakano, T., \& Umebayashi, T. 1996, ApJ, 467, 684 Aikawa, Y., Umebayashi, T., Nakano, T., \& Miyama, S. M. 1997, ApJ, 486, L51 Aikawa, Y., Umebayashi, T., Nakano, T., \& Miyama, S. M. 1999, ApJ, 519, 705 Aikawa, Y., van Zadelhoff, G. J., van Dishoeck, E. F., \& Herbst, E. 2002, A\&A, 622,632

Aikawa, Y., Momose, M., Thi, W.-F., et al. 2003, PASJ, 55, 11

Akimkin, V., Zhukovska, S., Wiebe, D., et al. 2013, ApJ, 766, 8

Albertsson, T., Semenov, D., \& Henning, Th. 2014, ApJ, 784, 39

Andersson, S., \& van Dishoeck, E. F. 2008, A\&A, 491, 907

Anicich, V. G. 2003, JPL Publication 03-19

Antonellini, S., Kamp, I., Lahuis, F., et al. 2016, A\&A, 585, A61

Apaydin, G., Fink, W. H., \& Jackson, W. M. 2004, J. Chem. Phys., 121, 9368

Aresu, G., Kamp, I., Meijerink, R., et al. 2011, A\&A, 526, A163

Arthur, N. L. 1986, J. Chem. Soc., Faraday Trans. 2, 82, 331

Attard, G., \& Barnes, C. 1998, Surfaces (Oxford Science Publications), 72

Atkinson, R., Baulch, D. L., Cox, R. A., et al. 2004, Atm. Chem. Phys., 4, 1461

Atkinson, R., Baulch, D. L., Cox, R. A., et al. 2006, Atm. Chem. Phys., 6, 3625

Au, J. W., Cooper, G., Burton, G. R., et al. 1993, Chem. Phys., 173, 209

Ayres, T. R. 2010, ApJS, 187, 149

Banzatti, A., Pontoppidan, K. M., Salyk, C., et al. 2017, ApJ, 834, 152

Barsuhn, J., \& Nesbet, R. K. 1978, J. Chem. Phys., 68, 2783

Bast, J. E., Lahuis, F., van Dishoeck, E. F., \& Tielens, A. G. G. M. 2013, A\&A, 551, A118

Baulch, D. L., Bowman, C. T., Cobos, C. J., et al. 2005, J. Phys. Chem. Ref. Data, 34,757

Bergin, E., Calvet, N., D’Alessio, P., \& Herczeg, G. J. 2003, ApJ, 591, L159 
Bergin, E. A., Aikawa, Y., Blake, G. A., \& van Dishoeck, E. F. 2007, in Protostars and Planets V, eds. B. Reipurth, D. Jewitt, \& K. Keil (University Arizona Press), 751

Bergin, E. A., Hogerheijde, M. R., Brinch, C., et al. 2010, A\&A, 521, L33

Bergin, E. A., Du, F., Cleeves, L. I., et al. 2016, ApJ, 831, 101

Bertin, M., Fayolle, E. C., Romanzin, C., et al. 2012, Phys. Chem. Chem. Phys., 14, 9929

Bertin, M., Fayolle, E. C., Romanzin, C., et al. 2013, ApJ, 779, 120

Bertin, M., Romanzin, C., Doronin, M., et al. 2016, ApJ, 817, L12

Bethell, T., \& Bergin, E. 2009, Science, 326, 1675

Biehl, H., Schönnenbeck, G., Stuhl, F., \& Staemmler, V. 1994, J. Chem. Phys., 101,3819

Blevins, S. M., Pontoppidan, K. M., Banzatti, A., et al. 2016, ApJ, 818, 22

Brion, C. E., Dyck, M., \& Cooper, G. 2005, J. Electron Spectrosc. Relat. Phenom., 144, 127

Bron, E., Le Bourlot, J., \& Le Petit, F. 2014, A\&A, 569, A100

Bruna, P. J., \& Hirsch, G. 1987, Mol. Phys., 61, 1359

Bruna, P. J., Kammer, W. E., \& Vasudevan, K. 1975, Chem. Phys., 9, 91

Burcat, A., \& Ruscic, B. 2005, ANL-05/20 and TAE 960 Technion-IIT

Burton, G. R., Chan, W. F., Cooper, G., \& Brion, C. E. 1993, Chem. Phys., 177, 217

Burke, D. J., \& Brown, W. A. 2010, Phys. Chem. Chem. Phys., 12, 5947

Callear, A. B., \& Metcalfe, P. 1976, Chem. Phys., 14, 275

Cameron, M., Sivakumaran, V., Dillon, T. J., \& Crowley, J. N. 2002, Phys. Chem. Chem. Phys., 4, 3628

Carney, M. T., Hogerheijde, M. R., \& Loomis, R. A., 2017, A\&A, 605, A21

Carr, J. S., \& Najita, J. R. 2008, Science, 319, 1504

Carr, J. S., \& Najita, J. R. 2011, ApJ, 733, 102

Carr, J. S., \& Najita, J. R. 2014, ApJ, 788, 66

Carr, J. S., Tokunaga, A. T., \& Najita, J. 2004, ApJ, 603, 213

Castelli, F., \& Kurucz, R. L. 2004, ArXiv e-prints [arXiv: astro-ph/0405087]

Cazaux, S., \& Tielens, A. G. G. M. 2002a, ApJ, 575, L29

Cazaux, S., \& Tielens, A. G. G. M. 2002b, ApJ, 577, L127

Ceccarelli, C., Dominik, C., Lefloch, B., et al. 2004, ApJ, 607, L51

Cernicharo, J. 2004, ApJ, 608, L41

Chaabouni, H., Bergeron, H., Baouche, S., et al. 2012, A\&A, 538, A128

Chan, W. F., Cooper, G., \& Brion, C. E. 1993a, Chem. Phys., 178, 387

Chan, W. F., Cooper, G., \& Brion, C. E. 1993b, Chem. Phys., 170, 99

Chan, W. F., Cooper, G., Sodhi, R. N. S., \& Brion, C. E. 1993c, Chem. Phys., 170,81

Chapillon, E., Guilloteau, S., Dutrey, A., et al. 2012a, A\&A, 537, A60

Chapillon, E., Dutrey, A., Guilloteau, S., et al. 2012b ApJ, 756, 58

Chen, B.-M., Lu, H.-C., Chen, H.-K., et al. 2006a, ApJ, 647, 1535

Chen, S., Zhou, W., \& Zhang, J. 2006b, Chem. Phys. Lett., 418, 328

Chenel, A., Roncero, O., Aguado, A., et al. 2016, J. Chem. Phys., 144, 144306

Cleeves, L. I., Bergin, E. A., Qi, C., et al. 2015, ApJ, 799, 204

Collings, M. P., Anderson, M. A., Chen, R., et al. 2004, MNRAS, 354, 1133

Cook, P. A., Langford, S. R., Dixon, R. N., \& Ashfold, M. N. R. 2001, J. Chem. Phys., 114, 1672

Cooper, G., Burton, G. R., \& Brion, C. E. 1995, J. Electron Spectrosc. Relat. Phenom., 73, 139

Cooper, G., Anderson, J. E., \& Brion, C. E. 1996, Chem. Phys., 209, 61

Crovisier, J. 1989, A\&A, 213, 459

Cruz-Diaz, G. A., Martín-Doménech, R., Muñoz Caro, G. M., \& Chen, Y.-J. 2016, A\&A, 592, A68

Cunto, W., Mendoza, C., Ochsenbein, F., \& Zeippen, C. J. 1993, A\&A, 275, L5

Cuppen, H. M., Walsh, C., Lamberts, T., et al. 2017, Space Sci. Rev., 212, 1

Daviel, S., Iida, Y., Carnovale, F., \& Brion, C. E. 1984, Chem. Phys., 83, 319

Dehmer, P. M. 1984, Chem. Phys. Lett., 110, 79

Dominik, C., Ceccarelli, C., Hollenbach, D., \& Kaufman, M. 2005, ApJ, 635, L85

Doppmann, G. W., Najita, J. R., Carr, J. S., \& Graham, J. R. 2011, ApJ, 738, 112

Doronin, M., Bertin, M., Michaut, X., et al. 2015, J. Chem. Phys., 143, 084703

Draine, B. T. 1978, ApJS, 36, 595

Draine, B. T., \& Lee, H. M. 1984, ApJ, 285, 89

Driscoll, J. N., \& Warneck, P. 1968, J. Phys. Chem., 72, 3736

Du, F., \& Bergin, E. A. 2014, ApJ, 792, 2

Du, F., Bergin, E. A., Hogerheijde, M., et al. 2017, ApJ, 842, 98

Duflot, D., Robbe, J.-M., \& Flament, J.-P. 1994, J. Chem. Phys., 100, 1236

Dullemond, C. P., \& Dominik, C. 2004, A\&A, 417, 159

Dutrey, A., Guilloteau, S. \& Guélin, M. 1997, A\&A, 317, L55

Dutrey, A., Henning, T., Guilloteau, S., et al. 2007, A\&A, 464, 615

Dutrey, A., Wakelam, V., Boehler, Y., et al. 2011, A\&A, 535, A104

Dutrey, A., Semenov, D., Chapillon, E., et al. 2014, in Protostars and Planets VI, eds. H. Beuther, R. S. Klessen, C. P. Dullemond, \& T. Henning (Arizona: University Arizona Press), 317

Edvardsson, D., Battzer, P., Karlsson, L., et al. 1999, J. Phys. B: At. Mol. Opt. Phys., 32, 2583
Fayolle, E. C., Bertin, M., Romanzin, C., et al. 2011, ApJ, 739, L36 Fayolle, E. C., Bertin, M., Romanzin, C., et al. 2013, A\&A, 556, A122 Fayolle, E. C., Balfe, J., Loomis, R., et al. 2016, ApJ, 816, L28 Fedele, D., Pascucci, I., Brittain, S., et al. 2011, ApJ, 732, 106 Fedele, D., Bruderer, S., van Dishoeck, E. F., et al. 2012, A\&A, 544, L9 Fedele, D., Bruderer, S., van Dishoeck, E. F., et al. 2013, A\&A, 559, A77 Feng, R., Cooper, G., \& Brion, C. E. 1999a, Chem. Phys., 127, 142

Feng, R., Cooper, G., \& Brion, C. E. 1999b, Chem. Phys., 249, 223 Feng, R., Cooper, G., Burton, G. R., et al. 1999c, Chem. Phys., 240, 371

Fillion, J.-H., Dulieu, F., Baouche, S., et al. 2003, J. Phys. B: At. Mol. Opt. Phys., 36,2767

Fillion, J.-H., Ruiz, J., Yang, X.-F., et al. 2004, J. Chem. Phys., 120, 6531

Fillion, J.-H., Fayolle, E. C., Michaut, X., et al. 2014, Faraday Discuss., 168, 533 Fitzpatrick, E. L., \& Massa, D. 2007, ApJ, 663, 320

Florescu-Mitchell, A. I., \& Mitchell, J. B. A. 2006, Phys. Rep., 430, 277

Fogel, J. K. J., Bethell, T. J., Bergin, E. A., et al. 2011, ApJ, 726, 29

France, K., Schindhelm, E., Herczeg, G. J., et al. 2012, ApJ, 756, 17

France, K., Schindhelm, E., Bergin, E. A., et al. 2014, ApJ, 784, 127

Fraser, H. J., Collings, M. P., McCoustra, M. R. S., \& Williams, D. A. 2001, MNRAS, 327, 1165

Fuente, A., Cernicharo, J., Agúndez, M., et al. 2010, A\&A, 524, A19

Fuente, A., Cernicharo, J., \& Agúndez, M. 2012, ApJ, 754, L6

Furuya, K., Nomura, Y., Hersant, F., \& Wakelam, V. 2013, ApJ, 779, 11

Gans, B., Vieira Mendes, L. A., Boyé-Péronne, S., et al. 2010, J. Phys. Chem. A, 114,3237

Gans, B., Boyé-Péronne, S., Broquier, M., et al. 2011, Phys. Chem. Chem. Phys., 13,8140

Garrod, R. T., \& Herbst, E. 2006, A\&A, 457, 927

Geppert, W. D., \& Larsson, M. 2008, Mol. Phys., 106, 16

Gibb, E. L., \& Horne, D. 2013, ApJ, 776, L28

Gibb, E. L., van Brunt, K. A., Brittain, S. D., \& Rettig, T. W. 2007, ApJ, 660, 1572

Gibson, S. T., Greene, J. P., \& Berkowitz, J. 1985, J. Chem. Phys., 83, 4319

Glassgold, A. E., Najita, J. R., \& Igea, J. 2004, ApJ, 615, 972

Glassgold, A. E., Meijerink, R., \& Najita, J. R. 2009, ApJ, 701, 142

Goicoechea, J. R., \& Le Bourlot, J. 2007, A\&A, 467, 1

González García, M., Le Bourlot, J., Le Petit, F., \& Roueff, E. 2008, A\&A, 485, 127

Goumans, T. P. M., Richard, C., Catlow, A., \& Brown, W. A. 2009, MNRAS, 393,1403

Graedel, T. E., Langer, W. D., \& Frerking, M. A. 1982, ApJS, 48, 321

Graninger, D., Öberg, K. I., Qi, C., \& Kastner, J. 2015, ApJ, 807, L15

Gredel, R., Lepp, S., Dalgarno, A., \& Herbst, E. 1989, ApJ, 347, 289

Groth, W. E., Schurath, U., \& Schindler, R. N. 1968, J. Phys. Chem., 72, 3914

Guest, J. A., \& Lee, L. C. 1981, J. Phys. B: At. Mol. Opt. Phys., 14, 3401

Guilloteau, S., Piétu, V., Dutrey, A., \& Guélin, M. 2006, A\&A, 448, L5

Guilloteau, S., Dutrey, A., Wakelam, V., et al. 2012, A\&A, 548, A70

Guilloteau, S., Di Folco, E., Dutrey, A., et al. 2013, A\&A, 549, A92

Guilloteau, S., Reboussin, L., Dutrey, A., et al. 2016, A\&A, 592, A124

Habing, H. J. 1968, Bull. Astron. Inst. Netherlands, 19, 421

Harada, N., Herbst, E., \& Wakelam, V. 2010, ApJ, 721, 1570

Hartquist, T. W., \& Williams, D. A. 1990, MNRAS, 247, 343

Hasegawa, T. I., \& Herbst, E. 1993, MNRAS, 261, 83

Hasegawa, T. I., Herbst, E., \& Leung, C. M. 1992, ApJS, 82, 167

Heays, A. N., Bosman, A. D., \& van Dishoeck, E. F. 2017, A\&A, 602, A105

Heinzeller, D., Nomura, H., Walsh, C., \& Millar, T. J. 2011, ApJ, 731, 115

Henning, Th., \& Semenov, D. 2013, Chem. Rev., 113, 9016

Henning, Th., Semenov, D., Guilloteau, S., et al. 2010, ApJ, 714, 1511

Herczeg, G. J., Valenti, J. A., Johns-Frull, C. M., \& Wood, B. E. 2002, ApJ, 572 , 310

Herzberg, G. 1961, Proc. Roy. Soc. London Ser. A, 262, 291

Hily-Blant, P., Magalhaes, V., Kastner, J., et al. 2017, A\&A, 603, L6

Hogerheijde, M. R., Bergin, E. A., Brinch, C., et al. 2011, Science, 334, 338

Holland, D. M. P., Shaw, D. A., McSweeney, S. M., et al. 1993, Chem. Phys., 173,315

Holland, D. M. P., Shaw, D. A., \& Hayes, M. A. 1995, Chem. Phys., 201, 299

Hollenbach, D., Kaufman, M. J., Bergin, E. A., \& Melnick, G. J. 2009, ApJ, 690, 1497

Huang, J., \& Öberg, K. I. 2015, ApJ, 809, L26

Huang, J., Öberg, K. I., Qi, C., et al. 2017, ApJ, 835, 231

Hudson, R. D. 1971, Rev. Geophys. Space Phys., 9, 305

Huebner, W. F., Keady, J. J., \& Lyon, S. P. 1992, Ap\&SS, 195, 1

Huestis, D. L., \& Berkowitz, J. 2010, BAAS, 42, 972

Hughes, K. J., Turányi, T., Clague, A. R., \& Pilling, M. J. 2001, Int. J. Chem. Kinet., 33, 513

Ilgner, M., \& Nelson, R. P. 2006a, A\&A, 445, 731

Ilgner, M., \& Nelson, R. P. 2006b, A\&A, 445, 223

Ilgner, M., \& Nelson, R. P. 2006c, A\&A, 445, 205 
Ilgner, M., Henning, Th., Markwick, A. J., \& Millar, T. J. 2004, A\&A, 415, 643 Ingleby, L., Calvet, N., Bergin, E., et al. 2009, ApJ, 703, L137

Janssen, L. M. C., van der Loo, M. P. J., Groenenboom, G. C., et al. 2007, J. Chem. Phys., 126, 94304

Jonkheid, B., Dullemond, C. P., Hogerheijde, M. R., \& van Dishoeck, E. F. 2007, A\&A, 463, 203

Jura, M. 1975, ApJ, 197, 575

Kamp, I., \& Dullemond, C. P. 2004, ApJ, 615, 991

Kamp, I., Thi, W.-F., Woitke, P., et al. 2017, A\&A, 607, A41

Kassner, Ch., \& Stuhl, F. 1994, Chem. Phys. Lett., 222, 425

Kastner, J. H., Zuckerman, B., Weintraub, D. A., \& Forveille, T. 1997, Science, 277,67

Kastner, J. H., Hily-Blant, P., Rodriguez, D. R., et al. 2014, ApJ, 793, 55

Kenyon, S. J., \& Hartmann, L. 1995, ApJS, 101, 117

Khamaganov, V., Karunanandan, R., Rodriguez, A., \& Crowley, J. N. 2007, Phys. Chem. Chem. Phys., 9, 4098

Kirby, K., \& Goldfield, E. M. 1991, J. Chem. Phys., 94, 1271

Kirby, K., Roberge, W. G., Saxon, R. P., \& Liu, B. 1980, ApJ, 239, 855

Koch, A. 1997, J. Phys. Chem. A, 101, 1460

Konnov, A. A. 2000, Detailed reaction mechanism for small hydrocarbon combustion, Release 0.5

Kroes, G.-J., van Hemert, M. C., Billing, G. D., \& Neuhauser, D. 1997, J. Chem. Phys., 107, 5757

Kruger, A. J., Richter, M. J., Carr, J. S., et al. 2011, ApJ, 729, 145

Lahuis, F., van Dishoeck, E. F., Boogert, A. C. A., 2016 et al., ApJ, 636, L145

Laor, A., \& Draine, B. T. 1993, ApJ, 402, 441

Lavendy, H., Robbe, J. M., \& Gandara, G. 1987, J. Phys. B: At. Mol. Opt. Phys., 20,3067

Le Bourlot, J., Pineau des Forêts, G., Roueff, E., \& Flower, D. R. 1995, A\&A, 302,870

Le Bourlot, J., Le Petit, F., Pinto, C., et al. 2012, A\&A, 541, A76

Le Petit, F. L., Nehmé, C., Le Bourlot, J., \& Roueff, E. 2006, ApJS, 164, 506

Lee, H.-H., Roueff, E., Pineau des Forêts, G., et al. 1998, A\&A, 334, 1047

Léger, A., Jura, M., \& Omont, A. 1985, A\&A, 144, 147

Li, Y.-J., Zhang, P.-Y., \& Han, K.-L. 2010, J. Mol. At. Sci., 1, 18

Lide, R. D. 2009, CRC Handbook of Chemistry and Physics, 90th edn. (Boca Raton, FL: CRC Press)

Lilly, R. L., Rebbert, R. E., \& Ausloos, P. 1973, J. Photochem., 2, 49

Litorja, M., \& Ruscic, B. 1998, J. Chem. Phys., 108, 6748

Loison, J.-C. 2010, J. Phys. Chem. A, 114, 6515

Loomis, R. A., Cleeves, L. I., Öberg, K. I., et al. 2015, ApJ, 809, L25

Luna, R., Satorre, M. Á., Santonja, C., \& Domingo, M. 2014, A\&A, 566, A27

Macpherson, M. T., Pilling, M. J., \& Smith, M. J. C. 1985, J. Phys. Chem., 89, 2268

Maergoiz, A. I., Nikitin, E. E., \& Troe, J. 2009, Int. J. Mass Spectrom., 280, 42

Markwick, A. J., Ilgner, M., Millar, T. J., \& Henning, Th. 2002, A\&A, 385, 632

Martín-Doménech, R., Muñoz Caro, G. M., Bueno, J., \& Goesmann, F. 2014, A\&A, 564, A8

Martín-Doménech, R., Manzano-Santamaría, J., Muñoz Caro, et al. 2015, A\&A, 584, A14

Martin-Zaïdi, C., Deleuil, M., Le Bourlot, J., et al. 2008, A\&A, 484, 225

Mamajek, E. E. 2005, ApJ, 634, 1385

Manatt, S. L., \& Lane, A. L. 1993, J. Quant. Spectr. Rad. Transf., 50, 267

Mandell, A. M., Mumma, M. J., Blake, G. A., et al. 2008, ApJ, 681, L25

Mandell, A. M., Bast, J., van Dishoeck, E. F., et al. 2016, ApJ, 747, 92

Manion, J. A., Huie, R. E., Levin, R. D., et al., 2013, NIST Chemical Kinetics Database, Data version 2013.03

Mathews, G. S., Klaassen, P. D., Juhász, A., et al. 2013, A\&A, 557, A132

Mathis, J. S., Rumpl, W., \& Nordsieck, K. H. 1977, ApJ, 217, 425

Mathis, J. S., Mezger, P. G., \& Panagia, N. 1983, A\&A, 128, 212

McBride, B. J., Zehe, M. J., \& Gordon, S. 2002, NASA Report TP-2002-211556

McElroy, D., Walsh, C., Markwick, A. J., et al. 2013, A\&A, 550, A36

McMillan, E. C., Shen, G., McCann, J. F., et al. 2016, J. Phys. B: At. Mol. Opt Phys., 49, 084001

McNesby, J. R., Tanaka, I., \& Okabe, H. 1962, J. Chem. Phys., 36, 605

Mebel, A. M., Hayashi, M., Jackson, W. M., et al. 2001, J. Chem. Phys., 114, 9821

Meeus, G., Montesinos, B., Mendigutía, I., et al. 2012, A\&A, 544, A78

Mentall, J. E., Gentieu, E. P., Krauss, M., \& Neumann, D. 1971, J. Chem. Phys. 55,5471

Montesinos, B., Eiroa, C., Mora, A., \& Merín, B. 2009, A\&A, 495, 901

Mota, R., Parafita, R., Giuliani, A., et al. 2005, Chem. Phys. Lett., 416, 152

Muñoz Caro, G. M., Jiménez-Escobar, A., Martín-Gago, J. Á., et al. 2010, A\&A, 522, A 108

Najita, J. R., Carr, J. S., Strom, S. E., et al. 2010, ApJ, 712, 274

Najita, J. R., Carr, J. S., Pontoppidan, K. M., et al. 2013, ApJ, 766, 134

Nee, J. B., \& Lee, J. C. 1984, J. Chem. Phys., 81, 31

Nee, J. B., \& Lee, L. C. 1986, J. Chem. Phys., 84, 5303
Nee, J. B., Suto, M., \& Lee, L. C. 1985, J. Phys. B: At. Mol. Opt. Phys., 18, L293 Neufeld, D. A., \& Wolfire, M. G. 2009, ApJ, 706, 1594

Noble, J. A., Congiu, E., Dulieu, F., \& Fraser, H. J. 2012a, MNRAS, 421, 768

Noble, J. A., Theule, P., Mispelaer, F., et al. 2012b, A\&A, 543, A5

North, S. W., Blank, D. A., Chu, P. M., \& Lee, Y. T. 1995, J. Chem. Phys., 102, 792

Norwood, K., \& Ng, C. Y. 1989, Chem. Phys. Lett., 156, 145

Norwood, K., Nourbakhsh, S., He, G.-H., \& Ng, C. Y. 1991, Chem. Phys. Lett., 184,147

Nuth, J. A., \& Glicker, S. 1982, J. Quant. Spectr. Rad. Transf., 28, 223

Öberg, K. I., Linnartz, H., Visser, R., \& van Dishoeck, E. F. 2009, ApJ, 693 1209

Öberg, K. I., Qi, C., Fogel, J. K. J., et al. 2010, ApJ, 720, 480

Öberg, K. I., Qi, C., Fogel, J. K. J., et al. 2011, ApJ, 734, 98

Öberg, K. I., Guzmán, V. V., Furuya, K., et al. 2015, Nature, 520, 198

Öberg, K. I., Guzmán, V. V., Merchant, C. J., et al. 2017, ApJ, 839, 43

Okabe, H., \& Lenzi, M. 1967, J. Chem. Phys., 47, 5241

Pacheco-Vázquez, S., Fuente, A., Agúndez, M., et al. 2015, A\&A, 578, A81

Pacheco-Vázquez, S., Fuente, A., Baruteau, C., et al. 2016, A\&A, 589, A60

Pascucci, I., Herczeg, G., Carr, J. S., \& Bruderer, S. 2013, ApJ, 779, 178

Phillips, L. F. 1981, J. Phys. Chem., 85, 3994

Piétu, V., Dutrey, A., \& Guilloteau, S. 2007, A\&A, 467, 163

Podio, L., Kamp, I., Codella, C., et al. 2013, ApJ, 766, L5

Pontoppidan, K. M., Salyk, C., Blake, G. A., et al. 2010a, ApJ, 720, 887

Pontoppidan, K. M., Salyk, C., Blake, G. A., \& Käufl, H. U. 2010b, ApJ, 722, L173

Pontoppidan, K. M., Salyk, C., Bergin, E. A., et al. 2014, in Protostars and Planets VI, eds. H. Beuther, R. S. Klessen, C. P. Dullemond, \& T. Henning (Arizona: University Arizona Press), 363

Pouilly, B., Robbe, J. M., Schamps, J., \& Roueff, E. 1983, J. Phys. B: At. Mol. Opt. Phys., 16, 437

Prasad, S. S., \& Tarafdar, S. P. 1983, ApJ, 267, 603

Qi, C., Kessler, J. E., Koerner, D. W., et al. 2003, ApJ, 597, 986

Qi, C., Wilner, D. J., Aikawa, Y., et al. 2008, ApJ, 681, 1396

Qi, C., Öberg, K. I., \& Wilner, D. J. 2013a, ApJ, 765, 34

Qi, C., Öberg, K. I., Wilner, D. J., \& Rosenfeld, K. A. 2013b, ApJ, 765, L14

Qi, C., Öberg, K. I., Wilner, D. J., et al. 2013c, Science, 341, 630

Qi, C., Öberg, K. I., Andrews, S. M., et al. 2015, ApJ, 813, 128

Riviere-Marichalar, P., Ménard, F., Thi, W.-F., et al. 2012, A\&A, 538, L3

Roberge, W. G., Jones, D., Lepp, S., \& Dalgarno, A. 1991, ApJS, 77, 287

Roberge, A., Lecavelier des Etangs, A., Grady, C. A., et al. 2001, ApJ, 551, L97

Roueff, E., Alekseyev, A. B., \& Le Bourlot, J. 2014, A\&A, 566, A30

Ruscic, B., \& Berkowitz, J. 1983, Phys. Rev. Lett., 50, 675

Salinas, V. N., Hogerheijde, M. R., Bergin, E. A., et al. 2016, A\&A, 591, A122

Salyk, C., Blake, G. A., Boogert, A. C. A., \& Brown, J. M. 2007, ApJ, 655, L105

Salyk, C., Pontoppidan, K. M., Blake, G. A., et al. 2008, ApJ, 676, L49

Salyk, C., Pontoppidan, K. M., Blake, G. A., et al. 2011, ApJ, 731, 130

Sander, S. P. J., Abbatt, J. R., Barker, J. B., et al. 2011, JPL Publication 10-6

Sandford, S. A., \& Allamandola, L. J. 1993, ApJ, 417, 815

Sargent, B. A., Forrest, W., Watson, D. M., et al. 2014 ApJ, 792, 83

Sarrasin, E., Abdalah, D. B., Wernli, M., et al. 2010, MNRAS, 404, 518

Schreyer, K., Guilloteau, S., Semenov, D., et al. 2008, A\&A, 491, 821

Schriver-Mazzuoli, L., Chaabouni, H., \& Schriver, A. 2003, J. Mol. Struct., 644, 151

Schwarz, K. R., Bergin, E. A., Cleeves, L. I., et al. 2016, ApJ, 823, 91

Semenov, D., \& Wiebe, D. 2011, ApJS, 196, 25

Semenov, D., Wiebe, D., \& Henning, Th. 2004, A\&A, 417, 93

Semenov, D., Wiebe, D., \& Henning, Th. 2006, ApJ, 647, L57

Shen, C. J., Greenberg, J. M., Schutte, W. A., \& van Dishoeck, E. F. 2004, A\&A, 415,203

Slanger, T. G., \& Black, G. 1982, J. Chem. Phys., 77, 2432

Smith, I. W. M., Sage, A. M., Donahue, N. M., et al. 2006, Faraday Discuss., 133,137

Sorkhabi, O., Blunt, V. M., Lin, H., et al. 1997, J. Chem. Phys., 107, 9842

Stephens, J. A., \& McKoy, V. 1988, J. Chem. Phys., 88, 1737

Stark, G., Yoshino, K., \& Smith, P. L. 1987, J. Mol. Spectr., 124, 420

Stief, L. J., Donn, B., Glicker, S., et al. 1972, ApJ, 171, 21

Su, T., \& Chesnavich, W. J. 1982, J. Chem. Phys., 76, 5183

Teague, R., Semenov, D., Guilloteau, S., et al. 2015, A\&A, 574, A137

Taatjes, C. A., Osborn, D. L., Selby, T. M., et al. 2008, J. Phys. Chem. A, 112, 9336

Telleschi, A., Güdel, M., Briggs, K. R., et al. 2007, A\&A, 468, 541

Thi, W.-F., van Zadelhoff, G.-J., \& van Dishoeck, E. F. 2004, A\&A, 425, 955

Thi, W.-F., Woitke, P., \& Kamp, I. 2010, MNRAS, 407, 232

Thi, W.-F., Ménard, F., Meeus, G., et al. 2011, A\&A, 530, L2

Toffoli, D., \& Lucchese, R. R. 2004, J. Chem. Phys., 120, 6010

van Dishoeck, E. F. 1987, J. Chem. Phys., 86, 196

van Dishoeck, E. F., \& Dalgarno, A. 1984, ApJ, 277, 576 
van Dishoeck, E. F., Beärda, R. A., \& van Hemert, M. C. 1996, A\&A, 307, 645 van Dishoeck, E. F., Thi, W.-F., \& van Zadelhoff, G.-J. 2003, A\&A, 400, L1 van Dishoeck, E. F., Jonkheid, B., \& van Hemert, M. C. 2006, Faraday Discuss., 133,231

van Dishoeck, E. F., Bergin, E. A., Lis, D. C., \& Lunine, J. I. 2014, in Protostars and Planets VI, eds. H. Beuther, R. S. Klessen, C. P. Dullemond, \& T. Henning (Arizona: University Arizona Press), 835

van Hemert, M. C., \& van Dishoeck, E. F. 2008, Chem. Phys., 343, 292

van Zadelhoff, G.-J., van Dishoeck, E. F., Thi, W.-F., \& Blake, G. A. 2001, A\&A, 377,566

van Zadelhoff, G.-J., Aikawa, Y., Hogerheijde, M. R., \& van Dishoeck, E. F. 2003, A\&A, 397, 789

Vasyunin, A. I., Semenov, D., Henning, Th., et al. 2008, ApJ, 672

Venot, O., Fray, N., Bénilan, Y., et al. 2013, A\&A, 551, A131

Verner, D. A., Ferland, G. J., Korista, K. T., \& Yakovlev, D. G. 1996, ApJ, 465 487

Vetter, R., Zülicke, L., Koch, A., et al. 1996, J. Chem. Phys., 104, 5558

Wakelam, V., Smith, I. W. M., Herbst, E., et al. 2010, Space Sci. Rev., 156, 13

Wakelam, V., Herbst, E., Loison, J.-C., et al. 2012, ApJS, 199, 21

Wakelam, V., Loison, J.-C., Herbst, E., et al. 2015, ApJS, 217, 20

Walsh, C., Millar, T. J., \& Nomura, H. 2010, ApJ, 722, 1607

Walsh, C., Nomura, H., Millar, T. J., \& Aikawa, Y. 2012, ApJ, 747, 114

Walsh, C., Millar, T. J., Nomura, H., et al. 2014, A\&A, 563, A33

Walsh, C., Nomura, H., \& van Dishoeck, E. 2015, A\&A, 582, A88

Walsh, C., Loomis, R. A., Öberg, K. I., et al. 2016, ApJ, 823, L10

Wang, K., Stephens, J. A., \& McKoy, V. 1990, J. Chem. Phys., 93, 7874
Wang, J., Yang, B., Cool, T. A., et al. 2008, Int. J. Mass Spectrom., 269, 210 Watanabe, K., Matsunaga, F. M., \& Sakai, H. 1967, Appl. Opt., 6, 391

Webb, R. A., Zuckerman, B., Platais, I., et al. 1999, ApJ, 512, L63

Weingartner, J. C., \& Draine, B. T. 2001, ApJ, 548, 296

Wheeler, M. D., Orr-Ewing, A. J., \& Ashfold, M. N. R. 1997, J. Chem. Phys., 107,7591

Willacy, K. 2007, ApJ, 660, 441

Willacy, K., \& Langer, W. D. 2000, ApJ, 544, 903

Willacy, K., \& Woods, P. M. 2009, ApJ, 703, 479

Willacy, K., Khlar, H. H., Millar, T. J., \& Henning, Th. 1998, A\&A, 338, 995

Willacy, K., Langer, W., Allen, M., \& Bryden, G. 2006, ApJ, 644, 1202

Wilson, S. H. S., Howe, J. D., Rosser, K. N., et al. 1994, Chem. Phys. Lett., 227, 456

Woitke, P., Kamp, I., \& Thi, W.-F. 2009, A\&A, 501, 383

Woitke, P., Min, M., Pinte, C., et al. 2016, A\&A, 586, A103

Woodall, J., Agúndez, M., Markwick-Kemper, A. J., \& Millar, T. J. 2007, A\&A 466, 1197

Woods, P. M., \& Willacy, K. 2007, ApJ, 655, L49

Wu, C. Y. R., Yang, B. W., Chen, F. Z., et al. 2000, Icarus, 145, 289

Wu, G., Jiang, B., Ran, Q., et al. 2004, J. Chem. Phys., 120, 2193

Wu, Y.-J., Lu, H.-C., Chen, H.-K., et al. 2007, J. Chem. Phys., 127, 154311

Yang, L., Ciesla, F. J., \& Alexander, C. M. O’D. 2013, Icarus, 226, 256

Yoshino, K., Parkinson, W. H., Ito, K., \& Matsui, T. 2005, J. Mol. Spectr., 229, 238

Zanchet, A., Agúndez, M., Herrero, V. J., et al. 2013, AJ, 146, 125

Zhang, K., Bergin, E. A., Blake, G. A., et al. 2017, Nat. Astron., 1, 0130 


\section{Appendix A: Photodissociation and photoionization cross sections}

Photodissociation and photoionization processes are treated using the relevant cross section as a function of wavelength. We adopt this approach for key species for which cross section data are available in the literature, excluding $\mathrm{H}_{2}$ and $\mathrm{CO}$, the photodissociation of which are treated solving the excitation and line-by-line radiative transfer and taking into account self-shielding effects. We are mainly interested in cross sections ranging from the Lyman cutoff, at $911.776 \AA$, to wavelengths in the range 1500-4000 $\AA$, where most molecules have their photodissociation threshold. We note that in protoplanetary disks, stellar photons with wavelengths shorter than the Lyman cutoff may also have an impact on the photodissociation and photoionization rates in disk regions exposed to the star. It will be worth taking this into account in the future.

Cross sections of molecules with a closed electronic shell have been mostly taken from experimental studies carried out at room temperature. In the case of radicals, most of the cross section data come from theoretical studies. We note that cross sections can show a great dependence with temperature, something that may have an important impact on the chemistry of protoplanetary disks taking into account that the gas kinetic temperature can take values from hundreds to thousands of degrees Kelvin in the photon-dominated region of the disk. Of course it would be desirable to use temperature-dependent cross sections, although such data are currently limited to just a few species (e.g., Venot et al. 2013; McMillan et al. 2016). This will be something to take into account in the future.

We have compiled photo cross sections for 29 molecules and 8 atoms from original sources in the literature or from databases such as the MPI-Mainz UV/VIS Spectral Atlas of Gaseous Molecules of Atmospheric Interest ${ }^{14}$, which contains extensive information on experimental cross sections of stable molecules, the Photo Rate Coefficient Database ${ }^{15}$ (Huebner et al. 1992), the Leiden database of photodissociation and photoionization of astrophysically relevant molecules ${ }^{16}$ (van Dishoeck et al. 2006; Heays et al. 2017), which contains theoretical data of numerous radicals. It is common that experimental studies provide the photoabsorption cross section without disentangling whether the absorption leads to fluorescence, dissociation, or ionization. Unless otherwise stated, we have assumed that absorption of FUV photons not leading to ionization leads to dissociation of the molecule, that is, that the contribution of fluorescence to the total photoabsortion cross section is negligible. This may be a bad approximation at long wavelengths but is likely to be correct at short wavelengths, typically below $1500 \AA$. In the case of photoionization of atoms we have made use of databases such as TOPbase, the Opacity Project atomic database ${ }^{17}$ (Cunto et al. 1993), to retrieve cross sections for some of the atoms. In the following subsections we detail the cross section adopted for each of the 29 molecules and 8 atoms considered. In Fig. A.1 we show the photodissociation cross section as a function of wavelength for some key molecules.

\section{A.1. $\mathrm{CH}$}

The photodissociation cross section of methylidyne has been taken from the calculations of van Dishoeck (1987), which

\footnotetext{
${ }^{14}$ http://wwW.uv-vis-spectral-atlas-mainz.org

15 http://phidrates. space.swri.edu/

16 http://home.strw. leidenuniv.nl/\$ sim\$ewine/photo/

17 http://cdsweb.u-strasbg.fr/topbase/topbase.html
}

comprise dissociation by lines and by continuum. The photoionization cross section has been obtained from the theoretical study by Barsuhn \& Nesbet (1978). The photoionization threshold of $\mathrm{CH}$ is $1170 \AA$ (Huebner et al. 1992).

\section{A.2. $\mathrm{CH}^{+}$}

The photodissociation cross section of $\mathrm{CH}^{+}$is taken from the theoretical calculations of Kirby et al. (1980), where we have adopted a cross section of $10 \mathrm{Mb}^{18}$ at the resonances lying at 1523, 1546, and $1579 \AA$. Photodissociation through the three excited electronic states considered by Kirby et al. (1980), $2^{1} \Sigma^{+}$, $2^{1} \Pi$, and $3^{1} \Sigma^{+}$, yields $\mathrm{C}+\mathrm{H}^{+}$as products.

\section{A.3. $\mathrm{CH}_{2}$}

The photodissociation cross section of the methylene radical has been taken from van Dishoeck et al. (1996), whose calculations comprise dissociation by lines and by continuum radiation. Methylene has two main dissociation channels:

$$
\begin{aligned}
\mathrm{CH}_{2}+h v & \rightarrow \mathrm{CH}+\mathrm{H} \\
& \rightarrow \mathrm{C}+\mathrm{H}_{2},
\end{aligned}
$$

but the latter is a minor one (Kroes et al. 1997), and has therefore been neglected here. The ionization threshold of $\mathrm{CH}_{2}$ is $1193 \AA$, although there are no cross section data in the literature, except for the relative data in the ionization threshold region by Litorja \& Ruscic (1998).

\section{A.4. $\mathrm{CH}_{3}$}

The UV spectra of $\mathrm{CH}_{3}$ has been recorded in photographic plates (Herzberg 1961; Callear \& Metcalfe 1976), although no quantitative measurement of the intensity was carried out. More recently, the absolute photodissociation cross section was measured in the 2000-2400 $\AA$ region by Cameron et al. (2002) and in the band at $2164 \AA$ by Khamaganov et al. (2007), in good agreement with previous absolute measurements: $40.1-41.2 \mathrm{Mb}$ at $2136.6 \AA$ (Macpherson et al. 1985) and 35.1 Mb at $2164 \AA$ (Arthur 1986). We have adopted the photodissociation cross section measured by Cameron et al. (2002) and Khamaganov et al. (2007) at wavelengths longer than $2000 \AA$, and have calibrated the band intensities classified in five categories, from very weak to very strong, by Herzberg (1961) based on the absolute cross section measured for the $2164 \AA$ band. The dissociation of the methyl radical has two possible channels:

$$
\begin{aligned}
\mathrm{CH}_{3}+h v & \rightarrow \mathrm{CH}_{2}+\mathrm{H} \\
& \rightarrow \mathrm{CH}+\mathrm{H}_{2},
\end{aligned}
$$

whose quantum yields are not clear. Channel (A.3) is observed at $2163 \AA$ by Wilson et al. (1994), at $1933 \AA$ by North et al. (1995), and at $2125 \AA$ by Wu et al. (2004), while channel (A.4) is observed through fluorescence of $\mathrm{CH}$ at $\lambda>1050 \AA$ by Kassner \& Stuhl (1994). In the absence of better constraints we have assumed a quantum yield of $50 \%$ for each channel. As concerns photoionization, we adopt the absolute cross section measured by Gans et al. (2010) from the photoionization threshold $(1260 \AA$ ) to $1085 \AA$, and consider a constant cross section shortward of $1085 \AA$. The adopted cross section is, within uncertainties, similar to those reported in previous studies (Taatjes et al. 2008; Loison 2010).

$\overline{18} 1 \mathrm{Mb}$ is equal to $10^{-18} \mathrm{~cm}^{2}$. 

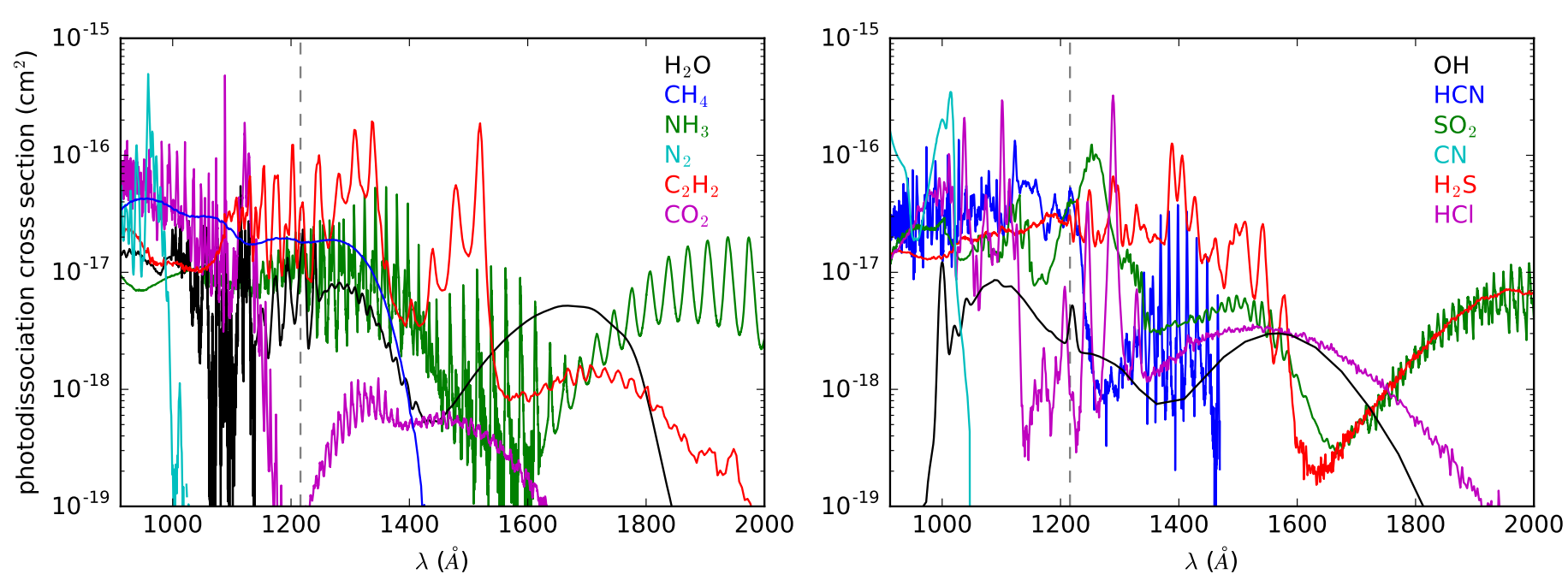

Fig. A.1. Photodissociation cross section of various molecules as a function of wavelength. The position of the Ly- $\alpha$ line at $1216 \AA$ is marked with a vertical dashed line.

\section{A.5. $\mathrm{CH}_{4}$}

In the case of methane the photoabsorption cross section has been taken from the experimental study by Au et al. (1993) while the photoionization cross section (the ionization threshold of $\mathrm{CH}_{4}$ is $983.2 \AA$ ) has been measured by Wang et al. (2008). Methane has various photodissociation channels leading to the radicals $\mathrm{CH}_{3}, \mathrm{CH}_{2}$, and $\mathrm{CH}$ :

$$
\begin{aligned}
\mathrm{CH}_{4}+h v & \rightarrow \mathrm{CH}_{3}+\mathrm{H} \\
& \rightarrow \mathrm{CH}_{2}\left(X^{3} B_{1}\right)+\mathrm{H}+\mathrm{H} \text { or } \mathrm{CH}_{2}\left(a^{1} A_{1}\right)+\mathrm{H}_{2}, \\
& \rightarrow \mathrm{CH}+\mathrm{H}_{2}+\mathrm{H} \text { or } \mathrm{CH}+\mathrm{H}+\mathrm{H}+\mathrm{H},
\end{aligned}
$$

whose quantum yields have been measured by Gans et al. (2011) at 1182 and $1216 \AA$.

\section{A.6. $C_{2}$}

The photodissociation and photoionization cross sections of $\mathrm{C}_{2}$ have been taken from the theoretical studies by Pouilly et al. (1983) and Toffoli \& Lucchese (2004), respectively. The photoionization threshold of $\mathrm{C}_{2}$ is $1020 \AA$ (Toffoli \& Lucchese 2004).

\section{A.7. $\mathrm{C}_{2} \mathrm{H}$}

In the case of the ethynyl radical we adopt the photodissociation cross section by lines calculated by van Hemert \& van Dishoeck (2008). The main dissociation channel yields $\mathrm{C}_{2}+\mathrm{H}$ (Duflot et al. 1994; Sorkhabi et al. 1997; Mebel et al. 2001; Apaydin et al. 2004). The photoionization threshold of $\mathrm{C}_{2} \mathrm{H}$ is $1068 \AA$ (Lide 2009), although to our knowledge there are not cross section data available in the literature.

\section{A.8. $\mathrm{C}_{2} \mathrm{H}_{2}$}

For acetylene the photoionization cross section at wavelengths shorter than the ionization threshold of $1087.6 \AA$ has been taken from the compilation by Hudson (1971). We have subtracted the photoionization cross section from the photoabsorption cross section measured by Cooper et al. (1995) to obtain the photodissociation cross section, which is assumed to yield the $\mathrm{C}_{2} \mathrm{H}$ radical with a $100 \%$ efficiency.

\section{A.9. $\mathrm{OH}$}

The photoabsorption cross section of the hydroxyl radical has been measured by Nee \& Lee (1984) in the wavelength range 1150-1830 A. On the theoretical side, the photodissociation has also been studied by van Dishoeck \& Dalgarno (1984). The experimental values are somewhat higher than the theoretical ones, although calculations indicate that photodissociation by lines is also important at wavelengths shorter than $1150 \AA$. We adopt the theoretical photodissociation cross section, mainly because it spans over a broader wavelength range. Relative photoionization cross section has been measured by Dehmer (1984) up to the threshold at $952.5 \AA$ (Lide 2009). The absolute scale of the photoionization cross section is fixed by setting a value of 2.5 $\mathrm{Mb}$ at $946 \AA$, according to the calculations of Stephens \& McKoy (1988).

\section{A.10. $\mathrm{H}_{2} \mathrm{O}$}

The photoabsorption cross section of water has been taken from various experimental studies: Fillion et al. (2004) for the wavelength range 999-1139 $\AA$, Mota et al. (2005) in the wavelength range 1148-1939 $\AA$, and Chan et al. (1993a) elsewhere in the range from the Lyman cutoff to $2060.5 \AA$. The photoionization cross section for wavelengths shorter than the ionization threshold of $\mathrm{H}_{2} \mathrm{O}(982.4 \AA)$ has been taken from the experimental study of Fillion et al. (2003). Water has two main photodissociation channels, yielding $\mathrm{OH}$ radicals and $\mathrm{O}$ atoms:

$$
\begin{aligned}
\mathrm{H}_{2} \mathrm{O}+h v & \rightarrow \mathrm{OH}+\mathrm{H}, \\
& \rightarrow \mathrm{O}\left({ }^{3} P\right)+\mathrm{H}+\mathrm{H} \text { or } \mathrm{O}\left({ }^{1} D\right)+\mathrm{H}_{2},
\end{aligned}
$$

where channel (A.8) dominates over channel (A.9). Water can be photodissociated in the first band $\tilde{X}^{1} A_{1}-\tilde{A}^{1} B_{1}$ at wavelengths longer than $1360 \AA$ and in the second $\tilde{X}^{1} A_{1}-\tilde{B}^{1} A_{1}$ and higher bands at shorter wavelengths. Quantum yields of $78 \%$ and 22\% for channels (A.8) and (A.9) have been measured by Slanger \& Black (1982) at $1216 \AA$ (Ly $\alpha$ ). We adopt these values for $\lambda<1360 \AA$ while at longer wavelengths we take quantum yields of $99 \%$ and $1 \%$ (Crovisier 1989). 


\section{A.11. $\mathrm{O}_{2}$}

In the wavelength range of photoionization of molecular oxygen (up to the ionization threshold of $1027.8 \AA$ A, e.g., Huebner et al. 1992), the photoabsorption and photoionization cross sections have been taken from the experimental study of Holland et al. (1993). Up to $1750 \AA$, the photoabsorption cross section has been taken from Yoshino et al. (2005), and from Chan et al. (1993b) at longer wavelengths. The dissociation threshold of $\mathrm{O}_{2}$ is $2423.7 \AA$ (Huebner et al. 1992), although at wavelengths longer than $\sim 1850 \AA$ the photodissociation cross section already becomes negligible.

\section{A.12. $\mathrm{H}_{2} \mathrm{CO}$}

The photoabsorption cross section of formaldehyde is taken from measurements by Cooper et al. (1996). The cross section of photoionization, which occurs at wavelengths shorter than $\sim 1400 \AA$, has been measured by Mentall et al. (1971). The photodissociation channels of $\mathrm{H}_{2} \mathrm{CO}$ are

$$
\begin{aligned}
\mathrm{H}_{2} \mathrm{CO}+h v & \rightarrow \mathrm{CO}+\mathrm{H}_{2}, \\
& \rightarrow \mathrm{CO}+\mathrm{H}+\mathrm{H}, \\
& \rightarrow \mathrm{HCO}+\mathrm{H},
\end{aligned}
$$

where channel (A.12) can be neglected as it occurs in the wavelength range 2000-3340 $\AA$, where the absorption cross section of $\mathrm{H}_{2} \mathrm{CO}$ is three orders of magnitude lower than at $\lambda<2000 \AA$. Channels (A.10) and (A.11) occur with similar quantum yields (Stief et al. 1972), however, since the implications of producing either molecular or atomic hydrogen in the photodissociation of $\mathrm{H}_{2} \mathrm{CO}$ are small for the chemistry of protoplanetary disks, we have assumed that only channel (A.10) occurs.

\section{A.13. $\mathrm{CO}_{2}$}

The ionization threshold of $\mathrm{CO}_{2}$ is $900 \AA$ and thus photoionization does not occur in our wavelength range of interest. The adopted photoabsorption cross section is based on the critical evaluation by Huestis \& Berkowitz (2010). The only allowed channel in the photodissociation of carbon dioxide is that yielding CO + O (see, e.g., Huebner et al. 1992).

\section{A.14. NH}

In the case of the $\mathrm{NH}$ radical the photodissociation cross section has been calculated by Kirby \& Goldfield (1991). The photoionization cross section has been calculated by Wang et al. (1990) to be around $8 \mathrm{Mb}$ in the narrow wavelength range from the Lyman cutoff to the ionization threshold at $919.1 \AA$.

\section{A.15. $\mathrm{NH}_{2}$}

The photodissociation cross section of $\mathrm{NH}_{2}$ is taken from the theoretical calculations of Koch (1997). There are two possible dissociation channels:

$$
\begin{aligned}
\mathrm{NH}_{2}+h v & \rightarrow \mathrm{NH}+\mathrm{H}, \\
& \rightarrow \mathrm{N}+\mathrm{H}_{2},
\end{aligned}
$$

where only channel (A.13) is considered here, based on experimental and theoretical evidence that the major channel in the photodissociation of $\mathrm{NH}_{2}$ leads to $\mathrm{NH}$ radicals (Biehl et al.
1994; Vetter et al. 1996). As concerns photoionization, we used the relative cross section measured by Gibson et al. (1985) in the wavelength range 745-1125 $\AA$ (the ionization threshold is $1130 \AA$ ) and fixed the absolute scale by assuming a guess value of $10 \mathrm{Mb}$ for the cross section at $912 \AA$.

\section{A.16. $\mathrm{NH}_{3}$}

The photoabsorption cross section of ammonia is taken from Edvardsson et al. (1999) up to the ionization threshold of $1231 \AA$, from $\mathrm{Wu}$ et al. (2007) in the wavelength range 1231-1440 $\AA$, and from Chen et al. (2006a) at longer wavelengths. The data measured by Burton et al. (1993) at a lower spectral resolution is consistent with the data described above. The photoionization cross section has been taken from Edvardsson et al. (1999). As concerns photodissociation, there are different channels for ammonia, leading to $\mathrm{NH}_{2}$ and $\mathrm{NH}$ radicals,

$$
\begin{aligned}
\mathrm{NH}_{3}+h v & \rightarrow \mathrm{NH}_{2}+\mathrm{H}, \\
& \rightarrow \mathrm{NH}\left(X^{3} \Sigma^{-}\right)+\mathrm{H}+\mathrm{H} \text { or } \mathrm{NH}\left(a^{1} \Delta\right)+\mathrm{H}_{2},
\end{aligned}
$$

where channel (A.15) dominates over the production of $\mathrm{NH}$ with a yield $\geq 0.694$. The quantum yields over the wavelength range of interest have been taken from various measurements carried out at different wavelengths (McNesby et al. 1962; Okabe et al. 1967; Groth et al. 1968; Lilly et al. 1973; Slanger \& Black 1982).

\section{A.17. $\mathrm{N}_{2}$}

Molecular nitrogen is ionized by photons with energies higher than $15.58 \mathrm{eV}$ and thus only photodissociation, and not photoionization, occurs in our wavelength range of interest. The photoabsorption cross section has been taken from the experiments carried out by Chan et al. (1993c). The photodissociation threshold of $\mathrm{N}_{2}$ is $1270.85 \AA$, although in the practice only photons with $\lambda<1000 \AA$ are efficient enough in dissociating molecular nitrogen.

\section{A.18. $\mathrm{CN}$}

The photodissociation cross section of the $\mathrm{CN}$ radical has been calculated by Lavendy et al. (1987). The photodissociation threshold is $1600 \AA$ (Huebner et al. 1992), although in the practice the cross section becomes vanishingly small at wavelengths longer than $\sim 1100 \AA$. Since the ionization threshold of $\mathrm{CN}$ is $911.756 \AA$ (Lide 2009), slightly shorter than that of hydrogen, we do not consider photoionization here.

\section{A.19. HCN}

Hydrogen is ionized by photons with wavelengths shorter than the Lyman cutoff and thus photoionization of HCN is not considered here. We have adopted the photoabsorption cross section measured by Nuth \& Glicker (1982) up to $\lambda=1469.2 \AA$. We assume that at longer wavelengths the contribution to photodissociation is small and that the main photodissociation channel leads to the $\mathrm{CN}$ radical.

\section{A.20. HNC}

The photoabsorption cross section of HNC has recently been calculated simultaneously with that of the most stable isomer $\mathrm{HCN}$ by Chenel et al. (2016) in the 7-10 eV energy range. More recently, Aguado et al. (2017) have extended those calculations 
including higher electronic states to cover the 7-13.6 eV energy range. We adopted the cross section calculated by Aguado et al. (2017) and assumed that absorption in the studied wavelength range leads mainly to dissociation rather than fluorescence. The ionization threshold of HNC is $992 \AA$ (Lide 2009), although the relevant cross section is not known.

\section{A.21. NO}

The photoabsorption cross section of nitric oxide was taken from Watanabe et al. (1967) up to $1350 \AA$ and from Guest \& Lee (1981) at longer wavelengths. The photoionization cross section was measured by Watanabe et al. (1967) up to the ionization threshold, at $1340 \AA$. The photodissociation cross section was obtained by subtracting the cross sections due to photoionization (Watanabe et al. 1967) and to fluorescence (as characterized by Guest \& Lee 1981) from the total photoabsorption cross section.

\section{A.22. SH}

In the case of the mercapto radical, various theoretical studies have investigated the photodissociation dynamics of selected electronic states, mostly the first excited state $A^{2} \Sigma^{+}$but also higher ones such as ${ }^{2} \Sigma^{-},{ }^{2} \Delta$, and $2^{2} \Pi$ (e.g., Wheeler et al. 1997; Chen et al. 2006b; Janssen et al. 2007). However, no quantitative measurement of the photodissociation cross section across the FUV range is available. We have therefore adopted the photodissociation cross section from the Leiden database, whose data are largely based on the calculations of Bruna \& Hirsch (1987). The photoionization threshold of SH is $1190 \AA$, although there are no data on the relevant cross section.

\section{A.23. $\mathrm{SH}^{+}$}

For the $\mathrm{SH}^{+}$ion, the photodissociation cross section was taken from the Leiden database, where the recent calculations by McMillan et al. (2016) were used adopting the cross section of photodissociation from the ground $v=0$ and $J=0$ level (see Heays et al. 2017). Photodissociation at FUV wavelengths is dominated by transitions involving the excited electronic states $3^{3} \Sigma^{-}$and $3^{3} \Pi$, which yield $\mathrm{S}+\mathrm{H}^{+}$as products (McMillan et al. 2016).

\section{A.24. $\mathrm{H}_{2} \mathrm{~S}$}

For hydrogen sulfide the photoabsorption cross section has been taken from Feng et al. (1999a) and the photoionization yield from Feng et al. (1999b). We just consider the dissociation channel leading to $\mathrm{SH}+\mathrm{H}$, which dominates over the others (see, e.g., Cook et al. 2001). There are two possible ionization channels (Huebner et al. 1992):

$$
\begin{aligned}
\mathrm{H}_{2} \mathrm{~S}+h v & \rightarrow \mathrm{H}_{2} \mathrm{~S}^{+}+\mathrm{e}^{-}, \\
& \rightarrow \mathrm{S}^{+}+\mathrm{H}_{2}+\mathrm{e}^{-},
\end{aligned}
$$

where channel (A.17) has a threshold of $1185.25 \AA$ and dominates, while channel (A.18) has a threshold of $927 \AA$ and accounts for just $5 \%$ of the total ionization quantum yield. We therefore consider that ionization yields $\mathrm{H}_{2} \mathrm{~S}^{+}$in all cases.

\section{A.25. CS}

In the case of carbon monosulfide, the photodissociation cross section was taken from the Leiden database, whose data are based on absorption lines measurements by Stark et al. (1987) and vertical excitation energies calculated by Bruna et al. (1975), in addition to some oscillator strength guesses. For the photoionization cross section, we used the relative measurements carried out by Norwood et al. (1991) in the wavelength range $1000-1100 \AA$ (the ionization threshold is $1095.5 \AA$ ) and fixed the absolute scale by assuming a guess value of $10 \mathrm{Mb}$ for the cross section at $\leq 1000 \AA$.

\section{A.26. SO}

For sulfur monoxide, the cross section data available in the literature cover just a limited spectral region. The photoabsorption cross section has been measured in the wavelength ranges

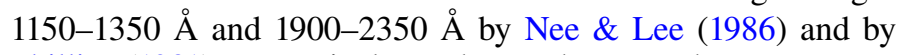
Phillips (1981), respectively. In the gap between these two spectral regions and at wavelengths shorter than $1150 \AA$ we have adopted an arbitrary photodissociation cross section of $5 \mathrm{Mb}$. The ionization threshold of SO is $1205 \AA$ (Huebner et al. 1992). As photoionization cross section we used the photoelectron spectrum measured by Norwood \& Ng (1989) in the wavelength range 1025-1225 $\AA$ and scaled it assuming a cross section of $10 \mathrm{Mb}$ at $\leq 1025 \AA$.

\section{A.27. $\mathrm{SO}_{2}$}

The photoabsorption cross section of sulfur dioxide is based on various experimental studies dealing with different wavelength ranges: Holland et al. (1995) up to the ionization threshold of $\mathrm{SO}_{2}$ at $1004 \AA$, Feng et al. (1999c) in the range 1004-1061 $\AA$, Manatt \& Lane (1993) in the range 1061-1717.7 $\AA$, and Wu et al. (2000) at longer wavelengths. The photoionization cross section is taken from Holland et al. (1995). The photodissociation of $\mathrm{SO}_{2}$ has two possible channels:

$$
\begin{aligned}
\mathrm{SO}_{2}+h v & \rightarrow \mathrm{SO}+\mathrm{O}, \\
& \rightarrow \mathrm{S}+\mathrm{O}_{2},
\end{aligned}
$$

with quantum yields of $50 \%$ for each channel, as measured by Driscoll \& Warneck (1968) at $1849 \AA$. We adopt these quantum yields from the Lyman cutoff to the photodissociation threshold of channel (A.20), lying at $2070 \AA$ (Huebner et al. 1992), and assume that only channel (A.19) occurs from 2070 to $2179 \AA$, this latter value being the photodissociation threshold of the channel leading to SO (Huebner et al. 1992).

\section{A.28. $H F$}

Hydrogen fluoride is ionized by photons with energies above $16 \mathrm{eV}$ and thus only photodissociation is considered here. The photodissociation cross section of HF was obtained from the theoretical calculations by Li et al. (2010), which are in good agreement with the experimental values measured in the wavelength range 1070-1450 $\AA$ by Nee et al. (1985). The photodissociation cross section of HF has the shape of a continuous band centered at $\sim 1230 \AA$, which corresponds to the $X^{1} \Sigma^{+}-a^{1} \Pi$ transition.

\section{A.29. $\mathrm{HCl}$}

The photoabsorption cross section of hydrogen chloride has been measured by Brion et al. (2005). The photoionization yield up to the ionization threshold of $\mathrm{HCl}$, at $972.5 \AA$, has been taken from the study of Daviel et al. (1984).

\section{A.30. Photoionization of atoms}

We have also taken into account the photoionization cross sections of those atoms which can be ionized by photons 
with wavelengths longer than $911.776 \AA$. Among the elements included in the chemical network those atoms are, in order of increasing photoionization threshold (taken from Lide 2009 and given in parentheses): $\mathrm{Cl}(956.11 \AA), \mathrm{C}(1101.07 \AA)$, $\mathrm{P}(1182.30 \AA), \mathrm{S}(1196.76 \AA)$, Si (1520.97 $\mathrm{\AA}), \mathrm{Fe}(1568.9 \AA)$, $\operatorname{Mg}(1621.51 \AA)$, and $\mathrm{Na}(2412.58 \AA)$. Data for chlorine have been taken from the measurements by Ruscic \& Berkowitz (1983). Cross sections for sulfur and sodium were taken from the TOPbase database, while for phosphorus, carbon, and silicon we adopted the analytic fits by Verner et al. (1996) ${ }^{19}$. For magnesium and iron we used the cross sections in the Leiden database.

\section{Appendix B: Photodissociation and photoionization rates}

The photodissociation and photoionization rate of a given species depends on the relevant cross section and the strength and spectral shape of the FUV radiation field. In protoplanetary disks there are two main sources of FUV radiation, the interstellar radiation field (ISRF) and the star, each one having a different strength, spectral shape, and illumination geometry. Therefore, the relative contribution of each field to the various photoprocesses can be quite different depending on the particular process (via the spectral shape of the cross section) and the position in the disk (via the exposure to each radiation field).

The effect of the different spectral shapes of interstellar and stellar radiation fields on the photoprocesses occurring in protoplanetary disks has been investigated by van Dishoeck et al. (2006). These authors approximated the stellar emission of T Tauri and Herbig Ae stars as blackbodies at temperatures of $4000 \mathrm{~K}$ and $10000 \mathrm{~K}$, respectively. One of the most dramatic effects found is that the $4000 \mathrm{~K}$ blackbody field is much less efficient in photodissociating and photoionizing molecules than the interstellar and $10000 \mathrm{~K}$ blackbody fields. The reason is that the $4000 \mathrm{~K}$ blackbody emits little at short wavelengths, where dissociation and ionization take place. A similar study using an updated set of cross sections has been recently carried out by Heays et al. (2017). Here we have adopted as proxies of the T Tauri and Herbig Ae/Be radiation fields the spectra of TW Hya and AB Aurigae described in Sect. 2.1.1 and shown in Fig. 1. As a consequence of the strong FUV excess and Ly $\alpha$ emission usually present in T Tauri stars, which is accounted for by the TW Hya spectrum but not by a blackbody at $4000 \mathrm{~K}$, the photodissociation and photoionization efficiency of the T Tauri radiation field is greatly enhanced with respect to the blackbody assumption.

Following the idea of van Dishoeck et al. (2006), here we are interested in evaluating the effect of the different spectral shapes of interstellar and stellar radiation fields (see, e.g., Fig. B.1) on the photodissociation and photoionization rates of assorted species. To that purpose we scaled the T Tauri and Herbig Ae/Be radiation fields to get the same energy density of the ISRF over the wavelength range 912-2400 $\AA$, which amounts to $1.07 \times 10^{-13} \mathrm{erg} \mathrm{cm}^{-3}$ adopting the ISRF of Draine (1978) and the expressions given in Sect. 2.1.1. This is almost twice the value given by Habing (1968), $5.6 \times 10^{-14} \mathrm{erg} \mathrm{cm}^{-3}$. For a given radiation field characterized by a specific intensity $I_{\lambda}$ and a photoprocess characterized by a cross section $\sigma_{\lambda}$, the rate $\Gamma$ can be computed as

$\Gamma=\int_{912}^{\lambda_{\max }}\left(4 \pi \frac{\lambda}{h c} I_{\lambda}\right) \sigma_{\lambda} d \lambda$,

19 http://www . pa.uky.edu/ verner/photo.html

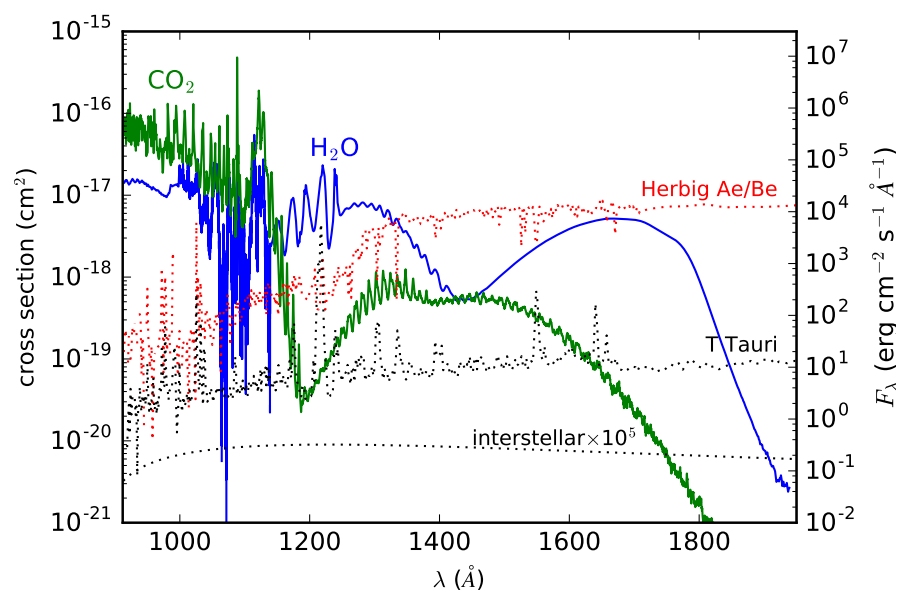

Fig. B.1. FUV photodissociation cross sections of $\mathrm{H}_{2} \mathrm{O}$ and $\mathrm{CO}_{2}$ superimposed on the FUV spectrum of the T Tauri and Herbig Ae/Be stars (flux at $1 \mathrm{au}$ ) and on the spectral shape of the interstellar radiation field. We note for example that $\operatorname{Ly} \alpha$ radiation becomes very important to photodissociate $\mathrm{H}_{2} \mathrm{O}$ but not $\mathrm{CO}_{2}$, which is photodissociated more efficiently at wavelengths shorter than $\sim 1150 \AA$.

where the term in parentheses is the photon flux per unit time, area, and wavelength, and the integral extends from the Lyman cutoff $(912 \AA)$ to a maximum wavelength $\lambda_{\max }$, which depends on each process.

In order to evaluate how the rates of the various photoprocesses vary with the depth into the disk, we made use of the Meudon PDR code (Le Petit et al. 2006) to compute the photodissociation and photoionization rates as a function of $A_{V}$. We consider a plane-parallel cloud illuminated on one side by an external radiation field corresponding to either the ISRF, the T Tauri star, or the Herbig Ae/Be star, where the stellar fields have been scaled to the FUV energy density of the ISRF. The cloud has uniform density of $\mathrm{H}$ nuclei and gas kinetic temperature. We verified that densities in the range $10^{4}-10^{8} \mathrm{~cm}^{-3}$ and temperatures ranging from 100 to $1000 \mathrm{~K}$, typical values in the photon dominated region of protoplanetary disks, yield identical results. The resulting photodissociation and photoionization rates as a function of the visual extinction have been fitted in the range $A_{V}=0-5$ according to the standard expression given by Eq. (7). We use this expression for simplicity, although we note that more accurate fits can be obtained with more elaborate expressions, for example, considering two coefficients instead of one in the exponential term (Roberge et al. 1991) or involving the 2nd-order exponential integral $E_{2}$ (Neufeld et al. 2009; Roueff et al. 2014; Heays et al. 2017).

In Table B.1 we list unattenuated rates $\alpha$ and attenuation factors $\gamma$ under different radiation fields for the photodissociation and photoionization processes involving the 29 molecules and 8 atoms discussed in Appendix $\mathrm{A}^{20}$. If we focus on the unattenuated rates $\alpha$, we see that the rates calculated under the T Tauri radiation field are not very different from those computed under the ISRF (within one order of magnitude), while in the case of

\footnotetext{
${ }^{20}$ The interstellar unattenuated rates $\alpha$ in Table B.1 have been scaled up by a factor of two with respect to the output values from the one-side illuminated clouds modeled with the Meudon PDR code. This way, our tabulated values are in line with those in the literature (Roberge et al. 1991; van Dishoeck et al. 2006; Heays et al. 2017) and in the astrochemical databases UMIST and KIDA. We note, however, that when modeling protoplanetary disks or clouds that are illuminated on just one side, the other being optically thick, one must use half the unattenuated rates $\alpha$ listed in Table B.1.
} 
A\&A 616, A19 (2018)

Table B.1. Photodissociation and photoionization rates for various radiation fields.

\begin{tabular}{|c|c|c|c|c|c|c|}
\hline \multirow[b]{2}{*}{ Reaction } & \multicolumn{2}{|c|}{ Interstellar $^{a}$} & \multicolumn{2}{|c|}{ T Tauri $^{b}$} & \multicolumn{2}{|c|}{ Herbig Ae/Be ${ }^{c}$} \\
\hline & $\alpha\left(\mathrm{s}^{-1}\right)$ & $\gamma$ & $\alpha\left(\mathrm{s}^{-1}\right)$ & $\gamma$ & $\alpha\left(\mathrm{s}^{-1}\right)$ & $\gamma$ \\
\hline $\mathrm{CH}+h v \rightarrow \mathrm{C}+\mathrm{H}$ & $9.8 \times 10^{-10}$ & 1.58 & $9.9 \times 10^{-10}$ & 1.02 & $2.5 \times 10^{-9}$ & 0.89 \\
\hline $\mathrm{CH}+h v \rightarrow \mathrm{CH}^{+}+\mathrm{e}^{-}$ & $9.3 \times 10^{-10}$ & 3.20 & $1.6 \times 10^{-10}$ & 2.71 & $1.1 \times 10^{-11}$ & 2.64 \\
\hline $\mathrm{CH}^{+}+h v \rightarrow \mathrm{C}+\mathrm{H}^{+}$ & $3.3 \times 10^{-10}$ & 3.02 & $1.0 \times 10^{-10}$ & 2.39 & $2.2 \times 10^{-11}$ & 1.89 \\
\hline $\mathrm{CH}_{2}+h v \rightarrow \mathrm{CH}+\mathrm{H}$ & $6.5 \times 10^{-10}$ & 1.89 & $3.3 \times 10^{-10}$ & 1.45 & $9.4 \times 10^{-10}$ & 1.42 \\
\hline $\mathrm{CH}_{3}+h v \rightarrow \mathrm{CH}_{2}+\mathrm{H}$ & $3.6 \times 10^{-10}$ & 2.06 & $2.0 \times 10^{-10}$ & 1.55 & $4.8 \times 10^{-10}$ & 1.54 \\
\hline $\mathrm{CH}_{3}+h v \rightarrow \mathrm{CH}+\mathrm{H}_{2}$ & $3.6 \times 10^{-10}$ & 2.06 & $2.0 \times 10^{-10}$ & 1.55 & $4.8 \times 10^{-10}$ & 1.54 \\
\hline $\mathrm{CH}_{3}+h v \rightarrow \mathrm{CH}_{3}^{+}+\mathrm{e}^{-}$ & $3.3 \times 10^{-10}$ & 2.91 & $5.4 \times 10^{-10}$ & 2.23 & $8.1 \times 10^{-12}$ & 2.32 \\
\hline $\mathrm{CH}_{4}+h v \rightarrow \mathrm{CH}_{3}+\mathrm{H}$ & $4.7 \times 10^{-10}$ & 2.75 & $7.8 \times 10^{-10}$ & 2.22 & $5.3 \times 10^{-11}$ & 2.07 \\
\hline $\mathrm{CH}_{4}+h v \rightarrow \mathrm{CH}_{2}+\mathrm{H}_{2}$ & $8.8 \times 10^{-10}$ & 2.83 & $1.0 \times 10^{-9}$ & 2.23 & $7.0 \times 10^{-11}$ & 2.09 \\
\hline $\mathrm{CH}_{4}+h v \rightarrow \mathrm{CH}+\mathrm{H}_{2}+\mathrm{H}$ & $1.2 \times 10^{-10}$ & 2.83 & $1.4 \times 10^{-10}$ & 2.23 & $9.7 \times 10^{-12}$ & 2.09 \\
\hline $\mathrm{CH}_{4}+h v \rightarrow \mathrm{CH}_{4}^{+}+\mathrm{e}^{-}$ & $1.4 \times 10^{-11}$ & 3.95 & $1.8 \times 10^{-12}$ & 3.36 & $1.4 \times 10^{-13}$ & 3.37 \\
\hline $\mathrm{C}_{2}+h v \rightarrow \mathrm{C}+\stackrel{4}{\mathrm{C}}$ & $1.3 \times 10^{-10}$ & 2.97 & $3.9 \times 10^{-11}$ & 2.52 & $3.4 \times 10^{-12}$ & 2.45 \\
\hline $\mathrm{C}_{2}+h v \rightarrow \mathrm{C}_{2}^{+}+\mathrm{e}^{-}$ & $2.1 \times 10^{-10}$ & 3.82 & $3.9 \times 10^{-11}$ & 3.23 & $2.3 \times 10^{-12}$ & 3.22 \\
\hline $\mathrm{C}_{2} \mathrm{H}+h v \rightarrow \mathrm{C}_{2}+\mathrm{H}$ & $1.6 \times 10^{-9}$ & 2.48 & $1.8 \times 10^{-9}$ & 2.13 & $5.6 \times 10^{-10}$ & 1.83 \\
\hline $\mathrm{C}_{2} \mathrm{H}_{2}+h v \rightarrow \mathrm{C}_{2} \mathrm{H}+\mathrm{H}$ & $4.4 \times 10^{-9}$ & 2.46 & $4.2 \times 10^{-9}$ & 2.08 & $1.9 \times 10^{-9}$ & 1.81 \\
\hline $\mathrm{C}_{2} \mathrm{H}_{2}+h v \rightarrow \mathrm{C}_{2} \mathrm{H}_{2}^{+}+\mathrm{e}^{-}$ & $3.3 \times 10^{-10}$ & 3.37 & $9.0 \times 10^{-11}$ & 2.87 & $6.0 \times 10^{-12}$ & 2.87 \\
\hline $\mathrm{OH}+h v \rightarrow \mathrm{O}+\mathrm{H}$ & $3.8 \times 10^{-10}$ & 2.33 & $5.1 \times 10^{-10}$ & 1.99 & $1.7 \times 10^{-10}$ & 1.62 \\
\hline $\mathrm{OH}+h v \rightarrow \mathrm{OH}^{+}+\mathrm{e}^{-}$ & $5.2 \times 10^{-12}$ & 3.95 & $6.5 \times 10^{-13}$ & 3.35 & $5.5 \times 10^{-14}$ & 3.37 \\
\hline $\mathrm{H}_{2} \mathrm{O}+h v \rightarrow \mathrm{OH}+\mathrm{H}$ & $6.8 \times 10^{-10}$ & 2.22 & $1.4 \times 10^{-9}$ & 2.01 & $3.4 \times 10^{-10}$ & 1.52 \\
\hline $\mathrm{H}_{2} \mathrm{O}+h v \rightarrow \mathrm{O}+\mathrm{H}_{2}$ & $1.0 \times 10^{-10}$ & 2.70 & $3.5 \times 10^{-10}$ & 2.21 & $9.5 \times 10^{-12}$ & 1.74 \\
\hline $\mathrm{H}_{2} \mathrm{O}+h v \rightarrow \mathrm{H}_{2} \mathrm{O}^{+}+\mathrm{e}^{-}$ & $2.7 \times 10^{-11}$ & 3.84 & $6.2 \times 10^{-12}$ & 3.22 & $3.6 \times 10^{-13}$ & 3.23 \\
\hline $\mathrm{O}_{2}+h v \rightarrow \mathrm{O}+\mathrm{O}$ & $7.3 \times 10^{-10}$ & 2.31 & $3.5 \times 10^{-10}$ & 1.81 & $5.6 \times 10^{-10}$ & 1.77 \\
\hline $\mathrm{O}_{2}+h v \rightarrow \mathrm{O}_{2}^{+}+\mathrm{e}^{-}$ & $5.1 \times 10^{-11}$ & 3.72 & $1.2 \times 10^{-11}$ & 3.08 & $8.6 \times 10^{-13}$ & 3.08 \\
\hline $\mathrm{H}_{2} \mathrm{CO}+h v \rightarrow \mathrm{CO}+\mathrm{H}_{2}$ & $1.6 \times 10^{-9}$ & 2.16 & $2.5 \times 10^{-9}$ & 1.80 & $1.0 \times 10^{-9}$ & 1.37 \\
\hline $\mathrm{H}_{2} \mathrm{CO}+h v \rightarrow \mathrm{H}_{2} \mathrm{CO}^{+}+\mathrm{e}^{-}$ & $4.3 \times 10^{-10}$ & 3.22 & $1.0 \times 10^{-10}$ & 2.76 & $7.6 \times 10^{-12}$ & 2.69 \\
\hline $\mathrm{CO}_{2}+h v \rightarrow \mathrm{CO}+\mathrm{O}$ & $1.1 \times 10^{-9}$ & 3.01 & $2.4 \times 10^{-10}$ & 2.41 & $4.3 \times 10^{-11}$ & 1.93 \\
\hline $\mathrm{NH}+h v \rightarrow \mathrm{N}+\mathrm{H}$ & $4.8 \times 10^{-10}$ & 2.46 & $2.0 \times 10^{-10}$ & 2.00 & $2.7 \times 10^{-10}$ & 1.94 \\
\hline $\mathrm{NH}+h v \rightarrow \mathrm{NH}^{+}+\mathrm{e}^{-}$ & $1.8 \times 10^{-12}$ & 4.00 & $7.4 \times 10^{-15}$ & 3.41 & $1.7 \times 10^{-14}$ & 3.42 \\
\hline $\mathrm{NH}_{2}+h v \rightarrow \mathrm{NH}+\mathrm{H}$ & $8.9 \times 10^{-10}$ & 1.92 & $4.2 \times 10^{-10}$ & 1.50 & $1.1 \times 10^{-9}$ & 1.47 \\
\hline $\mathrm{NH}_{2}+h v \rightarrow \mathrm{NH}_{2}^{+}+\mathrm{e}^{-}$ & $1.1 \times 10^{-10}$ & 3.44 & $3.1 \times 10^{-11}$ & 2.92 & $2.1 \times 10^{-12}$ & 2.91 \\
\hline $\mathrm{NH}_{3}+h v \rightarrow \mathrm{NH}_{2}+\mathrm{H}$ & $1.2 \times 10^{-9}$ & 1.99 & $6.9 \times 10^{-10}$ & 1.58 & $1.2 \times 10^{-9}$ & 1.41 \\
\hline $\mathrm{NH}_{3}+h v \rightarrow \mathrm{NH}+\mathrm{H}_{2}$ & $3.1 \times 10^{-10}$ & 2.72 & $1.1 \times 10^{-9}$ & 2.21 & $3.6 \times 10^{-11}$ & 2.03 \\
\hline $\mathrm{NH}_{3}+h v \rightarrow \mathrm{NH}_{3}^{+}+\mathrm{e}^{-}$ & $4.2 \times 10^{-10}$ & 3.04 & $1.8 \times 10^{-10}$ & 2.34 & $8.2 \times 10^{-12}$ & 2.50 \\
\hline $\mathrm{N}_{2}+h v \rightarrow \mathrm{N}+\mathrm{N}$ & $3.6 \times 10^{-10}$ & 3.81 & $7.6 \times 10^{-11}$ & 3.21 & $3.9 \times 10^{-12}$ & 3.19 \\
\hline $\mathrm{CN}+h v \rightarrow \mathrm{C}+\mathrm{N}$ & $1.0 \times 10^{-9}$ & 3.55 & $1.8 \times 10^{-10}$ & 3.02 & $1.2 \times 10^{-11}$ & 3.04 \\
\hline $\mathrm{HCN}+h v \rightarrow \mathrm{CN}+\mathrm{H}$ & $1.9 \times 10^{-9}$ & 2.82 & $4.5 \times 10^{-9}$ & 2.22 & $1.6 \times 10^{-10}$ & 2.01 \\
\hline $\mathrm{HNC}+h v \rightarrow \mathrm{CN}+\mathrm{H}$ & $9.4 \times 10^{-10}$ & 2.45 & $3.2 \times 10^{-9}$ & 2.16 & $3.9 \times 10^{-10}$ & 1.80 \\
\hline $\mathrm{NO}+h v \rightarrow \mathrm{N}+\mathrm{O}$ & $4.7 \times 10^{-10}$ & 2.01 & $2.3 \times 10^{-10}$ & 1.60 & $4.1 \times 10^{-10}$ & 1.41 \\
\hline $\mathrm{NO}+h v \rightarrow \mathrm{NO}^{+}+\mathrm{e}^{-}$ & $2.6 \times 10^{-10}$ & 3.00 & $2.4 \times 10^{-10}$ & 2.27 & $8.7 \times 10^{-12}$ & 2.24 \\
\hline $\mathrm{SH}+h v \rightarrow \mathrm{S}+\mathrm{H}$ & $1.3 \times 10^{-9}$ & 1.89 & $2.0 \times 10^{-9}$ & 1.54 & $1.8 \times 10^{-9}$ & 1.30 \\
\hline $\mathrm{SH}^{+}+h v \rightarrow \mathrm{S}+\mathrm{H}^{+}$ & $6.9 \times 10^{-10}$ & 1.89 & $3.2 \times 10^{-10}$ & 1.05 & $3.9 \times 10^{-10}$ & 0.80 \\
\hline $\mathrm{H}_{2} \mathrm{~S}+h v \rightarrow \mathrm{SH}+\mathrm{H}$ & $3.2 \times 10^{-9}$ & 2.26 & $3.4 \times 10^{-9}$ & 1.91 & $1.8 \times 10^{-9}$ & 1.58 \\
\hline $\mathrm{H}_{2} \mathrm{~S}+h v \rightarrow \mathrm{H}_{2} \mathrm{~S}^{+}+\mathrm{e}^{-}$ & $7.2 \times 10^{-10}$ & 3.15 & $1.7 \times 10^{-10}$ & 2.68 & $1.3 \times 10^{-11}$ & 2.61 \\
\hline $\mathrm{CS}+h v \rightarrow \mathrm{C}+\mathrm{S}$ & $9.5 \times 10^{-10}$ & 2.60 & $4.2 \times 10^{-9}$ & 2.20 & $1.7 \times 10^{-10}$ & 1.98 \\
\hline $\mathrm{CS}+h v \rightarrow \mathrm{CS}^{+}+\mathrm{e}^{-}$ & $1.7 \times 10^{-10}$ & 3.30 & $4.1 \times 10^{-11}$ & 2.81 & $2.8 \times 10^{-12}$ & 2.81 \\
\hline $\mathrm{SO}+h v \rightarrow \mathrm{S}+\mathrm{O}$ & $4.8 \times 10^{-9}$ & 2.24 & $1.1 \times 10^{-8}$ & 2.01 & $2.1 \times 10^{-9}$ & 1.47 \\
\hline $\mathrm{SO}+h v \rightarrow \mathrm{SO}^{+}+\mathrm{e}^{-}$ & $2.1 \times 10^{-10}$ & 3.18 & $5.3 \times 10^{-11}$ & 2.72 & $4.0 \times 10^{-12}$ & 2.64 \\
\hline $\mathrm{SO}_{2}+h v \rightarrow \mathrm{SO}+\mathrm{O}$ & $1.2 \times 10^{-9}$ & 2.25 & $2.1 \times 10^{-9}$ & 1.97 & $5.5 \times 10^{-10}$ & 1.47 \\
\hline $\mathrm{SO}_{2}+h v \rightarrow \mathrm{S}+\mathrm{O}_{2}$ & $1.1 \times 10^{-9}$ & 2.29 & $2.1 \times 10^{-9}$ & 2.02 & $4.1 \times 10^{-10}$ & 1.47 \\
\hline $\mathrm{SO}_{2}+h v \rightarrow \mathrm{SO}_{2}^{+}+\mathrm{e}^{-}$ & $1.3 \times 10^{-10}$ & 3.77 & $3.0 \times 10^{-11}$ & 3.17 & $2.1 \times 10^{-12}$ & 3.16 \\
\hline $\mathrm{HF}+h v \rightarrow \mathrm{H}+\mathrm{F}$ & $1.3 \times 10^{-10}$ & 2.57 & $3.1 \times 10^{-10}$ & 2.18 & $2.7 \times 10^{-11}$ & 1.84 \\
\hline $\mathrm{HCl}+h v \rightarrow \mathrm{H}+\mathrm{Cl}$ & $2.0 \times 10^{-9}$ & 2.53 & $7.7 \times 10^{-10}$ & 1.99 & $4.2 \times 10^{-10}$ & 1.68 \\
\hline $\mathrm{HCl}+h v \rightarrow \mathrm{HCl}^{+}+\mathrm{e}^{-}$ & $1.9 \times 10^{-11}$ & 3.91 & $2.7 \times 10^{-12}$ & 3.33 & $1.7 \times 10^{-13}$ & 3.35 \\
\hline $\mathrm{C}+h v \rightarrow \mathrm{C}^{+}+\mathrm{e}^{-}$ & $3.3 \times 10^{-10}$ & 3.28 & $8.4 \times 10^{-11}$ & 2.82 & $5.8 \times 10^{-12}$ & 2.80 \\
\hline $\mathrm{Si}+h v \rightarrow \mathrm{Si}^{+}+\mathrm{e}^{-}$ & $4.2 \times 10^{-9}$ & 2.49 & $4.4 \times 10^{-9}$ & 2.13 & $2.1 \times 10^{-9}$ & 1.91 \\
\hline $\mathrm{P}+h v \rightarrow \mathrm{P}^{+}+\mathrm{e}^{-}$ & $1.1 \times 10^{-9}$ & 2.99 & $2.5 \times 10^{-10}$ & 2.48 & $2.3 \times 10^{-11}$ & 2.45 \\
\hline $\mathrm{S}+h v \rightarrow \mathrm{S}^{+}+\mathrm{e}^{-}$ & $9.7 \times 10^{-10}$ & 3.09 & $2.1 \times 10^{-10}$ & 2.62 & $1.8 \times 10^{-11}$ & 2.55 \\
\hline $\mathrm{Cl}+h v \rightarrow \mathrm{Cl}^{+}+\mathrm{e}^{-}$ & $4.7 \times 10^{-11}$ & 3.94 & $6.1 \times 10^{-12}$ & 3.35 & $4.4 \times 10^{-13}$ & 3.36 \\
\hline $\mathrm{Na}+h v \rightarrow \mathrm{Na}^{+}+\mathrm{e}^{-}$ & $1.3 \times 10^{-11}$ & 2.12 & $1.6 \times 10^{-11}$ & 1.68 & $8.4 \times 10^{-12}$ & 1.47 \\
\hline $\mathrm{Mg}+h v \rightarrow \mathrm{Mg}^{+}+\mathrm{e}^{-}$ & $6.7 \times 10^{-11}$ & 2.29 & $4.4 \times 10^{-11}$ & 1.80 & $5.4 \times 10^{-11}$ & 1.73 \\
\hline $\mathrm{Fe}+h v \rightarrow \mathrm{Fe}^{+}+\mathrm{e}^{-}$ & $4.7 \times 10^{-10}$ & 2.45 & $6.9 \times 10^{-10}$ & 2.02 & $2.8 \times 10^{-10}$ & 1.88 \\
\hline
\end{tabular}

Notes. ${ }^{(a)}$ Interstellar radiation field (ISRF) of Draine (1978); see expressions in Sect. 2.1.1. ${ }^{(b)}$ Radiation field of TW Hya scaled to the FUV energy density of the ISRF. ${ }^{(c)}$ Radiation field of AB Aurigae scaled to the FUV energy density of the ISRF. 
the Herbig Ae/Be radiation field some photoprocesses have rates similar to those computed under the ISRF (within one order of magnitude) while for others the rates are lower by more than a factor of ten. By looking to the spectral shape of the different FUV radiation fields (see Fig. B.1) we note that, except for the fact that stellar spectra have spectral features while the ISRF is a continuum, the spectra of the T Tauri star and the ISRF are more flat than that of the Herbig Ae/Be star, which shows a depletion of flux at short wavelengths (below $\sim 1300 \AA$ ). Therefore, those photoprocesses which occur more effectively at short wavelengths, as, for example, the photodissociation of $\mathrm{N}_{2}$ (see Fig.A.1), have higher rates under the ISRF and the T Tauri radiation field than under the Herbig Ae/Be field.

It is interesting to compare our results with previous studies. In general, the unattenuated rates $\alpha$ and the attenuation parameters $\gamma$ calculated here under the ISRF are similar to those calculated by Roberge et al. (1991), van Dishoeck et al. (2006), and Heays et al. (2017). As concerns the rates under the Herbig
Ae/Be radiation field, our parameters $\alpha$ and $\gamma$ are also not very different from those calculated by van Dishoeck et al. (2006) and Heays et al. (2017) for a $10000 \mathrm{~K}$ blackbody radiation field, because the FUV spectrum of AB Aurigae used by us is not drastically different from that of a $10000 \mathrm{~K}$ blackbody. The same is not true in the case of the radiation field of the T Tauri star. The FUV spectrum of a $4000 \mathrm{~K}$ blackbody is very different from that of TW Hya, which translates into very different photodissociation and photoionization rates. In general, the rates calculated by us for a T Tauri star are orders of magnitude higher than those computed by van Dishoeck et al. (2006) and Heays et al. (2017).

The values provided in Table B.1 can be useful in chemical models of protoplananetary disks around T Tauri and Herbig Ae/Be stars, as long as they allow for computation of the photodissociation and photoionization rates in a simple way, avoiding the more expensive approach, in terms of computing time, of solving the FUV radiative transfer. 